Göttingen Series

in Biophysics

\title{
Johanna Block
}

\section{Stress-Strain Behavior of Single Vimentin Intermediate Filaments}
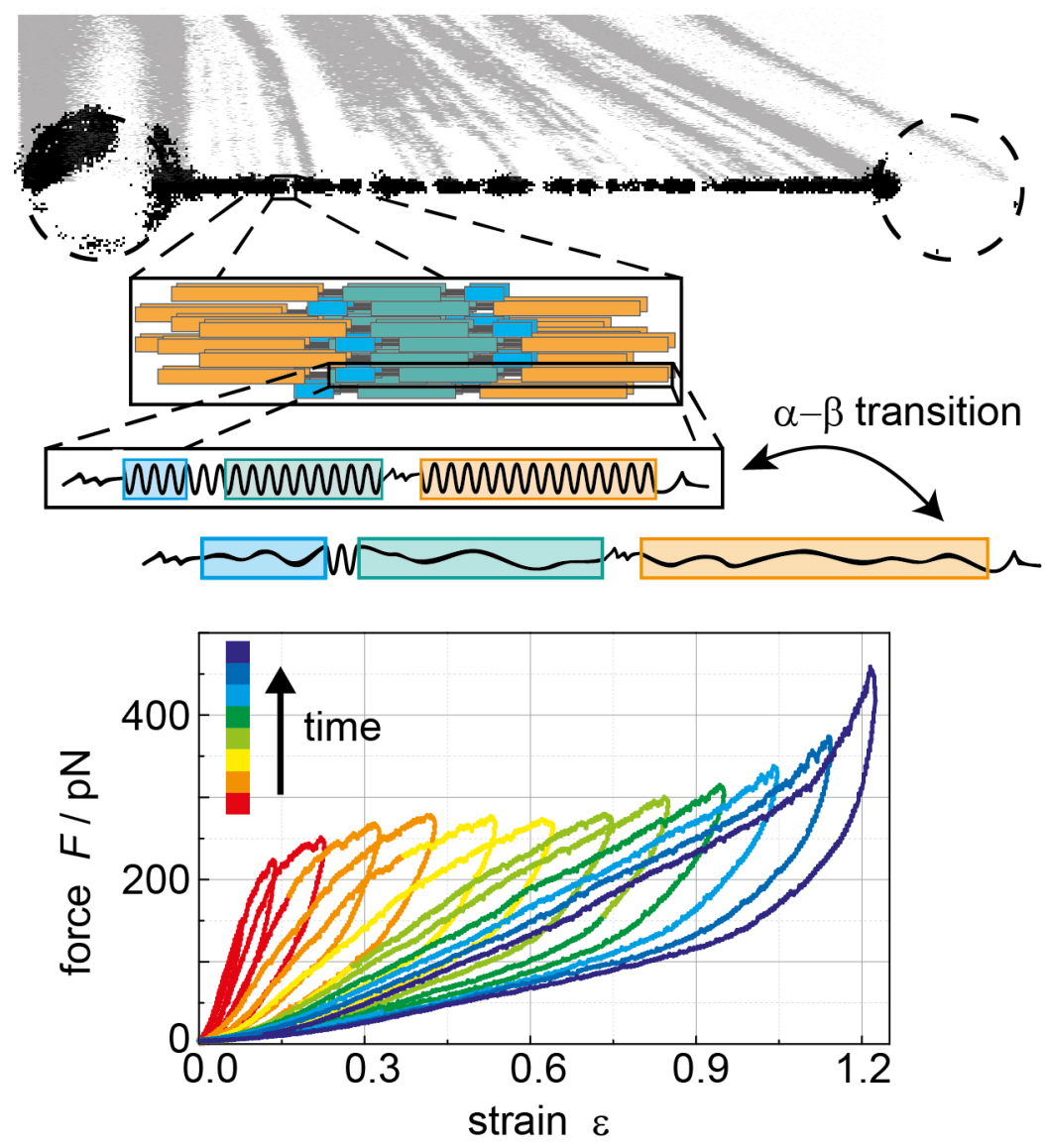

Johanna Lena Block

Stress-Strain Behavior of Single Vimentin Intermediate Filament

This work is licensed under a

Attribution-NonCommercial 4.0 International

(CC BY-NC 4.0)

(). $(1) \Theta$ 
Published in 2018 by Universitätsverlag Göttingen

as volume 1 in the series "Göttingen Series in Biophysics" 
Johanna Lena Block

Stress-Strain Behavior of Single Vimentin Intermediate Filament

Göttingen Series in Biophysics Volume 1

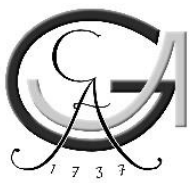

Universitätsverlag Göttingen 2018 
Bibliographische Information der Deutschen Nationalbibliothek

The Deutsche Nationalbibliothek lists this publication in the Deutsche Nationalbibliografie; detailed bibliographic data are available on the Internet at http://dnb.dnb.de.

This work was supported by the DFG Collaborative Research Centers SFB 755

"Nanoscale Photonic Imaging" (project b7) and the ERC under the European Union's Horizon 2020 research and innovation programme (Consolidator Grant, grant agreement no. 724932 and Laserlab Europe, grant no. 654148

Address of the author

Johanna Lena Block

Email: johanna-block@gmx.net; johanna.block@phys.uni-goettingen.de

Dissertation for the award of the degree "Doctor rerum naturalium" of the Georg-August-Universität Göttingen within the doctoral program

Göttingen Graduate School for Neurosciences, Biophysics, and Molecular Biosciences (GGNB) of the Georg-August University School of Science (GAUSS)

Members of the thesis committee:

Prof. Dr. Sarah Köster (Reviewer)

Prof. Dr. Andreas Janshoff (Reviewer)

Dr. Florian Rehfeldt

This work is protected by German Intellectual Property Right Law.

It is also available as an Open Access version through the publisher's homepage and the Göttingen University Catalogue (GUK) at the Göttingen State and University Library (http://www.sub.uni-goettingen.de). The license terms of the online version apply.

Set and layout: Johanna Block

Cover: Jutta Pabst

Cover image: Johanna Block

(C) 2018 Universitätsverlag Göttingen

https://univerlag.uni-goettingen.de

ISBN: 978-3-86395-373-7

DOI: https://doi.org/10.17875/gup2018-1102

e-ISSN: 2626-207X 


\section{Preface of the series editors}

The Göttingen Series in Biophysics is intended as a collection of research monographs in biological physics, carried out at the Institute for X-Ray Physics at the University of Göttingen, and in the framework of related research networks and collaborations. The series covers topics from molecular to cellular biophysics, includes experimental, numerical then theoretical work and combines methods development and studies of complex biological systems.

In most cases, the contributions are based on $\mathrm{PhD}$ theses. With this book series, we intend to promote the individual monographs by putting them into the larger context of the research field. We hope to be able to enhance the visibility of the research carried out here, and to encourage others to build on our findings, use our methods and advance the filed in a collaborative spirit.

Prof. Dr. Sarah Köster

Prof. Dr. Tim Salditt

Editors

Göttingen, August 2018 



\section{Contents}

1 Introduction 1

References . . . . . . . . . . . . . . . . . . . . 4

2 State of the Art 5

2.1 Intermediate Filaments . . . . . . . . . . . . . . . . . 5

2.1.1 Architecture of Intermediate Filaments . . . . . . . . . . 6

2.1.2 Assembly of Intermediate Filaments . . . . . . . . . . . 7

2.1.3 Properties of Intermediate Filaments - Similar, but not Identical 9

2.2 Intermediate Filaments under Load . . . . . . . . . . . . . 10

2.2.1 Mechanics of Individual Intermediate Filaments ～～～～～～. . . 11

2.2.2 Simulations of Intermediate Filament Mechanics . . . . . . 13

2.2.3 Intermediate Filaments and the Mechanical Properties of Cells. . 14

2.3 Mathematical Models for Biopolymers . . . . . . . . . . . . . . 17

2.3.1 Freely-jointed Chain and Worm-like Chain . . . . . . . . 17

2.3.2 Entropic Springs . . . . . . . . . . . . . . . . . 21

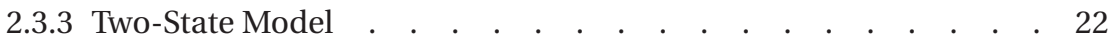

2.4 Optical Tweezers. . . . . . . . . . . . . . . . . . . . 24

2.4.1 Physics of Optical Trapping . . . . . . . . . . . . . . . . . $\quad$. 25

2.4.2 Calibration of Optical Traps . . . . . . . . . . . . . . . . . $\quad$. 27

References . . . . . . . . . . . . . . . . . . . . . . . 31

$3 \quad$ Materials and Methods $\quad 41$

3.1 Vimentin expression and purification $\quad$. . . . . . . . . . . . . . . 41 


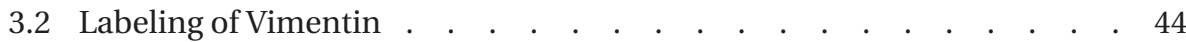

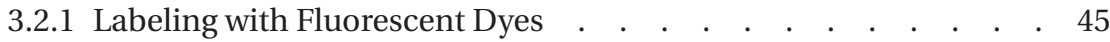

3.2.2 Labeling with Biotin . . . . . . . . . . . . . . . . 46

3.3 Vimentin Reconstitution and Assembly . . . . . . . . . . . . . . 47

3.4 Maleimide-functionalization of Beads . . . . . . . . . . . . . . . . $\quad$. 48

3.5 Optical Tweezers Experiments . . . . . . . . . . . . . . . 48

3.5.1 Setup . . . . . . . . . . . . . . . . . . . . . . 49

3.5.2 Calibration of Optical Traps . . . . . . . . . . . . . . . . . . 49

3.5.3 Sample Preparation . . . . . . . . . . . . . . . . 50

3.5.4 Measurement Preparation and Basic Experimental Procedure . . 51

3.5.5 Measuring Procedures . . . . . . . . . . . . . . . 52

3.5.6 Cleaning Protocol for the Flow Cell . . . . . . . . . . . . 55

3.6 Data Processing . . . . . . . . . . . . . . . . . . . . 56

3.6.1 Processing for further analysis . . . . . . . . . . . . . . . 58

3.6.2 Processing Force-Distance Curves . . . . . . . . . . . . . . 59

3.6.3 Processing of Force Clamp and Creep Data . . . . . . . . . . 60

3.6.4 Correction of Force Offset . . . . . . . . . . . . . . . . . . . 60

References . . . . . . . . . . . . . . . . . . 64

4 Nonlinear Loading-Rate- Dependent Force Response of Individual Vimentin Intermediate Filaments to Applied Strain 65

4.1 Abstract . . . . . . . . . . . . . . . . . . . . . . 67

4.2 Introduction . . . . . . . . . . . . . . . . . . . . . 67

4.3 Materials and Methods $\quad . \quad$. . . . . . . . . . . . . . . . . . . . . 69

4.4 Results . . . . . . . . . . . . . . . . . . . . . . . 70

4.5 Discussion. . . . . . . . . . . . . . . . . . . . . . 75

4.6 Supplementary Information . . . . . . . . . . . . . . . . 78

4.6.1 Theoretical model . . . . . . . . . . . . . . . . . . . . 78

4.6.2 Comparison of Worm Like and Freely Jointed Chains . . . . . 79

4.6.3 Fit Results. . . . . . . . . . . . . . . . . . . . 80

References . . . . . . . . . . . . . . . . . . . . . . 83 
5 Viscoelastic Properties of Vimentin Originate from Nonequilibrium Con$\begin{array}{lr}\text { formational Changes } & 87\end{array}$

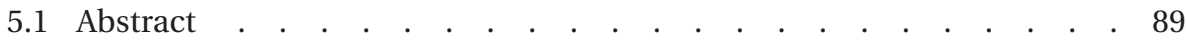

5.2 Introduction . . . . . . . . . . . . . . . . . . . . . . . . . . 89

5.3 Results . . . . . . . . . . . . . . . . . . . . . . . 91

5.3.1 Pronounced Energy Dissipation . . . . . . . . . . . . . 91

5.3.2 A Biopolymer with Tensile Memory . . . . . . . . . . . . . 993

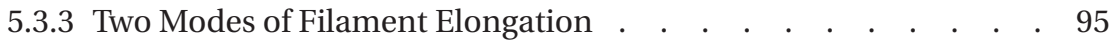

5.4 Discussion. . . . . . . . . . . . . . . . . . . . . . . . . 97

5.5 Material and Methods . . . . . . . . . . . . . . . . . . . . . . . 99

5.5.1 Vimentin Expression and Purification Protocol . . . . . . . . 99

5.5.2 Vimentin Labeling . . . . . . . . . . . . . . . . . 102

5.5.3 Vimentin Reconstruction and Assembly . . . . . . . . . . 102

5.5.4 Maleimide Functionalization of Beads . . . . . . . . . . 103

5.5.5 Experimental setup and procedure . . . . . . . . . . . 103

5.5.6 Analysis of Optical Tweezers Data. . . . . . . . . . . . 104

5.6 Models . . . . . . . . . . . . . . . . . . . . . . . 105

5.6.1 Effective Two-State Model . . . . . . . . . . . . . . . 105

5.6.2 Analytical Solution for FC Conditions . . . . . . . . . . . . 108

5.6.3 MC Simulation of Unfolding and Refolding of Parallel Elements 108

5.7 Supplementary Materials . . . . . . . . . . . . . . . . . 112

5.7.1 Movie S1 . . . . . . . . . . . . . . . . . . 117

References . . . . . . . . . . . . . . . . . . . . . . . 120

6 Mechanical Response of Glutaraldehyde Stabilized Vimentin IFs 125

6.1 Introduction . . . . . . . . . . . . . . . . . . . . . 125

6.2 Loading-Rate-Dependency . . . . . . . . . . . . . . . . 128

6.3 Creep Behavior at Constant Force . . . . . . . . . . . . . . . 130

6.4 Discussion. . . . . . . . . . . . . . . . . . . . . . 131

References . . . . . . . . . . . . . . . . . . . . . . . 134

$7 \quad$ Summary, Discussion and Conclusion 137

References . . . . . . . . . . . . . . . . . . . . . . 143 
List of Publications

Lebenslauf

Danksagung

A Supplementary Information - Energy Dissipation 


\section{List of Figures}

1.1 Fluorescence Images of the Cytoskeletal Proteins . . . . . . . . . . . . 2

2.1 Vimentin Assembly Scheme . . . . . . . . . . . . . . . 8

2.3 Results of simulated stretching events of vimentin dimers and tetramers 15

2.4 FJC and WLC . . . . . . . . . . . . . . . . . . . 17

2.5 FJC, scalar product an representation of an elastic filament with a constant curvature .......................... 19

2.6 Sketch of a double well potential . . . . . . . . . . . . . . . . . . 23

2.7 Ray Optics in a highly focused laser beam. . . . . . . . . . . . . . . 25

3.1 Reaction scheme of the maleimide cysteine coupling . . . . . . . . . . . 44

3.2 Excitation and emission spectrum of ATTO647N $\ldots \ldots \ldots \ldots$

3.3 Illustration of a dual optical trap $\ldots \ldots \ldots \ldots \ldots$

3.4 Experimental setup and flow cell $\ldots \ldots \ldots \ldots \ldots \ldots$

3.5 Experimental Steps Diagram $\ldots \ldots \ldots \ldots \ldots$ 
3.6 Fluorescence microscopy examples . . . . . . . . . . . . . . . 53

3.7 Example curves of measuring procedures . . . . . . . . . . . . . . . 57

4.1 Schematic representation of vimentin filament formation and Overview

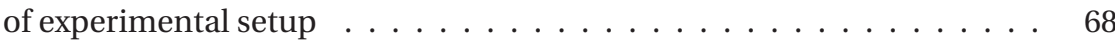

4.2 Force-strain curves recorded with the OT at different loading rates . . . . 71

4.3 Sketch of two-state eFJC model and example fit . . . . . . . . . . . . . 72

4.4 Comparison of AFM and OT experiments . . . . . . . . . . . . . . . 74

4.5 Comparison of WLC (black) and eFJC (red) models. . . . . . . . . . . . . . 80

4.6 Fit results of $k_{\text {eff. }}$ The black line shows the average value of the distribution. 81

4.7 a) Fit results of $x_{\mathrm{u}, 1}$, b) $x_{\mathrm{u}, 2}$, c) $x_{\mathrm{u}, 3}$, with the average values of the distributions (black lines) . . . . . . . . . . . . . . . . . . . . . . . . . 82

5.1 Experimental setup, vimentin assembly and comparison of effective twostate model and experimental data. . . . . . . . . . . . . . . . . . . . 91

5.2 Response to repeated strain. . . . . . . . . . . . . . . . . . . 94

5.3 Response of individual vimentin IFs to applied constant force. . . . . . . 96

5.4 Vimentin Assembly: IF formation follows a complex and hierarchical scheme. . . . . . . . . . . . . . . . . . . . . . . . . . 112

5.5 Comparison of calculated (two-state-model) stretching and relaxation curves for different values of the persistence lengths $L_{\mathrm{P}} \ldots \ldots \ldots$. . . . . . 113

5.6 Sketch of the equivalent circuit diagram used for setting up the MC simulations. . . . . . . . . . . . . . . . . . . . . . . . . . . . . 114

5.7 Step size analysis of force clamp data sets. . . . . . . . . . . . . . . . . . 114 
5.8 Step size analysis of data sets with filaments covalently bond to beads via malemide-chemistry. . . . . . . . . . . . . . . . 115

5.9 Step size analysis of relaxation data sets. . . . . . . . . . . . . . . . 115

5.10 Vimentin relaxation experiments. . . . . . . . . . . . . . 116

5.11 Data corresponding to the cycle shown in movie $\mathrm{S} 1 . \ldots \ldots \ldots$

5.12 Quality control of labeled, assembled vimentin filaments. . . . . . . . . . 118

5.13 Simulations, cycles, and comparison for different numbers of monomers per ULF . . . . . . . . . . . . . . . . . . . . . . . . . . . 119

6.1 General monomer and dimer structure and different types of tetramers of vimentin IFs. . . . . . . . . . . . . . . . . . . . . 126

6.2 Velocity dependent behavior of untreated and glutaraldehyde stabilized vimentin filaments. . . . . . . . . . . . . . . . . . . . . 128

6.3 Velocity dependent behavior of untreated and glutaraldehyde stabilized vimentin filaments - full stretching and relaxation cycle. . . . . . . . . . . 130

6.4 Relative energy dissipation in glutaraldehyde stabilized and untreated filaments. . . . . . . . . . . . . . . . . . . 131

6.5 Force clamps of glutaraldehyde stabilized and non-stabilized vimentin IFs. 132

6.6 Step size histograms of glutaraldehyde stabilized vimentin filaments. . . 133

A.1 Schematic representation of areas integrated to calculate energy dissipation from full stretching-relaxation data sets . . . . . . . . . . . . . . . 154

A.2 Histograms of the amount of introduced energy. . . . . . . . . . . 155

A.3 Histograms of the amount of dissipated energy. . . . . . . . . . . . . . . 156 
A.4 Average and standard deviation of introduced and dissipated energy for glutaraldehyde stabilized and untreated vimentin filaments. . . . . . . . 157

A.5 Introduced, dissipated and relative dissipated energy in glutaraldehyde stabilized and untreated vimentin filaments binned by the maximum reached strain. . . . . . . . . . . . . . . . . . . . . . . . . . . . . 158 


\section{List of Tables}

2.1 Sequence homology classes of intermediate filament proteins and their occurrence in mammals. Table according to and adopted from Ref. [5] . 6

3.1 List of Chemicals and Enzymes . . . . . . . . . . . . . . 61 



\section{Chapter 1}

\section{Introduction}

The human body consists of about 200 different cell types and an almost uncountable number of single cells [1]. All of these cells have to perform as accurate as possible to keep the formed organism viable and powerful. On the single cell level this means that all cells have to fulfill a plethora of functions that, among other demands, require self-organization in space [2]. They have to chemically and mechanically interact with each other and with their environment [3] and adapt to diverse conditions. They need to rearrange, e.g. during cell division or differentiation [4] and follow a complex construction plan when a new organism is formed.

Focusing on the mechanical challenges cells are exposed to, every single cell requires physical properties which are adapted to its specific environment [5]. By comparing the shape and size of cells which are as different as e.g. nerve cells, branched and connected by long, thin tubes, red blood cells, tiny and in the mature state lacking a nucleus, and fibroblasts, loosely connected, highly motile cells, one can get an impression of the diversity cells developed yet within one organism [6]. However, the underlying architecture of all these shapes is the cytoskeleton, a remarkable and complex system that is widely accepted to play a major role also for the mechanical strength of cells [2]. The three main filamentous building blocks of the cytoskeleton, microtubules (MTs), microfilaments or actin filaments (MFs) and the protein family of intermediate filaments (IFs) [2] are shown fluorescently stained in Fig. 1.1. 

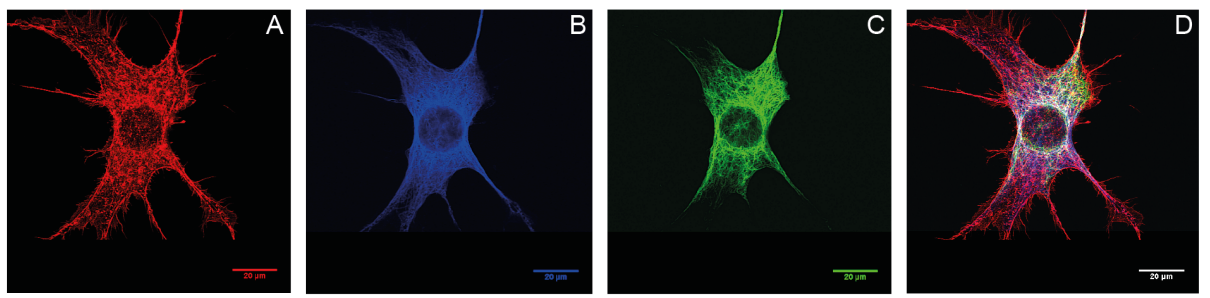

Figure 1.1: Fluorescence images of the cytoskeletal proteins actin or microfilaments (A), microtubules (B) and vimentin (intermediate filaments) (C) of a 3 T3 fibroblast cell. (D) shows the overlay of all channels. Images courtesy of Dr. Ulrike Rölleke.

Each of the three filament types has specific mechanical properties and fulfills its unique role as a member of the cytoskeleton. To do so, hundreds of accessory, regulating and interlinking proteins are involved. Three actin isoforms are known in vertebrates. While only muscle cells express $\alpha$-actin, almost all other cells express $\beta$ - and $\gamma$-actin. Actin participates in the maintenance of the cell shape as well as in cell division and signalling [2]. It is also important for muscle contraction, force generation and cell migration [2]. MTs are important, e.g., for the correct positioning of organelles and serve as a basis for directed intracellular transport $[2,5]$. They have also a prominent role in cell division where they form the mitotic spindle, a structure that separates the chromosomes [2]. Within the cytoskeletal proteins, IFs are the least well studied ones, however, they are believed to support the cell with mechanical resistance [2, 7]. A detailed overview about the features and properties of the members of the cytoskeleton can be found, e.g., in reference [2]. A more detailed review on the properties and functions of IFs is given in Section 2.1 and 2.2.

In the context of this thesis, the response of individual vimentin IFs to applied strain was directly probed in order to gain knowledge about their mechanical properties on the single filament level. Using a combination of optical tweezers measurements, confocal fluorescent microscopy and microfluidics the mechanical and physical properties of individual vimentin filaments were measured in a highly controlled fashion and depending on e.g. loading rate [8] or stretching history. From the experimental data and theoretical modeling, as well as Monte Carlo simulations, it was possible to particularly link the mechanical properties to the molecular structure and hierarchical ar- 
chitecture of IFs. The underlying concepts of conformational changes due to applied strain and energy dissipation allow for speculations on the impact of IFs for cellular integrity and resistance especially in situations of high stresses.

Chapter 2 provides an overview of the role of IFs by focusing on the hierarchical architecture and assembly process. Some major differences between IFs and the other two cytoskeletal filamentous proteins are pointed out. Section 2.2 presents a review on IF mechanics on the single filament level as well as IFs in the context of cells and IFs in computer simulations and thereby embeds the performed experiments into the existing literature. The second part of Chapter 2 deals with the background of the physical models that were used to fit and simulate the experimental data. In the last part of Chapter 2 an introduction to optical tweezers (OTs) and a brief description of the physics behind them is given. The chapter is closed by an overview on how the calibration of the force readout of OTs can be performed.

A detailed explanation of the preparation of experiments, the experimental procedures, the production, purification, labeling and assembly of vimentin and a short description of the experimental setup are given in Chapter 3.

Chapter 4 is a reprint of the results which are published as "Nonlinear LoadingRate-Dependent Force Response of Individual Vimentin Intermediate Filaments to Applied Strain" [8], and the results in Chapter 5 are published under the title "Viscoelastic Properties of Vimentin Originate from Nonequilibrium Conformational Changes" [9]. Results obtained from glutaraldehyde stabilized vimentin IFs are presented and compared to non-stabilized filaments in Chapter 6.

Finally, in Chapter 7, the most important results are summarized and their impact on understanding the mechanics of single IFs are pointed out. Reasonable speculations on the physical functions of IFs as well as their role for the cell are presented and ideas for experiments are proposed to further investigate the suggested molecular mechanisms that were already observed in IF superstructures but not directly measured on the single filament level. 
Chapter 1. Introduction

\section{References}

1. Boal, D. H. Mechanics of the Cell Second edition (Cambridge University Press, Cambridge, 2012).

2. Alberts, B. et al. Molecular Biology of the Cell Sixth edition (Garland Science Taylor and Francis Group, New York, NY, 2015).

3. Herrmann, H., Bär, H., Kreplak, L., Strelkov, S. V. \& Aebi, U. Intermediate Filaments: From Cell Architecture to Nanomechanics. Nat. Rev. Mol. Cell Bio. 8, 562573 (2007).

4. Huber, F., Boire, A., López, M. P. \& Koenderink, G. H. Cytoskeletal Crosstalk: When Three Different Personalities Team Up. Curr. Opin. Cell Biol. 32C, 39-47 (2014).

5. Block, J., Schroeder, V., Pawelzyk, P., Willenbacher, N. \& Koster, S. Physical Properties of Cytoplasmic Intermediate Filaments. Biochim. Biophys. Acta 1853, 30533064 (2015).

6. Phillips, R., Kondev, J., Theriot, J. \& Garcia, H. G. Physical Biology of the Cell Second edition (Garland Science Taylor \& Francis Group, London and New York, 2013).

7. Galou, M. et al. The Importance of Intermediate Filaments in the Adaptation of Tissues to Mechanical Stress. Evidence from gene knockout studies. 89, 85-97 (1997).

8. Block, J. et al. Nonlinear Loading-Rate-Dependent Force Response of Individual Vimentin Intermediate Filaments to Applied Strain. Phys. Rev. Lett. 118, 048101 (2017).

9. Block, J. et al. Viscoelastic properties of vimentin originate from nonequilibrium conformational changes. Sci. Adv. 4, eaat1161 (2018). 


\section{Chapter 2}

\section{State of the Art}

\subsection{Intermediate Filaments}

Comparing MFs, MTs, and IFs on the genetic and structural level already indicates that IFs have a special role within the cell. While MFs and MTs are highly conserved along cell types and species, at least 70 genes in human are known to encode different types of IFs [1] and a lot more IF genes were found in other vertebrate and invertebrate [2]. All of these IFs are expressed in a cell type specific manner. Typically, epithelial cells express keratins, while mesenchymal cells are characterized by a network of vimentin IFs and neurons have IFs made of three neurofilament proteins [3]. The nucleus of higher metazoan possesses an additional IF system made of lamins which form the inner linning of the nucleus [3]. Via nuclear membrane proteins the lamin network is directly coupled to the particular cytoplasmic IF network [3].

By sequence homology, IF proteins are grouped into five classes (type I to type $\mathrm{V}$ or SHC 1 (sequence homology class) to SHC5) [4, 5], which also correspond to their biological origin and function. Table 2.1 shows the five SHCs and some examples for IFs in human according to Ref. [5]. 
Table 2.1: Sequence homology classes of intermediate filament proteins and their occurrence in mammals. Table according to and adopted from Ref. [5]

\begin{tabular}{|c|c|c|}
\hline Class & IF proteins & occurrence (in mammals) \\
\hline$\overline{\mathrm{SHC}} 1$ & / acidic cytokeratins & epithelial cells \\
\hline SHC 2 & basic cytokeratins & epithelial cells \\
\hline SHC 3 & $\begin{array}{l}\text { e.g. vimentin, desmin, glial fibril- } \\
\text { lary acidic protein (GFAP) }\end{array}$ & $\begin{array}{l}\text { mesenchymal cells, muscle cells, } \\
\text { astrocytes, glia cells }\end{array}$ \\
\hline SHC 4 & $\begin{array}{l}\alpha \text {-internexin, neurofilaments (NF- } \\
\mathrm{L}, \mathrm{NF}-\mathrm{M}, \mathrm{NF}-\mathrm{H} \text { ) }\end{array}$ & neurons \\
\hline SHC 5 & lamins (type A/C and B) & nucleus \\
\hline
\end{tabular}

\subsubsection{Architecture of Intermediate Filaments}

All IF proteins share a common secondary structure (see Figure 2.1, top left). The central $\alpha$-helical rod domain is conserved in size and in the specific pattern of its segments [6]. One exception concerning the size is the rod domain of the nuclear lamins, which shares the structure but has a slightly extended coil 1B, which contains 42 amino acids more than the other (cytoplasmic) IF types [2].

The rod domain is substructured into three coils ( $1 \mathrm{~A}, 1 \mathrm{~B}$, and 2$)$ connected by linkers (L1 and L12) [6]. The amino acid sequence follows, in general, a characteristic heptad repeat (abcdefg) $\mathrm{n}$, where position a and $\mathrm{d}$ are hydrophobic amino acids (e.g. Leu, Ile, Val, Met), which is typical for proteins that form a left-handed coiled-coil $[2,7,8]$. However, in some places this periodicity is different. In the beginning of coil 2 three hendecad repeats are found. Additionally one hendecad repeat is found in both, coil $1 \mathrm{~B}$ and the second half of coil $2[2,8,9]$. The hendecad repeat in the second half of coil 2 is traditionally called "stutter", as it causes a discontinuity within the coiled-coil structure. The hendecad repeat in the beginning of coil 2 led to a definition as coil $2 \mathrm{~A}$ and linker 2 in earlier structure predictions as the hendecad repeat was only developed as a motif for coiled coils in the late 90th of the last century [10].

X-ray crystallography of a fragment of a vimentin dimer (residues 328-411) revealed that the coiled-coil locally unwinds at the stutter region and the two $\alpha$-helices run parallel for a short distance [11]. The same behavior of the stutter region was found for lamin A [12] and keratin K5/K14 [13], indicating that this distinct structure is crucial for IFs [2]. Crytallography studies of vimentin coil 2 revealed a parallel run of the two 
$\alpha$-helices also for the hendecad repeat in the beginning of coil 2 [14]. In general, the hendecad repeat, compared to the ideal heptad repeat, leads to a non-optimal packing of the hydrophobic core of the coiled-coil and therefore to a thermodynamically less stable structure [9].

The rod is flanked by intrinsically disordered "head-" and "tail-" domains, which both differ considerable in size and amino acid sequence between the different IF proteins and between the same IF proteins of different species [2].

\subsubsection{Assembly of Intermediate Filaments}

The assembly of IFs differs profoundly from the formation of the other two cytoskeletal filament systems. MFs and MTs are built from globular subunits that polymerize in a polar way [16], while IF formation follows a strict hierarchical assembly mechanism. A schematic view of this process by the example of vimentin is shown in Fig. 2.1. Starting from the monomers, parallel coiled-coils, either as heterodimers (e.g. keratins) or as homodimers (e.g. vimentin), are formed. Two dimers come together to form antiparallel half-staggered, and due to their anti-parallel symmetry, non-polar tetramers [17]. At least up to this point, in vitro assembly occurs spontaneously due to reduction of denaturing agencies in physiological buffer conditions.

Unusually for proteins, keratins already form stable dimers even in $5 \mathrm{M}$ urea [18] and vimentin forms dimers in $6 \mathrm{M}$ urea and ordered tetramers in $5 \mathrm{M}$ urea [19], underlining the very strong attractive forces of these proteins. Hatzfeld and Franke also showed, that keratins from different cell types, that would never be co-expressed naturally, and even keratins from different species, do form dimers [18].

The formation of IFs, in contrast to MFs and MTs, does not require any additional energy, e.g. in terms of nucleoside-triphosphates. However, for in vitro assembly further than the tetramer state, the addition of ions or a change in $\mathrm{pH}$ is needed. Filament formation continues via lateral assembly of subunits into about $65 \mathrm{~nm}$ long [19] unitlength filaments (ULF), containing an average number of tetramers that depends on the IF type. Even though, the assembly occurs in this highly ordered manner, the number of tetramers per cross-section differs not only between different IF types. Mass-perlength measurements revealed that the number of subunits per cross-section varies between filaments of the same IF type [2, 19-22] and even along one filament [21, 22]. 


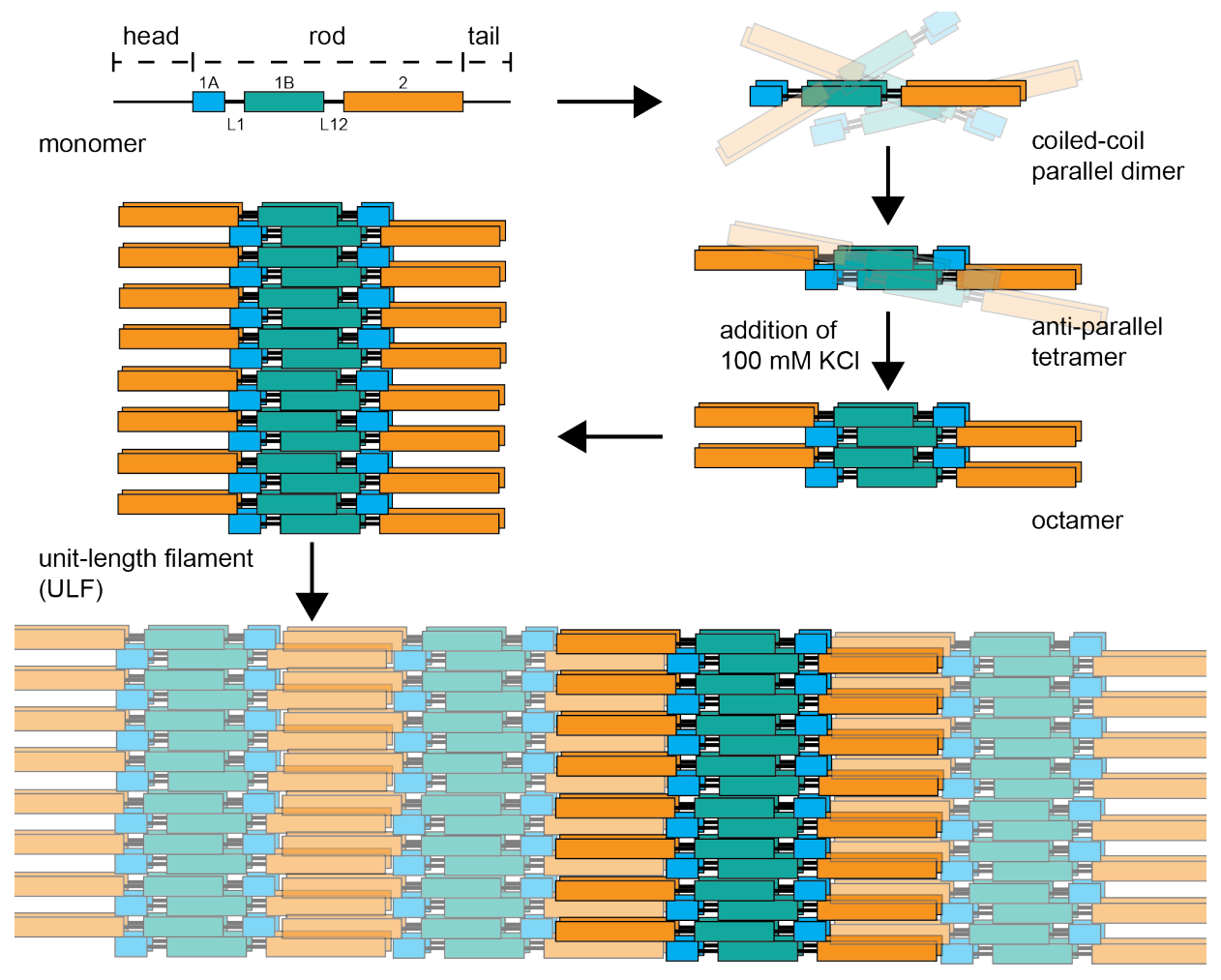

Figure 2.1: Schematic view of the assembly of vimentin intermediate filaments, from the monomer to mature filament. For simplicity structures are drawn as sheets. The assembly starts from the monomer. Two monomers form a parallel coiled-coil dimer and two dimers form an anti-parallel half staggered tetramer. The parallel assembly of ULFs yields unit length filaments which finally assemble longitudinal and form elongated filaments. Some IFs possess a final compaction step. Parts of this figure are reproduced from reference [15] with the permission of the American Physical Society under the terms of the Creative Commons Attribution 3.0 License.

It was shown for vimentin that this "polymorphism" can be influenced by the method used for initiation of assembly. When salt is added instantaneously, filaments are more polymorphic than filaments that are assembled by dialysis into a salty buffer [19].

Finally ULFs come together and form $\mu \mathrm{m}$-long filaments by longitudinal annealing. Due to the non-polarity and the special architecture of IFs, additional assembly mechanisms are possible. End-to-end annealing of two assembled filaments was observed 
in vivo $[23,24]$ and in vitro $[25,26]$. Another observed phenomenon is the exchange of subunits from mature filaments. Again this was found in vitro [26] and in cell experiments [23, 27]. However, both processes were shown to be very slow [25, 26]. Some IFs, e.g. vimentin and desmin undergo a final compaction step to form the mature filament [17].

The assembly speed is influenced by protein concentration and present ions, as well as temperature [20]. The general assembly mechanism was revealed by electron microscopy [19, 20] and atomic force microscopy [28, 29] and showed a ULF formation of vimentin within seconds and very short filaments within minutes [28]. More recently, small angle X-ray scattering was used to gain deeper knowledge about the structure of IFs [30,31] and in combination with microfluidic systems confirmed a ULF formation of vimentin on a time scale of a second [32]. For vimentin it was shown that the assembly speed is about $0.2 \mathrm{~nm} \cdot(\mu \mathrm{M} \cdot \mathrm{s})^{-1}$ and therefore proportional to the protein concentration $[17,29]$. Even though this has not been investigated as systematic for keratins, individual values suggest an assembly speed of about $23 \mathrm{~nm} \cdot(\mu \mathrm{M} \cdot \mathrm{s})^{-1}$ [33], while it is in the range of a few $\mathrm{nm} \cdot(\mu \mathrm{M} \cdot \mathrm{s})^{-1}$ for MFs and MTs $[17,34,35]$.

\subsubsection{Properties of Intermediate Filaments - Similar, but not Identi- cal}

Even though, all IFs share the secondary structure, they do not share all properties. Some differences were already pointed out above, e.g. the different preferences in $\mathrm{pH}$ and ionic strength for assembly. The tendency to form homo- or heterodimers and the variations in the number of subunits per ULF. Further differences were found in the persistence length of different IFs, in charge- and hydrophobicity patterns and in the formation of networks both in vitro and in vivo [17]. A detailed description of how properties differ between different IFs can be found e.g. in references $[2,3,6,17,36$, $37]$. 


\subsection{Intermediate Filaments under Load}

The outstanding features of hard- $\alpha$-keratin in form of e.g. wool or human hair aroused the interest of scientist already in the beginning of the 20th century. And even though the experimental setups were not as sensitive and precise as today, as early as in 1924 Shorter explained the experimentally found elastic behavior of wool using a two-spring model - with one spring acting free and one spring acting in a viscous medium [38]. This model already covered different mechanical regimes depending on the applied strain and also showed hysteresis. Experimentally, he showed that wool, which was stretched to a strain of not more than 0.4 , fully recovers in length upon relaxation and that this process is faster when the wool is wet [38]. He also showed that once extended fibers do not immediately recover structurally. Directly repeated load-extension curves of the same fiber yielded a higher extensibility with lower tension. Only after staying unstrained for several days the first load-extension curve was reproducible [38].

In 1927 John B. Speakman found the extensibility and the breaking load of wool fibers to be loading rate dependent. From microscopic observations he concluded that the hierarchical structure of wool is the reason for its elasticity and extensibility. Assuming the wool fiber to behave like a single elastic cell, filled with a fibrillar structure surrounded by a viscous medium, he hypothesized the elastic small strain extensibility to be due to stretching the elastic cell wall which is acting as a Hookean spring. By exceeding a critical strain the fibrillar structure comes into play which extends by the rotation of the fibrillae until they are all drawn into line. The softening of the wool fiber is then due to breaking of fibrillae, while the recovery of fiber length upon relaxation is due to the remaining intact fibrillae and the cell wall, which are pulling the fiber back to its initial length [39]. However, at this point this was speculation and a satisfactory proof was missing.

Some years later, William T. Astbury, who was a pioneer exploring the structure of biological macromolecules using x-ray measurements, found an essential transformation in the x-ray pattern of unstretched and stretched keratin by measuring the "Structure of Hair, Wool and Related Fibers" [40-42]. He referred to the x-ray photograph of stretched hair, which is comparable with that of natural silk, as $\beta$-keratin and that of unstretched hair as $\alpha$-keratin. From these findings he concluded that the difference 
between stretched and unstretched keratin is the structure of the polypeptide chain which is extended in the stretched and folded in the unstretched keratin [41]. A precise explanation on the molecular level was not possible before the definition of the $\alpha$-helix and the $\beta$-sheet by Pauling and Corey in the 1950ths $[43,44]$.

In 1960 Bendit continued this work studying the $\alpha$ - $\beta$-transition in wool keratin by $\mathrm{x}$-ray diffraction measurements. His experiments showed that the transition from the $\alpha$-state into the $\beta$-state starts at $5 \%$ extension or less and that the extension of the fiber and the extend of transformation are usually proportional [45].

More recently, the $\alpha$-helix to $\beta$-sheet transition was directly observed in vimentin as well. Using fibers made from hydrogels of in vitro assembled vimentin Pinto et al. performed wide angle x-ray scattering and found, subsequent to a single stretching event, peaks in the $\mathrm{x}$-ray pattern arising, which are typical for the spacing within $\beta$ sheets. They additionally found these peaks to be more intense when a cross-linker like magnesium chloride was present [46].

\subsubsection{Mechanics of Individual Intermediate Filaments}

IFs do form superstructures and networks due to several influencing factors, e.g. ions or cross-linking proteins [6, 17], and the mechanical properties of these structures were analyzed in a plethora of experiments, as partly reviewed above for bundles and superstructures. Articles reviewing the mechanical properties of IF networks can be found for example in references $[6,17,37]$. A comparison of studies on networks of IFs and on MFs and MTs, shows that IFs are much more stretchable than the other two cytoskeletal protein systems. This enormous elongation potential (up to a maximum of 3.6 fold [47]) is most likely encoded in the secondary structure of the monomers and in the hierarchical building up of the filaments as described above. Additional to the $\alpha$ helix to $\beta$-sheet transition, the slippage of subunits against each other [48-50] and the unfolding of the head- and tail-domains were suggested to contribute to the extension $[47,51]$. The $\alpha$ - $\beta$-transition is the only mechanism that was measured so far and is also consistent with the observed thinning of the filaments subsequently to the stretching $[47,52]$. However it can only account for a length increase of about $100 \%[47,53]$.

Probably due to experimental challenges, there are only very few publications about the mechanical properties of individual IFs. The usual experimental setup that was cho- 
sen for single IF measurements was atomic fore microscopy (AFM). IFs were assembled in vitro and put on a solid support where they adsorb to the surface. By driving an AFM tip laterally through the adsorbed IFs, the filaments were locally displaced and subsequently imaged to measure the elongation [47, 52], as shown in Fig. 2.2 A. Guzman et al. reduced the influence of the surface by using a solid support with $250 \mathrm{~nm}$ holes over which the filaments were spanned. With the AFM tip they deformed the filament into the hole and therefore tested the bending stiffness of vimentin IFs without a direct solid support at the bending position [50]. Due to the experimental setup, the mea-

A

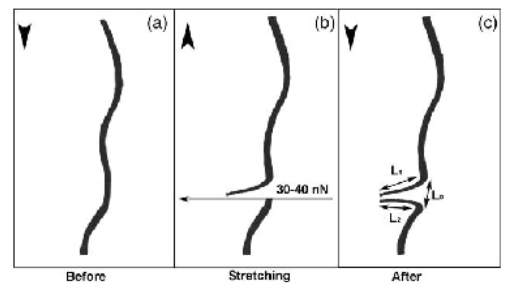

B

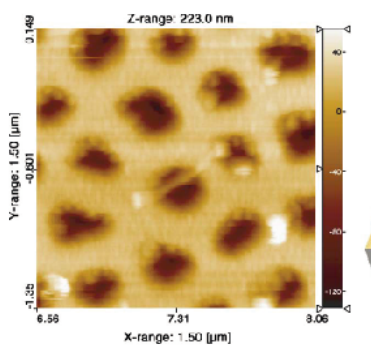

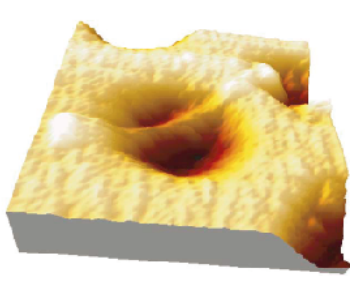

Figure 2.2: (A) Schematic representation of AFM experiment performed in reference [47]. IFs, adsorbed to a surface, are manipulated by an AFM tip that is moved through the filament along the arrow in (b). The filament is imaged by AFM tapping mode before (a) and after (c) the manipulation. Filament extension is calculated as depicted in (c). The figure is reprinted from reference [47] with permission of Elsevier. (B) (left) AFM image of a single vimentin filament spanned over a hole in the underlying substrate. (right) The same filament shown as $3 D$ representation. The figure is reprinted from reference [50] with permission of Elsevier.

surements in all three publications $[47,50,52]$ were performed on filaments not longer than $250 \mathrm{~nm}$, which is below the persistence length that was measured for different IF types [17]. As in the higher order structures, different stretching regimes were found also on the single filament level. IFs seem to be fully elastic at small strains $(\epsilon=0.1$ to 1$)[50,52]$ and forces (60 to $130 \mathrm{pN}$ ) [50], while a further extension was found to be plastic/irreversible and accompanied by strain hardening in AFM experiments [47]. However, the experiments were performed on a solid support which might also block, at least partly, an elastic or reversible behavior at higher strains [47].

Kiss et al. analyzed single desmin fibers adsorbed to a solid support by pressing an AFM tip on the surface at a position where a desmin filament was lying and subse- 
quently pulling it away from the surface [49]. This experimental procedure would allow for a stretching event without a solid support. Analyzing the imaged filaments subsequently to the stretching, they however concluded that they never removed an entire filament from the surface, but only desmin subunits from the filament [49].

A more recent study is the publication of Ramm et al. on a vimentin coiled-coil consisting only of the second part of vimentin coil 2 (former coil 2B). Using single molecule force spectroscopy they tested the stability and the folding of this part of the vimentin dimer by opening up the coiled-coil from both ends respectively [54]. Even though, this is not a study on a full filament, it provides interesting insights on the stability of the vimentin coiled-coil. Taking their results together, they divide the second part of vimentin coil 2 into three stability sections. While the coiled-coil is easily unzippable from the labile $\mathrm{N}$-terminal end, the middle part of the coiled-coil is medium stable and the C-terminal section highly stable. Ramm et al. hypothesize this stability profile to be important for assembly, as it may ensure that the coiled-coil stays zipped and is stable during the formation of tetramers and ULFs [54].

\subsubsection{Simulations of Intermediate Filament Mechanics}

To date, only very few articles were published dealing with simulations of IF mechanics. Such simulations are computationally very expensive and it is therefore not possible to exactly repeat stretching experiments, like the ones performed in the scope of this thesis, in a simulation. Notably the stretching velocity that is used in experiments 0.5 to $5 \mu \mathrm{m} / \mathrm{s}$ ) is, so far, not accessible in atomistic molecular dynamics simulations [55]. Nevertheless, the simulations that were published so far, provide highly interesting and helpful insights into the relationship between the structure of IF proteins and their mechanical properties.

The above explained study of the vimentin coiled-coil by Ramm et al. [54] was accompanied by in silico measurements. Mimicking their experiment in a simulation they aimed good agreement between in silico and in vitro results and used this to verify their experimental results [54].

Ackbarow et al. analyzed the mechanical properties of the vimentin coiled-coil part including the stutter region and predicted the stutter to be a predefined unfolding region. Comparing two parallel $\alpha$-helices with a coiled-coil and a coiled-coil including a 
stutter they found the stutter region to be the least resilient part of the structure [56]. By studying the coiled-coil further, they also found a pulling rate dependent deforming mechanism and hysteresis when stretching the vimentin coiled-coil cyclically [57]. From these results, they suggested two features to be encoded in the coiled-coil. For small pulling velocities and little deformations, the coiled-coil acts fully elastic, while at higher deformations and pulling rates it is able to dissipate energy [57].

Vimentin dimers and tetramers, as well as their mechanical behavior due to tensile load were simulated by Qin et al. [53, 58, 59]. By comparing their results to lab experiments performed with IFs [50,52], they found a good agreement of the results of the in silico and in vitro studies [53]. On the molecular level they found the predicted $\alpha$ $\beta$ transition and for the tetramer subsequent inter-dimer sliding. They also found the unfolding force of the $\alpha$-helix to be strongly pulling velocity dependent and suggested that the hierarchical structure of vimentin is crucial for its mechanical properties [53, 58].

Chou et al. performed atomistic simulations of human trichocyte keratin dimers and tetramers [60] as well as a mesoscopic coarse grained model of a keratin macrofilament fibril [61]. In both studies they found the disulfide bonds to be important for the strength and resilience. Antunes et al. also performed simulations of eight keratin chains of hair keratin and found the less coiled structures to deform first [62]. However, their data also showed considerable differences to stress-strain curves of in vitro experiments.

\subsubsection{Intermediate Filaments and the Mechanical Properties of Cells}

Apart from e.g. material sciences, one goal of illuminating the mechanical properties of IFs is to gain insights about their function within the cytoskeleton and their contribution to the mechanical properties of cells. The experimental design to answer this question is far more complex than the design of in vitro experiments as there are so many unknown elements within a cell. The most frequently used system is the comparison of a wild type cell line with a cell line that does not possess IFs anymore (knock out cell line). Another requirement is a suitable experimental setup to test the deformability, stiffness or rigidity of whole cells in a comparable way.

Using fluorescence microscopy, Fudge et al. found keratinocytes to survive strains 

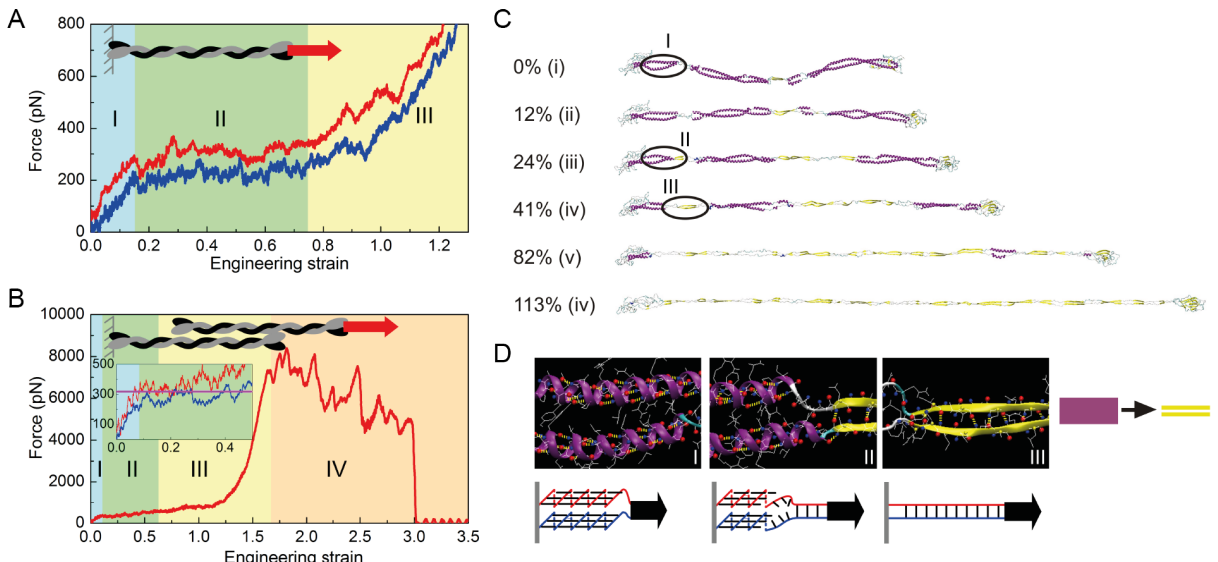

Figure 2.3: Results of simulated stretching events of vimentin dimers and tetramers. (A) Force-strain curves of a vimentin dimer at pulling velocities of $1 \mathrm{~m} / \mathrm{s}$ (blue) and $10 \mathrm{~m} / \mathrm{s}$ (red). Numbered and colored regimes indicate changes in molecular response: (I) linear stretching of a spring, (II) $\alpha$-helix unfolding, (III) stiffening and pulling the protein backbone of the dimer. (B) Force-strain curve of a vimentin tetramer at a pulling velocity of $10 \mathrm{~m} / \mathrm{s}$. The inlay shows the force-strain curves at pulling velocities of $1 \mathrm{~m} / \mathrm{s}$ (b/ue) and $10 \mathrm{~m} / \mathrm{s}$ (red) for a strain smaller than 0.5. The pink line indicates the unfolding force of the dimer. The numbered and colored regimes are interpreted as: (I) stretching of a spring, (II) unfolding of segment 2B, (III) unfolding of the whole protein and (IV) interdimer sliding. (C) Possible molecular mechanism of the proposed $\alpha$-helix to $\beta$-sheet transition as a function of applied tensile strain. (D) Snapshots of the atomistic details of the $\alpha$-helix to $\beta$-sheet transition of the right part of coil 1 A of a vimentin dimer (as indicated in (C)) Potential rupture and new formation of $\mathrm{H}$-bonds are sketched below the snapshots. This figure is reprinted from reference [53] under the Creative Commons Attribution (CC BY) license.

as high as $133 \%$ and that a strain of $100 \%$ only caused minor damages in the keratin network [63]. Ramms et al. analyzed the mechanical properties of keratinocytes by AFM and magnetic tweezers and found keratin lacking cells to be softer and showing a higher viscous contribution to the displacement of magnetic beads [64]. They also showed that actin and MTs were not affected by the absence of keratin and that the reexpression of keratin $\mathrm{K} 5 / \mathrm{K} 14$ did almost restore the properties that were measured for the control cell line [64]. Optical stretcher experiments, invasion assays and 3D growth assays, revealed murine keratinocytes with a keratin knock out to be $60 \%$ more deformable compared to cells were the actin network was disrupted by a Latrunculin A treatment. The increased invasiveness due to a keratin knock out was recovered after the reexpression of a small amount of keratin $\mathrm{K} 5 / \mathrm{K} 14$, while the cells still showed in- 
creased deformability [65]. With the same cell line Seltmann et al. also showed keratin knock out cells to adhere faster to the extracellular matrix and migrate two times faster and with reduced directionality compared to the wild type cells and the rescue cell line, which reexpressed keratin K5/K14 [66].

For T-lymphocytes it is important to be rigid while circulating with the blood flow, but to be highly flexible when entering the tissue [67]. Brown et al. hypothesize that vimentin filaments that form a cage like structure in the circulating lymphocyte and collapse into an aggregate like structure to exit the vasculature are important for this cell type to control its deformability [67].

Investigating vimentin IFs in living cells, Wang et al. tested the stiffness due to applied strain in vimentin deficient mouse embryonic fibroblasts by magnetic twisting cytometry. They found the vimentin deficient cells to be less stiff compared to the wild type cells. The vimentin deficient cells also showed decreased stiffening due to applied strain, except for the lowest stress they applied [68]. They additionally observed a slower proliferation and DNA synthesis in the vimentin deficient cells [68]. Guo et al. measured little contribution of the vimentin IFs to the cortical stiffness in embryonic mouse fibroblasts but found a role for vimentin in regulating intracellular mechanics. In their active microrheology experiments they revealed increased mechanical integrity due to vimentin and showed that vimentin contributes to the localization of intracellular components [69]. Mendez et al. analyzed how vimentin influences the cell shape due to the expression or knock out of the vimentin network. After the formation of a vimentin network, epithelial cells adopted a mesenchymal cell shape and showed increased adhesion and mobility. The disruption of the vimentin network in mesenchymal cells in contrast led to an epithelial cell shape [70]. Following up on this work they analyzed the behavior of wild type and vimentin-null mouse embryonic fibroblasts on substrates of different stiffness and in a 3D matrix. Concluding their results, they hypopthesize a role for vimentin in the protection against compressive stress, mechanical integrity and the elasticity of cells [71].

Even though this review of IF research makes no claim to completeness, to the best of my knowledge a study of the mechanics of individual, freely fluctuating IFs, under physiological buffer conditions, was not yet published (except the publications within the scope of the present thesis). 


\subsection{Mathematical Models for Biopolymers}

Polymers are important building blocks of biological matter. Not only the filaments that form the cytoskeleton, but also the DNA, which is a sequence of nucleotides, and proteins in general, which are sequences of amino acids, are important polymer types. In order to describe and compare the properties of such polymers, different physical concepts are used. Key criteria in choosing the right theory are the basic characteristics of a polymer, such its flexibility and the structure of its building blocks, the properties to be described (micro- or macroscopic), as well as the needed degree of accuracy. Two basic and widely used concepts to describe biopolymers are the freely-jointed chain (FJC) and the worm-like chain (WLC) model (see Fig. 2.4).

A

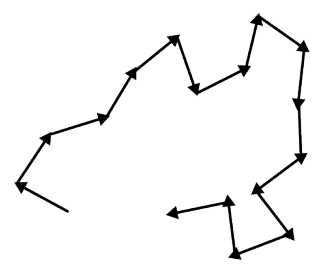

B

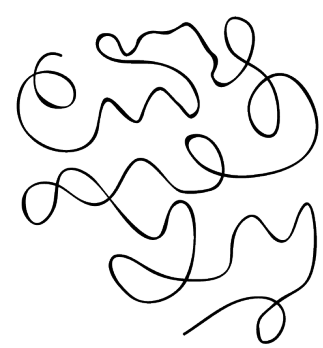

Figure 2.4: A: A freely-jointed or ideal chain, B: A worm-like chain. Adopted from [72]

\subsubsection{Freely-jointed Chain and Worm-like Chain}

The FJC, also referred to as ideal chain, random walk chain or Gaussian chain, is the simplest model to describe a polymer [72]. In this model, any kind of interaction between the monomers of the polymer are neglected and each monomer is treated as a point connected to the next monomer by a linker of step length $l$. The total unfolded length or contour length $L_{\mathrm{c}}$ of such a polymer of $N$ links would then simply be [72]:

$$
L_{\mathrm{C}}=N \cdot l=N \cdot\left|\boldsymbol{l}_{\mathrm{i}}\right|
$$


with $\boldsymbol{l}_{\mathrm{i}}$ being the link vector between two subsequent monomers. Summing up all $\boldsymbol{l}_{\mathrm{i}}$ results in the end-point vector $\boldsymbol{l}_{\mathrm{E}}$ :

$$
\boldsymbol{l}_{\mathrm{E}}=\sum_{\mathrm{i}=1}^{\mathrm{N}} \boldsymbol{l}_{\mathrm{i}}
$$

Since the scalar product with itself yields the square of a length of a vector, the mean square end-to-end distance $\left\langle R^{2}\right\rangle$ can be calculated from:

$$
\left\langle R^{2}\right\rangle=\left\langle\boldsymbol{l}_{\mathrm{E}} \bullet \boldsymbol{l}_{\mathrm{E}}\right\rangle
$$

with $\langle$ indicating the ensemble average [72]. Based on the geometrical definition of the scalar product $\boldsymbol{a} \bullet \boldsymbol{b}=|\boldsymbol{a}| \cdot|\boldsymbol{b}| \cdot \cos \theta_{\mathrm{ab}}$ (see Fig. 2.5 B), equation 2.3 can be written as:

$$
\left\langle R^{2}\right\rangle=\left\langle\sum_{\mathrm{i}=1}^{\mathrm{N}} \boldsymbol{l}_{\mathrm{i}} \cdot \sum_{\mathrm{j}=1}^{\mathrm{N}} \boldsymbol{l}_{\mathrm{j}}\right\rangle=N \cdot l^{2}+2 \cdot l^{2} \cdot \sum_{\mathrm{i}=1}^{\mathrm{N}-1} \sum_{\substack{j=2 \\ \mathrm{i}<\mathrm{j}}}^{N}\left\langle\cos \theta_{\mathrm{ij}}\right\rangle
$$

with $\left\langle\boldsymbol{l}_{\mathrm{i}} \bullet \boldsymbol{l}_{\mathrm{j}}\right\rangle=\boldsymbol{l}^{2} \cdot\left\langle\cos \theta_{\mathrm{ij}}\right\rangle$ and $\theta$ being the angle between to subsequent steps $l_{\mathrm{i}}$ and $l_{\mathrm{j}}[72]$. As defined above, steps in the FJC are uncorrelated $\left(\left\langle\boldsymbol{l}_{\mathrm{i}} \bullet \boldsymbol{l}_{\mathrm{j}}\right\rangle=0\right.$ and $\left.\left\langle\cos \theta_{\mathrm{ij}}\right\rangle=0\right)$, therefore equation 2.4 is simplified to [72]:

$$
\left\langle R^{2}\right\rangle=N \cdot l^{2}
$$

For the case where orientation correlation is not neglected, the mean square end-toend distance can be calculated from equation 2.4. For a correlation over a distance of $m$ monomers, the second term of equation 2.4 is approximately [72]:

$$
\sum_{\mathrm{i}=1}^{\mathrm{N}-1} \sum_{\substack{j=2 \\ \mathrm{i}<\mathrm{j}}}^{N}\left\langle\cos \theta_{\mathrm{ij}}\right\rangle \simeq \sum_{\mathrm{i}=1}^{\mathrm{N}-1} \sum_{\substack{j=2 \\ \mathrm{i}<\mathrm{j}<\mathrm{i}+\mathrm{m}}}^{N}\left\langle\cos \theta_{\mathrm{ij}}\right\rangle
$$

In a homogeneous chain without end effects this assumption is true independent on $i$ 
A

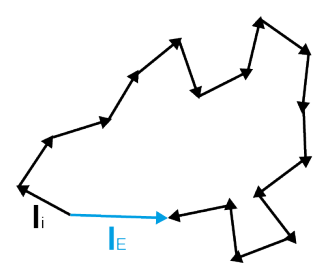

B

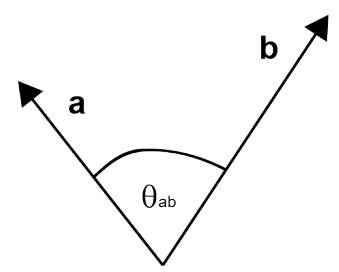

C

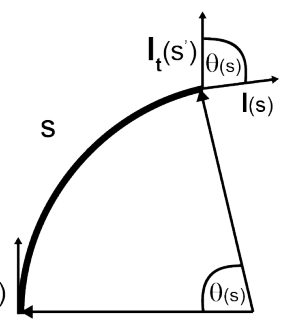

Figure 2.5: (A) Schematic representation of a FJC with the link vector $\boldsymbol{l}_{i}$ and the end-point vector $\boldsymbol{l}_{E}$. Adapted from [72]. (B) Geometrical scalar product $\boldsymbol{a} \cdot \boldsymbol{b}=|\boldsymbol{a}| \cdot|\boldsymbol{b}| \cdot \cos \theta_{a b}$. (C) Representation of a part of an elastic filament with a constant curvature $\theta / s$ and the tangent vectors $\boldsymbol{l}_{t}(s)$. Adapted from [72]

and equation 2.6 can be rewritten as [72]:

$$
\sum_{\mathrm{i}=1}^{\mathrm{N}-1} \sum_{\substack{j=2 \\ \mathrm{i}<\mathrm{j}<\mathrm{i}+\mathrm{m}}}^{N}\left\langle\cos \theta_{\mathrm{ij}}\right\rangle \simeq N \cdot \sum_{j=2}^{N}\left\langle\cos \theta_{\mathrm{ij}}\right\rangle
$$

The mean square end-to-end distance for a chain with short range orientation can therefore be calculated from:

$$
\left\langle R^{2}\right\rangle=N \cdot l^{2} \cdot \sigma
$$

with $\sigma=1+2 \cdot \sum_{j=2}^{m} \cos \left(\theta_{1 j}\right)$ [72]. Renormalizing the number of segments $N$ and the step length $l$ by the factor $\sigma$ yields the Kuhn chain with step length $l_{\mathrm{K}}=\sigma \cdot l$ and number of segments $N_{\mathrm{K}}=\frac{N}{\sigma}$ [72]. This renormalization eliminates the effects of the introduced orientation correlation by rewriting the equations for the contour length and the mean square end-to-end distance [72]:

$$
\begin{gathered}
L_{\mathrm{c}}=N \cdot l=N_{K} \cdot l_{\mathrm{K}} \\
\left\langle R^{2}\right\rangle=N_{\mathrm{K}} \cdot l_{\mathrm{K}}^{2}
\end{gathered}
$$

From comparison of equation 2.1 and equation 2.9 it can be seen that the Kuhn-chain is equivalent to the FJC, but with a step length $l_{\mathrm{K}}$ that is a measure for the filaments 
stiffness and does not directly coincide with the size of the monomers.

To transfer the FJC into a WLC, the step length tends to zero while the number of monomers tends to infinity, yielding a filament that is continuously flexible.

A typical number to describe the stiffness or the flexibility of a polymer is the socalled persistence length, which is defined as the length over which the filaments orientation correlation is lost [72]. In a FJC the persistence length $L_{\mathrm{P}}$ equals the step length $l$, while for a rod-like chain the persistence length is equal to or even longer than the contour length. Fig. 2.5C shows a segment of an elastic filament of length $s$, which elastic bending energy can be described following Hook's law:

$$
\Delta U=\frac{1}{2} \cdot s \cdot \kappa \cdot\left(\frac{\theta}{s}\right)^{2}
$$

with $\theta$ being the angle between the two tangent vectors separated by the filament length $s, \frac{\theta}{s}$ being the curvature and the bending rigidity constant $\kappa$ [72]. Equation 2.11 can be used to calculate the mean square bending angle $\left\langle\theta^{2}\right\rangle$ as an ensemble average at thermal equilibrium [72]:

$$
\left\langle\theta^{2}\right\rangle=2 \cdot \frac{\int \exp \left[\frac{-\Delta U}{k_{\mathrm{B}} T}\right] \cdot \theta^{2} \mathrm{~d} \theta}{\int \exp \left[\frac{-\Delta U}{k_{\mathrm{B}} T}\right] \cdot \mathrm{d} \theta}=2 \cdot \frac{s}{\kappa} \cdot k_{\mathrm{B}} T
$$

As described above, the orientation correlation $\langle\cos \theta(s)\rangle$ of a WLC decreases when two tangent vectors are separated by a distance $s \gg L_{\mathrm{P}}$ along the filament's contour. This decrease in orientation correlation with increasing distance $s$ can be described by an exponential decay [72]:

$$
\langle\cos \theta(s)\rangle=\exp \left(-\frac{|s|}{L_{\mathrm{P}}}\right)
$$

For distances $s \ll L_{\mathrm{P}}$ and therefore small angles $\theta$, it is possible to approximate both, the cosine and the exponential, by series expansion up to the first order [72]:

$$
1-\frac{1}{2} \cdot\left\langle\theta^{2}(s)\right\rangle \approx\langle\cos \theta(s)\rangle=\exp \left(-\frac{|s|}{L_{\mathrm{P}}}\right) \approx 1-\frac{s}{L_{\mathrm{P}}}
$$

From this approximation it follows that $\left\langle\theta^{2}(s)\right\rangle=2 \cdot \frac{s}{L_{\mathrm{P}}}$ and therefore $L_{\mathrm{P}}=\frac{\kappa}{k_{\mathrm{B}} T}$ [72].

To calculate the end-to-end vector $\boldsymbol{R}$ and the mean square end-to-end distance $\left\langle R^{2}\right\rangle$ 
of a WLC the sum over segments - as performed for the FJC - is changed into an integral over the contour length [72]:

$$
\boldsymbol{R}=\int_{0}^{L_{\mathrm{c}}} \boldsymbol{l}_{t}(s) \mathrm{d} s
$$

with $\boldsymbol{l}_{t}(s)$ being the tangent unit vector, and:

$$
\begin{aligned}
\left\langle R^{2}\right\rangle & =\int_{0}^{L_{\mathrm{c}}} \boldsymbol{l}_{t}(s) \mathrm{d} s \cdot \int_{0}^{L_{\mathrm{c}}} \boldsymbol{l}_{t}\left(s^{\prime}\right) \mathrm{d} s^{\prime} \\
& =\int_{0}^{L_{\mathrm{c}}} \int_{0}^{L_{\mathrm{c}}}\left\langle\cos \theta\left(s-s^{\prime}\right)\right\rangle \mathrm{d} s \mathrm{~d} s^{\prime} \\
& =\int_{0}^{L_{\mathrm{c}}} \int_{0}^{L_{\mathrm{c}}} \exp \left[\frac{-\left|s-s^{\prime}\right|}{L_{\mathrm{P}}}\right] \mathrm{d} s \mathrm{~d} s^{\prime} \\
& =2 L_{\mathrm{P}}^{2} \cdot\left[\frac{L_{\mathrm{c}}}{L_{\mathrm{P}}}-1+\exp \left(-\frac{L_{\mathrm{c}}}{L_{\mathrm{P}}}\right)\right]
\end{aligned}
$$

with the two limiting situations $L_{\mathrm{c}} \ll L_{\mathrm{P}}$ and $L_{\mathrm{c}} \gg L_{\mathrm{P}}$. While the first one describes a rigid $\operatorname{rod}\left(\left\langle R^{2}\right\rangle=L_{\mathrm{c}}^{2}\right)$, the second one is the recovery of a FJC $\left(\left\langle R^{2}\right\rangle=2 \cdot L_{\mathrm{c}} \cdot L_{\mathrm{P}}\right)$. Comparing the recovery of the FJC to equation 2.10 one reveals the relation $l_{\mathrm{K}}=2 \cdot L_{\mathrm{P}}$. This also means that, according to the definition of the Kuhn segments, there is no orientation correlation after twice the persistence length of a filament [72].

\subsubsection{Entropic Springs}

Counterintuitively, polymers, though being loosely collapsed (cf. Fig. 2.4) do exert a mechanical force due to stretching [73]. This behavior is explained by a reduction of entropy due to a reduced number of possible configurations in the stretched state compared to the completely relaxed state. Such a behavior is called entropic elasticity or entropic spring [73]. For small displacements both, the FJC and the WLC, act as ideal springs with spring constant $3 k_{\mathrm{B}} T / l L_{\mathrm{c}}$ and $3 k_{\mathrm{B}} T / L_{\mathrm{P}} L_{\mathrm{c}}$, respectively [73]. Important to point out is the fact that the spring constants of both the FJC and the WLC are dependent on the temperature.

For a FJC the force extension correlation can be estimated from [15, 74]:

$$
x=L_{\mathrm{c}} \cdot\left(\operatorname{coth}\left(\frac{F \cdot l}{k_{\mathrm{B}} T}\right)-\frac{k_{\mathrm{B}} T}{F \cdot l}\right)
$$


For high forces this equation can be further approximated using $\operatorname{coth}\left(\frac{F \cdot l}{k_{\mathrm{B}} T}\right)=1$ :

$$
x=L_{\mathrm{c}} \cdot\left(1-\frac{k_{\mathrm{B}} T}{F \cdot l}\right)
$$

A widely used approximation for the correlation between extension $x$ and force $F$ for a WLC is $[73,75,76]$ :

$$
\frac{F \cdot L_{\mathrm{P}}}{k_{\mathrm{B}} T}=\frac{1}{4 \cdot\left(1-\frac{x}{L_{\mathrm{c}}}\right)^{2}}-\frac{1}{4}+\frac{x}{L_{\mathrm{c}}}
$$

At high forces and therefore large extensions $\left(x \rightarrow L_{\mathrm{c}}\right)$ the quadratic term dominates the force-extension behavior of the WLC model:

$$
\frac{F \cdot L_{\mathrm{P}}}{k_{\mathrm{B}} T} \approx \frac{1}{4 \cdot\left(1-\frac{x}{L_{\mathrm{c}}}\right)^{2}}
$$

Solving equation 2.20 for extension $x$ results in:

$$
x=L_{\mathrm{C}} \cdot\left(1-\frac{1}{2}\left(\frac{k_{\mathrm{B}} T}{F \cdot L_{\mathrm{P}}}\right)^{\frac{1}{2}}\right)
$$

\subsubsection{Two-State Model}

For biopolymers that undergo conformational changes due to applied strain, models are needed that take the change in contour length and the kinetics of the conformational transition into account. One model, which was first introduced by Burte and Halsey to explain the elongation of wool fibers [77], and in a refined version shown to reproduce the experimental data for various biopolymers [76], is an elastically coupled two-state model. For a polymer which consists of monomers or modules that undergo a transition from state $a$ (low energy state) to a state $b$ (high energy state) due to an applied strain, this model is summarized in the following paragraphs for the transition from the $a$ to the $b$ state [76]. The transition from the $b$ state to the $a$ state can be modeled in analogy to [76]. The contour length $L_{\mathrm{c}}$ of such a polymer is the sum of the monomers in the $a$-state $N_{a}$ times the length of the $a$-state $l_{a}$ and the monomers in 
the $b$-state $N_{b}$ times the length of the $b$-state $l_{b}[76]$ :

$$
L_{\mathrm{c}}=N_{a} \cdot l_{a}+N_{b} \cdot l_{b}
$$

The transition rate $\alpha$ from one state into another is given by [76]:

$$
\alpha=\omega \cdot \exp \left[-\frac{\Delta E}{k_{\mathrm{B}} T}\right]
$$

with $\omega$ being the natural oscillating frequency [78] and $\Delta E$ being the energy barrier between the two states as indicated in Fig. 2.6. Assuming a Bell-Evans kinetic [78, 79]

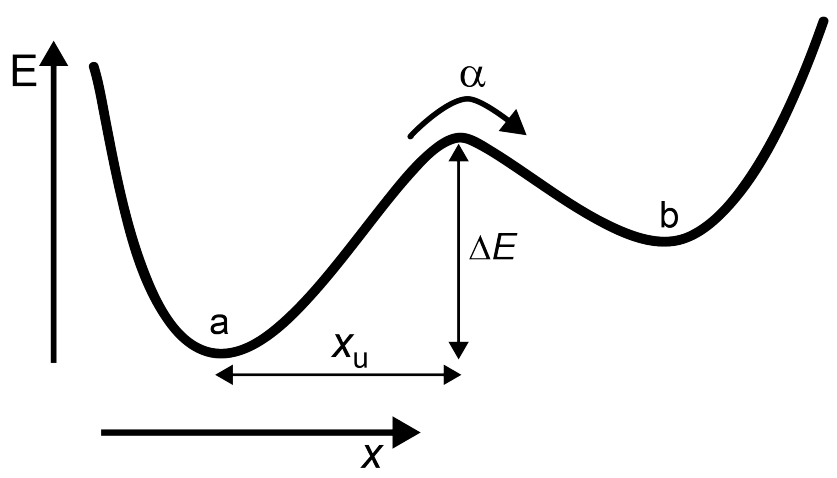

Figure 2.6: Sketch of a double-well potential which is assumed to describe the transition between two energetically different states of the same module of a polymer. $\Delta E$ is the energy barrier, $x_{u}$ the potential width of the energy barrier for the transition from $a$ to $b$.

the transition rate becomes force dependent [76]:

$$
\alpha(F)=\omega \cdot \exp \left[-\frac{\Delta E-F \cdot x_{\mathrm{u}}}{k_{\mathrm{B}} T}\right]=\alpha \cdot \exp \left[\frac{F \cdot x_{\mathrm{u}}}{k_{\mathrm{B}} T}\right]
$$

where the energy barrier is, according to Bell $[76,78]$, linearly reduced by the applied force $F$ times the width of the energy barrier $x_{\mathrm{u}}$.

The extension $x$ of the polymer is the product of stretching velocity $v$ and time $t$ [76]:

$$
x=v \cdot t
$$


From equation 2.19 the applied force $F$, corresponding to the extension $x$, can be calculated and used to determine the probability $\mathrm{d} P_{a}$ that modules of the polymer undergo the transition from state $a$ to state $b$ [76]:

$$
\mathrm{d} P_{a}=N_{a} \cdot \alpha(F) \cdot \mathrm{d} t
$$

Not all polymers are made from a single chain of monomers like e.g. DNA. In the case of IFs a single filament contains several monomers in parallel, as described in detail in Section 2.1.2. It was shown by Friddle et al. that the described two-state model is still suitable to describe the transition behavior remarkably accurate [80]. According to their calculations, the potential width $x_{\mathrm{u}}$ is an apparent value as it is reduced depending on the number of parallel bonds (or monomers that undergo a transition) [15, 80].

Detailed information on how these models were used and modified to fit and simulate the stretching of single vimentin IFs can be found in Sections 4.6 and 5.5.

\subsection{Optical Tweezers}

"Accelerating and trapping of particles by radiation pressure" [81] was the seminal publication by Arthur Ashkin, which led towards a new standard tool for biophysical research [82]. Optical tweezers or optical traps (OTs) are based on the principle that dielectric particles, e.g. glass or polystyrene beads, experience the so called gradient force when placed in the focus of a laser beam [83]. The first OT - stable in three dimensions - was reported by Ashkin et al. [84] in 1986. In the following years OTs were used to study, for example, single biological molecules, living cells, mechanical forces and elasticity of polymers and cells, folding properties of polymers such as DNA and the force generation as well as the kinetics of motor proteins [82]. Today, OTs are commercially available and the combination with other techniques, such as high resolution fluorescence microscopy, still opens up new possibilities for experiments. 


\subsubsection{Physics of Optical Trapping}

The physics behind the retaining of a particle or bead in the focus of a laser beam and its position restoring capability is most intuitively described by ray optics, momentum conservation and Snell's law [85]. The optical momentum from refraction of the laser beam in a trapped, transparent bead depends on its displacement from the laser focus. While the momenta of all incident rays sum up to zero, for a bead that is perfectly located in the focus of the laser beam, a slightly displaced bead will experience a restoring force $\Delta \boldsymbol{k}$ due to momentum conservation (see Fig. 2.7). The magnitude of

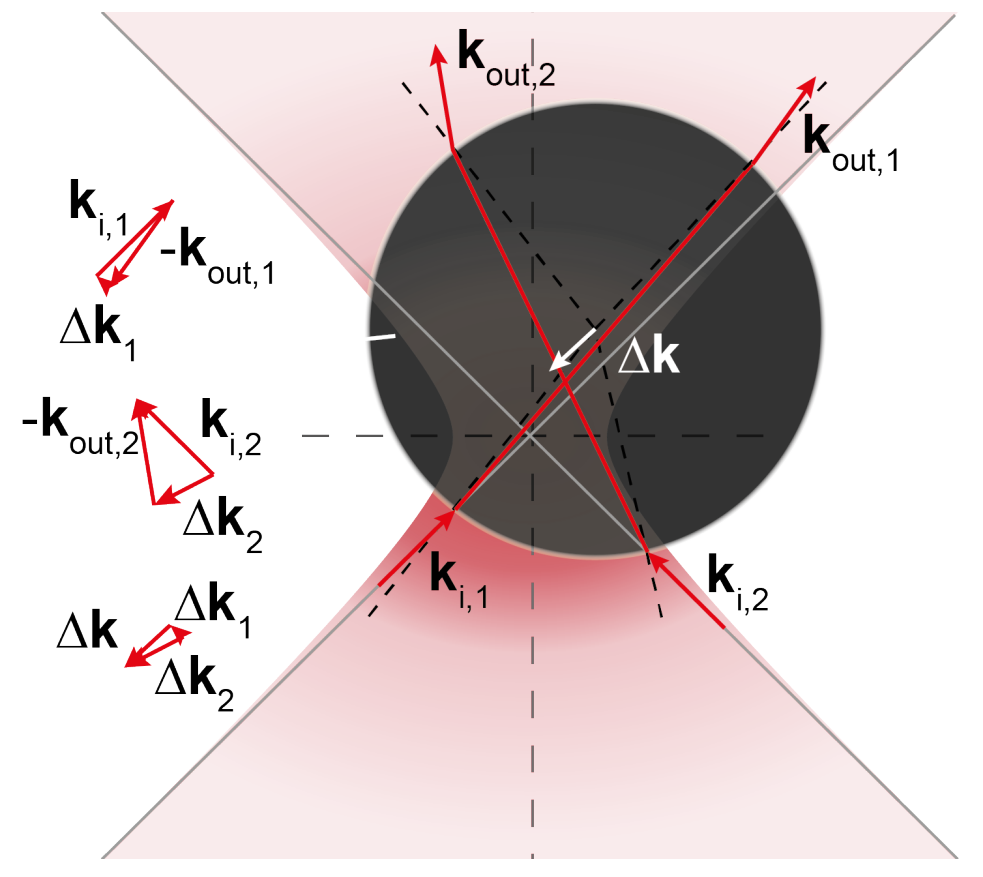

Figure 2.7: Ray Optics in a highly focused laser beam. Adapted from reference [85]

refraction of an incident ray $\boldsymbol{k}_{\mathrm{i}}$ depends on the difference in the refractive indices of the bead (typically glass or polystyrene) $n_{\text {bead }}$ and its environment (typically aqueous solution) $n_{\mathrm{env}}$ as well as its angle of incidence $\theta_{0}$. The refraction of an incident wave vector $\boldsymbol{k}_{\mathrm{i}}$ at the bead's surface results in a different outgoing wave vector $\boldsymbol{k}_{\text {out }}$ beyond 
the surface and these are connected by Snell's law:

$$
\boldsymbol{k}_{\mathrm{i}}=\boldsymbol{k}_{0} \cdot n_{\mathrm{env}} \cdot \sin \left(\theta_{\mathrm{i}}\right)=\boldsymbol{k}_{0} \cdot n_{\text {bead }} \cdot \sin \left(\theta_{\mathrm{o}}\right)=\boldsymbol{k}_{\text {out }}
$$

with $\left|\boldsymbol{k}_{0}\right|=\frac{2 \pi}{\lambda}$ being the wave vector in vacuum.

A ray of light obeys this rule every time it traverses a surface of two media with different refractive indices i. e. twice while transiting the bead. Unless the ray hits the bead's surface exactly perpendicular, the incident wave vector $\boldsymbol{k}_{\mathrm{i}}$ and the wave vector scattered from the other side of the bead $\boldsymbol{k}_{\mathrm{s}}$ are different and a resulting optical momentum $\Delta \boldsymbol{k}=\boldsymbol{k}_{\mathrm{i}}-\boldsymbol{k}_{\mathrm{s}}$ remains which acts as restoring force for displaced beads.

The ray optics picture holds true for large particles, with sizes much larger than the laser wavelength $\lambda$, which for OTs typically is about $1 \mu \mathrm{m}$. A more sophisticated approach to the physics of OTs is the so called Rayleigh approximation, whereby the particle is assumed to be much smaller than the wavelength of the laser, acting as a simple dipole, when placed in the focus of a laser beam [86]. The total optical force $F_{\mathrm{o}}$ acting on the particle has two components, the scattering force $F_{\mathrm{S}}$ and the gradient force $F_{\mathrm{g}}$ and can be calculated by integrating over the whole volume of the trapped particle (scatterer).

$$
F_{\mathrm{o}}=F_{\mathrm{s}}+F_{\mathrm{g}}=\int_{V} f \mathrm{~d} V
$$

There is no simple possibility to calculate $F_{\mathrm{s}}$, but it can be assumed to be constant for all displacements as long as they are in the linear force regime [85]. This means, for a tightly focused beam, $F_{\mathrm{s}}$ generates a neglectable offset of the trapped particle from the laser focus along the beam axis $[83,85]$ as long as $F_{\mathrm{g}}$ is larger than $F_{\mathrm{S}}[87] . F_{\mathrm{g}}$ or the restoring force is no longer a result of momentum conservation but a lorentzian force resulting from a moving dipole (the bead) in an electro-magnetic field (the beam) [85]. Due to the intensity gradient of the Gauss-shaped laser beam, $F_{\mathrm{g}}$ is acting towards the center of the beam [83] and for small displacements depends linear on the displacement [85]. According to reference [85] $F_{\mathrm{g}}$ can be calculated from:

$$
F_{\mathrm{g}}=\frac{n_{\mathrm{env}} \operatorname{Re}(p) V}{2 c} \cdot \nabla I(x)
$$

for particles with a diameter that is small enough so that the intensity gradient does not 
vary much over the particle size. In this equation $p$ is the polarizability, $V$ the particle volume, $c$ the speed of light and $I$ the intensity of the laser light. The accurate physics behind optical trapping for experiments using particles in the size of the laser wavelength falls somewhere between the two described theories. However, calculations using the better fitting theory of "Mie scattering" are much more complicated [83] and the above described pictures of optical trapping give a good idea about the physics behind this method, even though they are not completely accurate for the bead size used for the experiments of this thesis.

\subsubsection{Calibration of Optical Traps}

To apply OTs as a tool for force spectroscopy in biophysical experiments (like the one performed within the scope of this thesis) it is crucial to be able to measure the force which is currently acting on the sample. Several methods were described to calibrate OTs and some of them are summarized or compared in references [83, 88, 89], among others.

A widely used method to calibrate OTs is based on the assumption that OTs act like Hookean springs. In this case the applied force is directly proportional to the displacement of the bead from the center of the trap following Hook's law:

$$
F=-\kappa_{\text {trap }} \cdot x
$$

where $F$ is the force, $\kappa_{\text {trap }}$ the trap stiffness or spring constant and $x$ the bead displacement from the equilibrium position. One can therefore gain information on both, the particle position and the applied force, by tracking the displacement of the bead from its equilibrium position in the laser focus.

A commonly used method for bead position tracking is back focal plane (BFP) interferometry, where the interference pattern of the forward scattered light and the incident laser beam in the BFP is recorded. Typically this is performed placing a quadrant photodiode (QPD) in the BFP of the OT yielding a very precise measurement of the particle displacement relative to the laser focus in all three dimensions [85].

The calibration of the system is often performed by measuring the thermal fluctuation of a trapped particle and analysis of the power spectrum density (PSD) [85]. The 
displacement $x$ due to a thermal force $F_{\mathrm{t}}$ (in one dimension) can be described by the Langevin equation:

$$
\gamma \cdot \dot{x}(t)+\kappa_{\text {trap }} \cdot x(t)=F_{\text {th }}(t)
$$

with the drag coefficient $\gamma=6 \pi r \eta$ (Stokes law) for a bead of radius $r$ surrounded by a fluid of viscosity $\eta$ [85]. In this system very low Reynolds numbers ( $\operatorname{Re} \approx 10^{-4}-10^{-5}$ ) allow to neglect inertia [90] and the average over time of the random thermal force $\left\langle F_{\mathrm{th}}(t)\right\rangle$ is zero [85]. From the fluctuation dissipation theorem the spectrum of the thermal force of this Langevian equation is described by white noise:

$$
\left\langle F_{\mathrm{th}}(t) F_{\mathrm{th}}(t+\tau)\right\rangle=2 \gamma k_{\mathrm{B}} T \delta(\tau)
$$

with $\delta(\tau)$ being the dirac delta function [85].

The power spectral density $S(\omega)$ is defined as $S(\omega)=|\tilde{x}(\omega)|^{2}$ and is a value that describes how the squared value of the signal is distributed over frequency, e.g. Volt ${ }^{2} /$ Hertz [85]. With the fourier transformation of equation 2.31:

$$
\tilde{F}_{\mathrm{th}}(\omega)=-\gamma \omega i \tilde{x}(\omega)+\alpha \tilde{x}(\omega)
$$

where $\omega=2 \pi f$ is the angular frequency [85] and equation 2.32, the power spectral density is given by:

$$
S(\omega)=\frac{2 \cdot k_{\mathrm{B}} T}{\gamma\left(\omega^{2}+\omega_{c}^{2}\right)}
$$

with $\omega_{c}$ being the roll-off or corner frequency which is related to the trap stiffness by $\omega_{c}=\frac{\kappa_{\text {trap }}}{\gamma}$ [85]. In the power spectrum the corner frequency is the frequency where the almost constant signal (bead is trapped) starts to drop and yields a slope of approximately -2 (freely fluctuating bead) [91]. Fitting equation 2.34 to measured fluctuation data reveals the trap stiffness $\kappa_{\text {trap }}$ from $\omega_{c}$ and $\gamma$ [85].

The power spectrum can also be used as a diagnostic tool for the setup as e.g. misalignment in both the trapping and the detection part do result in a non-Lorentzian power spectrum [92]. The calibration algorithm and the fit function for the power spectrum are under constant development. In Ref. [93], e.g., a detailed analysis of Lorentzian fitting of power spectra was performed and a tool box for precise calibra- 
tion of OTs is presented.

The described calibration method, even though it is widely used has one major drawback: The approximation of a linear dependency between bead displacement and force is only valid for small displacements of the bead from the laser focus [94]. Together with other drawbacks, like its sensitivity on the size of the trapped particle [94], this method requires a frequent recalibration of the instrument and yields accurate force readout only for a small part of the OT potential close to its center.

There is a more direct way to measure the force that is acting on the bead. The basic principle is that the change in momentum flux of the trapping laser due to bead displacement equals directly the force that is applied to the trapped particle [95]. This method was ignored for a long time as it has some tricky instrumental requirements. Most important, it is necessary to collect all the scattered light [96, 97] which is strictly speaking experimentally not feasible [96]. However, Farré and Montes-Usategui showed that for a $1 \mu \mathrm{m}$ polystyrene bead in water about $95 \%$ of the light is scattered in the forward direction even when the bead is far displaced from the center of the trap [98]. In a follow up publication they state that force measurements based on light momentum changes are not restricted to instruments that are fully optimized for this approach but the goodness of the force readout is mainly depending on the percentage of collected light [99]. The light-momentum method is closely related to the above described calibration method and thus a conventional BFP interferometry setup with some changes can be used [99]. Most importantly a position sensitive detector (PSD) must be used instead of a QPD and a high NA (numerical aperture) condenser (preferable NA $\geq 1.3$ ) is required $[97,99]$.

Jahnel et al. directly compared the relationship of voltage and force as well as voltage and displacement. They found the correlation of force and voltage to be linear almost over the full detector size with less than $5 \%$ error. In contrast the assumption of a linear relationship between voltage signal and displacement yielded errors as large as $40 \%$ for larger displacements [94]. Other advantageous of the force calibration by measuring the change in momentum are the independence on the shape and size of the trapped particle, refractive index of the buffer as well as its viscosity and a number of other experiment specific properties [95, 97].

A detailed description on how to build and calibrate an OT that measures force on 
the basis of the change in light momentum is given as a step-by-step instruction in reference [97]. Two ways of calibration are possible to translate the signal on the PSD into a force value. The macroscopic one is based only on setup parameters and yields the detector signal to force conversion factor $\alpha_{\text {sensor: }}$ :

$$
\alpha_{\text {sensor }}=\frac{r_{\mathrm{D}}}{f^{\prime} \cdot \Psi \cdot c}
$$

where $r_{\mathrm{D}}$ is the effective radius of the detector, $f^{\prime}$ is the instruments' focal length and $c$ the speed of light [97]. $\Psi$ represents the responsivity of the setup [97].

Farré et al. also found a "hidden parameter" which is the product of the spring constant times a calibration constant $\beta$ yielding the detector signal to force conversion factor $\alpha_{\text {trap }}$ [99]. Both, $\kappa$ and $\beta$ can be obtained from the power spectrum density as $\kappa=2 \pi \gamma f_{\mathrm{c}}$ and $\beta=\sqrt{\frac{D}{D^{V}}}$, with $D^{V}$ being the fitted diffusion constant. Even though the product of $\kappa$ and $\beta$ is defined in the trap center it was found to be valid for all bead positions [99]. 


\section{References}

1. Szeverenyi, I. et al. The Human Intermediate Filament Database: comprehensive information on a gene family involved in many human diseases. Hum. Mutat. 29, 351-360 (2008).

2. Herrmann, H. \& Aebi, U. Intermediate Filaments. Structure and Assembly. CSH Perspect. Biol. 8 (2016).

3. Herrmann, H., Strelkov, S. V., Burkhard, P. \& Aebi, U. Intermediate Filaments: Primary Determinants of Cell Architecture and Plasticity. J. Clin. Invest. 119, 17721783 (2009).

4. Conway, J. F. \& Parry, D. A. Intermediate Filament Structure. 3. Analysis of Sequence Homologies. Int. J. Biol. Macromol. 10, 79-98 (1988).

5. Herrmann, H. \& Aebi, U. Intermediate Filaments and Their Associates. MultiTalented Structural Elements Specifying Cytoarchitecture and Cytodynamics. Curr. Opin. Cell Biol. 12, 79-90 (2000).

6. Köster, S., Weitz, D. A., Goldman, R. D., Aebi, U. \& Herrmann, H. Intermediate Filament Mechanics in Vitro and in the Cell: From Coiled Coils to Filaments, Fibers and Networks. Curr. Opin. Cell Biol. 32C, 82-91 (2015).

7. Crick, F. H. C. The Packing of Alpha-Helices. Simple Coiled-Coils. Acta Crystallogr. 6, 689-697 (1953).

8. Chernyatina, A. A., Guzenko, D. \& Strelkov, S. V. Intermediate Filament Structure: the Bottom-Up Approach. Curr. Opin. Cell Biol. 32C, 65-72 (2015).

9. Strelkov, S. V. \& Burkhard, P. Analysis of Alpha-Helical Coiled Coils with the Program TWISTER Reveals a Structural Mechanism for Stutter Compensation. J. Struct. Biol. 137, 54-64 (2002).

10. Gruber, M. \& Lupas, A. N. Historical Review. Another 50th Anniversary - New Periodicities in Coiled Coils. Trends Biochem. Sci. 28, 679-685 (2003).

11. Strelkov, S. V. et al. Conserved Segments 1A and 2B of the Intermediate Filament Dimer. Their Atomic Structures and Role in Filament Assembly. EMBO J. 21, 12551266 (2002). 
12. Strelkov, S. V., Schumacher, J., Burkhard, P., Aebi, U. \& Herrmann, H. Crystal Structure of the Human Lamin A Coil 2B Dimer. Implications for the head-to-tail association of nuclear lamins. J. Mol. Biol. 343, 1067-1080 (2004).

13. Lee, C.-H., Kim, M.-S., Chung, B. M., Leahy, D. J. \& Coulombe, P. A. Structural Basis for Heteromeric Assembly and Perinuclear Organization of Keratin Filaments. Nat. Struct. Mol. Biol. 19, 707-715 (2012).

14. Chernyatina, A. A. \& Strelkov, S. V. Stabilization of Vimentin Coil2 Fragment via an Engineered Disulfide. J. Struct. Biol. 177, 46-53 (2012).

15. Block, J. et al. Nonlinear Loading-Rate-Dependent Force Response of Individual Vimentin Intermediate Filaments to Applied Strain. Phys. Rev. Lett. 118, 048101 (2017).

16. Alberts, B. et al. Molecular Biology of the Cell Sixth edition (Garland Science Taylor and Francis Group, New York, NY, 2015).

17. Block, J., Schroeder, V., Pawelzyk, P., Willenbacher, N. \& Koster, S. Physical Properties of Cytoplasmic Intermediate Filaments. Biochim. Biophys. Acta 1853, 30533064 (2015).

18. Hatzfeld, M. \& Franke, W. W. Pair Formation and Promiscuity of Cytokeratins. Formation in Vitro of Heterotypic Complexes and Intermediate-Sized Filaments by Homologous and Heterologous Recombinations of Purified Polypeptides. J. Cell Biol. 101, 1826-1841 (1985).

19. Herrmann, H. et al. Structure and Assembly Properties of the Intermediate Filament Protein Vimentin: The Role of Its Head, Rod and Tail Domains. J. Mol. Biol. 264, 933-953 (1996).

20. Herrmann, H., Häner, M., Brettel, M., Ku, N. O. \& Aebi, U. Characterization of Distinct Early Assembly Units of Different Intermediate Filament Proteins. J. Mol. Biol. 286, 1403-1420 (1999).

21. Steven, A. C., Hainfeld, J. F., Trus, B. L., Wall, J. S. \& Steinert, P. M. The Distribution of Mass in Heteropolymer Intermediate Filaments Assembled in Vitro. Stem Analysis of Vimentin/Desmin and Bovine Epidermal Keratin. J. Biol. Chem. 258, 8323-8329 (1983). 
22. Engel, A., Eichner, R. \& Aebi, U. Polymorphism of Reconstituted Human Epidermal Keratin Filaments. Determination of their Mass-Per-Length and Width by Scanning Transmission Electron Microscopy (STEM). J. Ultra Mol. Struct. R. 90, 323-335 (1985).

23. Colakoglu, G. \& Brown, A. Intermediate Filaments Exchange Subunits Along Their Length and Elongate by End-to-End Annealing. J. Cell Biol. 185, 769-777 (2009).

24. Uchida, A., Colakoglu, G., Wang, L., Monsma, P. C. \& Brown, A. Severing and End-to-End Annealing of Neurofilaments in Neurons. P. Natl. Acad. Sci. USA 110, E2696-705 (2013).

25. Winheim, S. et al. Deconstructing the Late Phase of Vimentin Assembly by Total Internal Reflection Fluorescence Microscopy (TIRFM). PLOS One 6, e19202 (2011).

26. Nöding, B., Herrmann, H. \& Köster, S. Direct Observation of Subunit Exchange along Mature Vimentin Intermediate Filaments. Biophys J 107, 2923-2931 (2014).

27. Vikstrom, K. L., Borisy, G. G. \& Goldman, R. D. Dynamic Aspects of Intermediate Filament Networks in BHK-21 Cells. P. Natl. Acad. Sci. USA 86, 549-553 (1989).

28. Kirmse, R. et al. A Quantitative Kinetic Model for the in Vitro Assembly of Intermediate Filaments from Tetrameric Vimentin. J. Biol. Chem. 282, 18563-18572 (2007).

29. Portet, S. et al. Vimentin Intermediate Filament Formation: In Vitro Measurement and Mathematical Modeling of the Filament Length Distribution during Assembly †. Langmuir 25, 8817-8823 (2009).

30. Sokolova, A. V. et al. Monitoring Intermediate Filament Assembly by Small-Angle X-Ray Scattering Reveals the Molecular Architecture of Assembly Intermediates. P. Natl. Acad. Sci. USA 103, 16206-16211 (2006).

31. Brennich, M. E. et al. Impact of Ion Valency on the Assembly of Vimentin Studied by Quantitative Small Angle X-Ray Scattering. Soft Matter 10, 2059-2068 (2014).

32. Brennich, M. E. et al. Dynamics of Intermediate Filament Assembly Followed in Micro-Flow by Small Angle X-Ray Scattering. Lab Chip 11, 708-716 (2011). 
33. Lichtenstern, T., Mücke, N., Aebi, U., Mauermann, M. \& Herrmann, H. Complex Formation and Kinetics of Filament Assembly Exhibited by the Simple Epithelial Keratins K8 and K18. J. Struct. Biol. 177, 54-62 (2012).

34. Mitchison, T. \& Kirschner, M. Dynamic Instability of Microtubule Growth. Nature 312, 237-242 (1984).

35. Kuhn, J. R. \& Pollard, T. D. Real-Time Measurements of Actin Filament Polymerization by Total Internal Reflection Fluorescence Microscopy. Biophys J 88, 13871402 (2005).

36. Herrmann, H. \& Aebi, U. Intermediate Filaments: Molecular Structure, Assembly Mechanism, and Integration into Functionally Distinct Intracellular Scaffolds. Annu. Rev. Biochem. 73, 749-789 (2004).

37. Charrier, E. E. \& Janmey, P. A. Mechanical Properties of Intermediate Filament Proteins. Method. Enzymol. 568, 35-57 (2016).

38. Shorter, S. A. An Investigation of the Nature of the Elasticity of Fibers. J. Text. I. 15, T207-T229 (1924).

39. Speakman, J. B. 38 - The Intracellular Structure of the Wool Fiber. J. Text. I. 18, T431-T453 (1927).

40. Astbury, W. T. \& Street, A. X-Ray Studies of the Structure of Hair, Wool, and Related Fibres. I. General. Philos. T. R. Soc. Lond. 230, 75-101 (1932).

41. Astbury, W. T. \& Woods, H. J. X-Ray Studies of the Structure of Hair, Wool, and Related Fibres. II. The Molecular Structure and Elastic Properties of Hair Keratin. Philos. T. R. Soc. Lond. 232, 333-394 (1934).

42. Astbury, W. T. \& Sisson, W. A. X-Ray Studies of the Structure of Hair, Wool, and Related Fibres. III. The Configuration of the Keratin Molecule and Its Orientation in the Biological Cell. P. Roy. Soc. Lond. A Mat. 150, 533-551 (1935).

43. Pauling, L. \& Corey, R. B. The Pleated Sheet, A New Layer Configuration of Polypeptide Chains. P. Natl. Acad. Sci. USA 37, 251-256 (1951).

44. Pauling, L. \& Corey, R. B. Compound Helical Configurations of Polypeptide Chains. Structure of Proteins of the alpha-Keratin Type. Nature 171, 59-61 (1953). 
45. Bendit, E. G. A Quantitative X-Ray Diffraction Study of the Alpha-Beta Transformation in Wool Keratin. Text. Res. J. 30, 547-555 (1960).

46. Pinto, N. et al. Self-Assembly Enhances the Strength of Fibers Made from Vimentin Intermediate Filament Proteins. Biomacromolecules 15, 574-581 (2014).

47. Kreplak, L., Bär, H., Leterrier, J. F., Herrmann, H. \& Aebi, U. Exploring the Mechanical Behavior of Single Intermediate Filaments. J. Mol. Biol. 354, 569-577 (2005).

48. Mücke, N. et al. Assessing the Flexibility of Intermediate Filaments by Atomic Force Microscopy. J. Mol. Biol. 335, 1241-1250 (2004).

49. Kiss, B., Karsai, A. \& Kellermayer, M S Z. Nanomechanical Properties of Desmin Intermediate Filaments. J. Struct. Biol. 155, 327-339 (2006).

50. Guzmán, C. et al. Exploring the Mechanical Properties of Single Vimentin Intermediate Filaments by Atomic Force Microscopy. J. Mol. Biol. 360, 623-630 (2006).

51. Fudge, D. S., Gardner, K. H., Forsyth, V. T., Riekel, C. \& Gosline, J. M. The Mechanical Properties of Hydrated Intermediate Filaments: Insights from Hagfish Slime Threads. Biophys J 85, 2015-2027 (2003).

52. Kreplak, L., Herrmann, H. \& Aebi, U. Tensile Properties of Single Desmin Intermediate Filaments. Biophys J 94, 2790-2799 (2008).

53. Qin, Z., Kreplak, L. \& Buehler, M. J. Hierarchical Structure Controls Nanomechanical Properties of Vimentin Intermediate Filaments. PLOS One 4, e7294 (2009).

54. Ramm, B. et al. Sequence-Resolved Free Energy Profiles of Stress Bearing Vimentin Intermediate Filaments. P. Natl. Acad. Sci. USA 111, 11359-11364 (2014).

55. Bertaud, J., Hester, J., Jimenez, D. D. \& Buehler, M. J. Energy Landscape, Structure and Rate Effects on Strength Properties of Alpha-Helical Proteins. J. Phys. Condens. Matter 22, 035102 (2010).

56. Ackbarow, T. \& Buehler, M. J. Alpha-Helical Protein Domains Unify Strength and Robustness Through Hierarchical Nanostructures. Nanotechnology 20, 075103 (2009).

57. Ackbarow, T. \& Buehler, M. J. Superelasticity, Energy Dissipation and Strain Hardening of Vimentin Coiled-Coil Intermediate Filaments: Atomistic and Continuum Studies. J. Mater. Sci. 42, 8771-8787 (2007). 
58. Qin, Z., Kreplak, L. \& Buehler, M. J. Nanomechanical Properties of Vimentin Intermediate Filament Dimers. Nanotechnology 20, 425101 (2009).

59. Qin, Z. \& Buehler, M. J. Structure and Dynamics of Human Vimentin Intermediate Filament Dimer and Tetramer in Explicit and Implicit Solvent Models. J. Mol. Model. 17, 37-48 (2011).

60. Chou, C.-C. \& Buehler, M. J. Structure and Mechanical Properties of Human Trichocyte Keratin Intermediate Filament Protein. Biomacromolecules 13, 35223532 (2012).

61. Chou, C.-C., Lepore, E., Antonaci, P., Pugno, N. \& Buehler, M. J. Mechanics of Trichocyte Alpha-Keratin Fibers. Experiment, Theory, and Simulation. J. Mater. Res. 30, 26-35 (2015).

62. Antunes, E., Cruz, C. F., Azoia, N. G. \& Cavaco-Paulo, A. Insights on the Mechanical Behavior of Keratin Fibrils. Int. J. Biol. Macromol. 89, 477-483 (2016).

63. Fudge, D. et al. The Intermediate Filament Network in Cultured Human Keratinocytes is Remarkably Extensible and Resilient. PLOS One 3, e2327 (2008).

64. Ramms, L. et al. Keratins as the Main Component for the Mechanical Integrity of Keratinocytes. P. Natl. Acad. Sci. USA 110, 18513-18518 (2013).

65. Seltmann, K., Fritsch, A. W., Käs, J. A. \& Magin, T. M. Keratins Significantly Contribute to Cell Stiffness and Impact Invasive Behavior. P. Natl. Acad. Sci. USA 110, 18507-18512 (2013).

66. Seltmann, K. et al. Keratins Mediate Localization of Hemidesmosomes and Repress Cell Motility. J. Invest. Dermatol. 133, 181-190 (2013).

67. Brown, M. J., Hallam, J. A., Colucci-Guyon, E. \& Shaw, S. Rigidity of Circulating Lymphocytes Is Primarily Conferred by Vimentin Intermediate Filaments. J. Immunol. 166, 6640-6646 (2001).

68. Wang, N. \& Stamenovic, D. Contribution of Intermediate Filaments to Cell Stiffness, Stiffening, and Growth. Am. J. Physiol. - Cell Ph. 279, C188-C194 (2000).

69. Guo, M. et al. The Role of Vimentin Intermediate Filaments in Cortical and Cytoplasmic Mechanics. Biophys J 105, 1562-1568 (2013). 
70. Mendez, M. G., Kojima, S.-I. \& Goldman, R. D. Vimentin Induces Changes in Cell Shape, Motility, and Adhesion during the Epithelial to Mesenchymal Transition. Faseb J. 24, 1838-1851 (2010).

71. Mendez, M. G., Restle, D. \& Janmey, P. A. Vimentin Enhances Cell Elastic Behavior and Protects Against Compressive Stress. Biophys J 107, 314-323 (2014).

72. van der Maarel, J. R. C. Introduction to Biopolymer Physics (World Scientific, Singapore, 2008).

73. Andrews, S. S. Methods for Modeling Cytoskeletal and DNA Filaments. Phys. Biol. 11, 011001 (2014).

74. Kumar, S. \& Li, M. S. Biomolecules Under Mechanical Force. Phys. Rep. 486, 1-74 (2010).

75. Bustamante, C., Marko, J. F., Siggia, E. D. \& Smith, S. Entropic Elasticity of LambdaPhage DNA. Science 265, 1599-1600 (1994).

76. Rief, M. Elastically Coupled Two-Level Systems as a Model for Biopolymer Extensibility. Phys. Rev. Lett. 81, 4764-4767 (1998).

77. Burte, H. \& Halsey, G. A New Theory of Non-Linear Viscous Elasticity. Text. Res. J. 17, 465-476 (1947).

78. Bell, G. Models for the Specific Adhesion of Cells to Cells. Science 200, 618-627 (1978).

79. Evans, E. \& Ritchie, K. Dynamic Strength of Molecular Adhesion Bonds. Biophys J 72, 1541-1555 (1997).

80. Friddle, R. W., Noy, A. \& de Yoreo, J. J. Interpreting the Widespread Nonlinear Force Spectra of Intermolecular Bonds. P. Natl. Acad. Sci. USA 109, 13573-13578 (2012).

81. Ashkin, A. Acceleration and Trapping of Particles by Radiation Pressure. Phys. Rev. Lett. 24, 156-159 (1970).

82. Ashkin, A. History of Optical Trapping and Manipulation of Small-Neutral Particle, Atoms, and Molecules. IEEE J. Sel. Top. Quant. 6, 841-856 (2000).

83. Shaevitz, J. W. A Practical Guide to Optical Trapping. Technical Report, Princeton University (2006). 
84. Ashkin, A., Dziedzic, J. M., Bjorkholm, J. E. \& Chu, S. Observation of a Single-Beam Gradient Force Optical Trap for Dielectric Particles. Opt. Lett. 11, 288-290 (1986).

85. Koch, M. D. \& Shaevitz, J. W. Introduction to Optical Tweezers. Methods in Molecular Biology 1486, 3-24 (2017).

86. Ashkin, A. Forces of a Single-Beam Gradient Laser Trap on a Dielectric Sphere in the Ray Optics Regime. Biophys J 61, 569-582 (1992).

87. Fundamental Tests of Physics with Optically Trapped Microspheres (ed Li, T.) (Springer New York, New York, NY, 2013). ISBN: 978-1-4614-6030-5. doi:10.1007/ 978-1-4614-6031-2.

88. Sarshar, M., Wong, W. T. \& Anvari, B. Comparative Study of Methods to Calibrate the Stiffness of a Single-Beam Gradient-Force Optical Tweezers over Various Laser Trapping Powers. J. Biomed. Optics 19, 115001 (2014).

89. Neuman, K. C. \& Block, S. M. Optical Trapping. Rev. Sci. Instrum. 75, 2787-2809 (2004).

90. Purcell, E. M. Life at Low Reynolds Number. Am. J. Phys. 45, $49-64$ (1977).

91. Gittes, F. \& Schmidt, C. F. Chapter 8 Signals and Noise in Micromechanical Measurements. Methods in Cell Biology 55, 129-156 (1997).

92. Neuman, K. C. \& Nagy, A. Single-Molecule Force Spectroscopy. Optical Tweezers, Magnetic Tweezers and Atomic Force Microscopy. Nat. Methods 5, 491-505 (2008).

93. Berg-Sørensen, K. \& Flyvbjerg, H. Power Spectrum Analysis for Optical Tweezers. Rev. Sci. Instrum. 75, 594 (2004).

94. Jahnel, M., Behrndt, M., Jannasch, A., Schäffer, E. \& Grill, S. W. Measuring the Complete Force Field of an Optical Trap. Opt. Lett. 36, 1260-1262 (2011).

95. Smith, S. B., Cui, Y. \& Bustamante, C. Optical-Trap Force Transducer that Operates by Direct Measurement of Light Momentum. Method. Enzymol. 361, 134-162 (2003).

96. Thalhammer, G., Obmascher, L. \& Ritsch-Marte, M. Direct Measurement of Axial Optical Forces. Opt. Express 23, 6112-6129 (2015). 
97. Farre, A., Marsa, F. \& Montes-Usategui, M. Beyond the Hookean Spring Model. Direct Measurement of Optical Forces Through Light Momentum Changes. Methods in Molecular Biology 1486, 41-76 (2017).

98. Farre, A. \& Montes-Usategui, M. A Force Detection Technique for Single-Beam Optical Traps Based on Direct Measurement of Light Momentum Changes. Opt. Express 18, 11955-11968 (2010).

99. Farre, A., Marsa, F. \& Montes-Usategui, M. Optimized Back-Focal-Plane Interferometry Directly Measures Forces of Optically Trapped Particles. Opt. Express 20, 12270-12291 (2012). 



\section{Chapter 3}

\section{Materials and Methods}

Parts of this chapter are, either in full length or in shorter versions, also included in the publication [1], which is reprinted as Chapter 4 and in the publication [2] reprinted as Chapter5.

Full names of chemicals and proteins are listed in Tab. 3.1 in the end of this chapter. If not stated differently, solutions and buffers were prepared with MilliQ-water.

All experiments described in this work were performed with a genetically modified version of human vimentin. The cysteine at amino acid position 328 was replaced by alanine and two glycine and one cysteine were incorporated at the C-terminal end of the protein. The plasmid, containing the protein of interest, was kindly provided by Harald Herrmann (DKFZ, Heidelberg, Germany).

\subsection{Vimentin expression and purification}

Vimentin was expressed recombinantly in Escherichia coli (E. coli) T61 cells (Zymo Research, Irvine, CA, USA, \#3017). Protein preparation was performed by the technician Susanne Bauch following a protocol adapted from [3]. 
$100 \mu \mathrm{l}$ E. coli solution was thawed, mixed with $1 \mu \mathrm{l}$ plasmid solution (about $1 \mu \mathrm{g} / \mu \mathrm{L}$, diluted 1:100) and incubated on ice for 5 minutes. Afterwards, $50 \mu \mathrm{l}$ of E. coli solution were plated on a LB agar plate, containing ampicillin (AMP), and incubated at $37^{\circ} \mathrm{C}$ over night. A single bacteria colony was transferred into $50 \mathrm{~mL}$ autoclaved TB medium $(47.6 \mathrm{~g} / \mathrm{L})$, containing glycerol $(10-11 \mathrm{~g} / \mathrm{L})$ and ampicillin $(0.1 \mathrm{~g} / \mathrm{L})$, incubated for $6 \mathrm{~h}$ at $37^{\circ} \mathrm{C}$ and 150 rounds per minute (rpm). For the next step, cultured bacteria solution was transferred to $1 \mathrm{~L}$ fresh TB medium containing $0.1 \mathrm{~g} / \mathrm{L}$ AMP and incubated at $37^{\circ} \mathrm{C}$ and 150 rpm over night.

For protein purification bacteria were pelleted using a Beckman centrifuge at $5000 \mathrm{~g}$ for $15 \mathrm{~min}$ at $4{ }^{\circ} \mathrm{C}$ (Beckmann Coulter Avanti J-26 XP, rotor JS5.3, Krefeld, Germany). The following steps were all performed using cooled instruments or on ice. The pellet was homogenized with $16 \mathrm{~mL} 50 \mathrm{mM}$ TRIS buffer, containing $25 \%$ saccarose (FIXME), $1 \mathrm{mM}$ EDTA and $10 \mathrm{~g} / \mathrm{L}$ lysozym, after transferring to a cooled douncer. The mixture was incubated on ice for $30 \mathrm{~min}$.

$800 \mu \mathrm{l} 1 \mathrm{M} \mathrm{MgCl}_{2}, 80 \mu \mathrm{l}$ DNAse1 (50 g/L in $10 \mathrm{mM}$ TRIS buffer, pH 7.5, containing $100 \mathrm{mM} \mathrm{NaCl}), 80 \mu \mathrm{l}$ RNAse A (10 g/L in $100 \mathrm{mM}$ TRIS buffer, pH 7.5), $800 \mu$ l saturated PMSF and $1.6 \mathrm{~mL} 10 \%$ NP40 were added and homogenized several times. $40 \mathrm{~mL} 20 \mathrm{mM}$ TRIS buffer, pH 7.5, containing $200 \mathrm{mM} \mathrm{NaCl}, 2$ mM EDTA, $1 \%$ NP40 and $1 \%$ DOC was mixed with $800 \mu \mathrm{LTT}, 1.2 \mathrm{~mL}$ PMSF and $0.4 \mathrm{~mL} 50 \mathrm{mM}$ Pefabloc SC and added to the douncer. After a $10 \mathrm{~min}$ incubation step, the mixture was filled to Nalgene centrifuge bottles (250 mL PPCO-centrifuge bottles with sealing closure, Fisher Scientific GmbH (part of Thermo Fisher Scientific), Schwerte, Germany) and pelleted using a Beckman centrifuge (Beckman Coulter Avanti, J-26 XP, rotor JLA 16.250) at $4{ }^{\circ} \mathrm{C}$ and 10,000 $\mathrm{g}$ for $25 \mathrm{~min}$.

The supernatant was discarded, washing buffer (80 mL $10 \mathrm{mM}$ TRIS buffer, pH 8.0, with $5 \mathrm{mM}$ EDTA and $1 \%$ Triton-X100, $80 \mu \mathrm{l} 1 \mathrm{M}$ DTT, $400 \mu \mathrm{l}$ PMSF, $200 \mu \mathrm{l} 50 \mathrm{mM}$ Pefabloc SC) was added and the pellet was washed by homogenization in the douncer. After 10 min incubation on ice, centrifugation is performed as before. The supernatant was discarded. $80 \mathrm{~mL}$ washing buffer, additionally containing $1.5 \mathrm{M} \mathrm{KCl}$, was added and everything was mixed by homogenization in a douncer. The mixture was incubated on ice for $30 \mathrm{~min}$ and centrifuged as before. As a third washing step, the first washing step was exactly repeated. The buffer for washing step four consisted of $80 \mathrm{~mL}$ TRIS 
buffer, pH 8.0, with 0.1 mM EDTA, $80 \mu \mathrm{l}$ DTT, $200 \mu \mathrm{l} 50 \mathrm{mM}$ Pefabloc SC and $400 \mu \mathrm{l}$ PMSF. Following the same procedure as before, the pellet was washed, incubated on ice for $10 \mathrm{~min}$, centrifuged and the supernatant discarded.

The pellet was transferred to a douncer, warmed to room temperature and, to yield a high protein concentration, dissolved in as little volume of $9.5 \mathrm{M}$ urea solution (7.125 mL 9.5 M urea, $75 \mu l 1$ M TRIS, pH 7.5, $75 \mu \mathrm{l}$ 0.5 M EDTA, $75 \mu \mathrm{l}$ PMFS and $75 \mu \mathrm{l} 1 \mathrm{M}$ DTT) as possible. The mixture was centrifuged in a Beckman ultracentrifuge (Beckamn Coulter Optima L90K, rotor Ti70, Krefeld, Germany) at $20^{\circ} \mathrm{C}$ and 100,000 $\mathrm{g}$ for $60 \mathrm{~min}$. The supernatant - containing the vimentin - was transferred to a Falcon tube and MAC was added until a final concentration of $10 \mathrm{mM}$ was reached.

For the anion exchange chromatography a DEAE column (BioRad, Hercules, CA, USA, \#737-1522, inner diameter $15 \mathrm{~mm}$, length $200 \mathrm{~mm}$, volume $35 \mathrm{~mL}$ ) was used. $10 \mathrm{~mL}$ column material (DEAE sepharose) was mixed with $10 \mathrm{~mL}$ column buffer (400 $\mathrm{mL}$ urea (10 M), $50 \mathrm{~mL} 50 \mathrm{mM}$ TRIS (pH 8.0), containing EDTA (10 mM) and EGTA (1 $\mathrm{mM}$ ), filled to $500 \mathrm{~mL}$ after mixing). Column material was allowed to settle down according to the supplier instructions. A $\mathrm{KCl}$ gradient was used to eluate the protein. A gradient mixer ( 2 x $50 \mathrm{~mL}$, Schütt 24, Göttingen, Germany) was filled with $40 \mathrm{~mL}$ column buffer in the near chamber and $40 \mathrm{~mL}$ column buffer complemented by $0.896 \mathrm{~g}$ $\mathrm{KCl}$ in the front chamber. The protein solution was added on the column. After the sample had entered the column bed, it was washed with two column volumes of column buffer (slow flow velocity). Sample collection began when the collected volume had reached the dead volume of the column. Therefore, the salt gradient was started and the column volume was kept constant by regulation of inflow and outflow. The sample volume was collected in $1.5 \mathrm{~mL}$ Eppendorf cups and protein concentration was monitored by UV-Vis spectrometry (NanoDrop ND-1000, ThermoScientific Technologies, Inc., Wilmington, USA).

As an additional quality control, an $8 \%$ SDS-gel was performed prior to the final purification step. Samples collected during the purification process and samples taken from protein fractions after DEAE column were mixed with sample buffer - $10 \mu \mathrm{l}$ sample (in case of high protein concentration $5 \mu$ protein solution and $5 \mu$ l MilliQ water), $12.5 \mu \mathrm{l}$ sample buffer, $22.5 \mathrm{ml}$ MilliQ water and $5 \mu \mathrm{l}$ M DTT. Prepared samples were denatured at $70^{\circ} \mathrm{C}$ for $10 \mathrm{~min}$ and loaded on the gel, a protein marker was added to 
the first well. The gel was placed in an electrophoresis system (VWR/CBS, CBDCX-700, Darmstadt, Germany) and performed for $75 \mathrm{~min}$ at $100 \mathrm{~mA}$ (for two gels $200 \mathrm{~mA}$ ). Afterwards, the gel was stained using InstantBlue ${ }^{\mathrm{TM}}$ for 15 to $60 \mathrm{~min}$.

The fractions with the highest protein concentration after DEAE column were pooled for a cation exchange chromatography. This second chromatography was performed in analogy to the anion exchange chromatography with the following changes. The column was filled with CM-Sepharose as column material and $40 \mathrm{~mL}$ column buffer complemented by $1.792 \mathrm{~g} \mathrm{KCl}$, were filled in the front chamber. After the sample was added to the column the salt gradient was started directly. The protein concentration of the eluate was monitored by measuring the absorption at $280 \mathrm{~nm}$ using the NanoDrop. Fractions with a high protein concentration were pooled. Lastly, MAC was added until a final concentration of $10 \mathrm{mM}$ was reached. Aliquots of the protein solution were stored at $-80^{\circ} \mathrm{C}$. The protein should, according to Harald Herrmann (DKFZ, Heidelberg, Germany), not be used for longer than three years after production. Thawing and re-freezing should be avoided as much as possible.

\subsection{Labeling of Vimentin}

For fluorescence microscopy vimentin had to be labeled with a fluorescent dye, in this case ATTO647N. OT stretching measurements of vimentin requested coupling to a bead. In most of the experiments a biotin streptavidin reaction, with biotin labeled vimentin, was used for coupling. The very specific sulfhydryl-reactive crosslinker chemistry was used to ensure that only the cysteine at the tail domain was labeled and the interaction of the label with the $\alpha$-helical rod domain and the hierarchical formation of the vimentin filament was minimal.

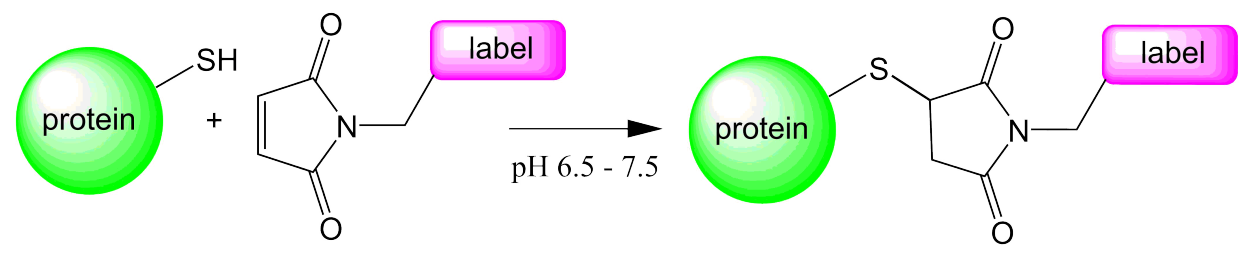

Figure 3.1: Reaction scheme of the maleimide cysteine coupling reaction. 


\subsubsection{Labeling with Fluorescent Dyes}

The experimental procedure for vimentin labeling via maleimide chemistry was adopted from references [4, 5]. $500 \mu \mathrm{l}$ vimentin (concentration approx. $2.7 \mathrm{~g} / \mathrm{L}$ ) was placed into a $50 \mathrm{kDa}$ dialysis tubing (Spectra/Por®7, dialysis membrane made of regenerated cellulose, MWCO 50000, E883.1, Carl-Roth GmbH, Karlsruhe, Germany) and dialyzed into labeling buffer $\left(50 \mathrm{mM} \mathrm{NaH}_{2} \mathrm{PO}_{4}, 50 \mathrm{mM} \mathrm{Na}_{2} \mathrm{HPO}_{4}, 5 \mathrm{M}\right.$ urea, $\mathrm{pH}$ 7.0) at $10{ }^{\circ} \mathrm{C}$ over night.

After dialysis, vimentin solution was placed in an $1.5 \mathrm{~mL}$ reaction tube and the concentration was adjusted to approximately $1 \mathrm{~g} / \mathrm{L}$ by dilution with labeling buffer (monitored by UV-vis spectroscopy). $1 \mathrm{~mL}$ of this solution was filled into in a new $1.5 \mathrm{~mL}$ reaction tube. $20 \mu \mathrm{l}$ of $10 \mathrm{mM}$ ATTO647N-maleimide, dissolved in DMSO, were added stepwise in $5 \mu$ l portions. Each addition of ATTO647N was followed by an incubation step of $5 \mathrm{~min}$, where the tube was placed on a shaker. Subsequently, the sample was incubated for $120 \mathrm{~min}$ at room temperature to complete the labeling reaction. Lastly $100 \mu \mathrm{l} \mathrm{M} \mathrm{L}$-cysteine were added and the mixture was incubating for another $1 \mathrm{~h}$ at room temperature to ensure that the remaining maleimide reacted and did not bind unspecific to vimentin.

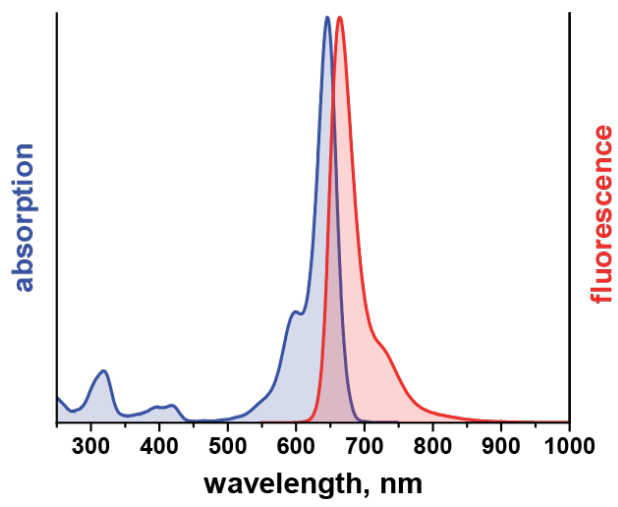

Figure 3.2: Excitation and emission spectrum of ATTO647N. Ideal wavelength for excitation is $\lambda_{\text {ex }}=$ $646 \mathrm{~nm}$ and the maximum emission wavelength $\lambda_{e m}=664 \mathrm{~nm}$. Image from https://www.attotec.com/attotecshop/product_info.php?info=p114_atto-647n.html. 
Free dye and labeled protein were separated by size-exclusion chromatography, using a $300 \mathrm{~mm}$ Bio-Gel P polyacrylamide gel column with a bed volume of $24 \mathrm{~mL}$. Column material (BioGel P30) was hydrated in labeling buffer at room temperature over night and the column was prepared as recommended in the instructions manual. The protein-label solution was placed on top of the column and was allowed to enter the bed completely. Labeling buffer was used to flush the column and fractions with labeled vimentin were collected in about $250 \mu$ l aliquots. Protein and dye concentration were monitored by UV-vis spectroscopy. Fractions with the highest protein concentration were pooled. The final protein and dye concentrations was measured (UV-vis spectroscopy) and the labeling ratio was calculated. Lastly the labeled vimentin was dialyzed into storage buffer and stored at $-80^{\circ} \mathrm{C}$ in aliquots of about 50 to $100 \mu$ l. Different from unlabeled vimentin, storage buffer for labeled vimentin only contained $2 \mathrm{mM}$ phosphate buffer, pH 7.5 and $8 \mathrm{M}$ urea. Aliquots were used up within two years.

\subsubsection{Labeling with Biotin}

The procedure for labeling with maleimide-biotin was mostly identical to the labeling with fluorescent dyes except for the size exclusion chromatography. Separation of labeled vimentin and free maleimide-biotin was performed using three prepacked and disposable PD MidiTrap G-25 columns containing 3.5 mL Sephadex G-25 resin (GE Healthcare Europe GmbH, Freiburg, Germany) which were equilibrated with labeling buffer. After labeling and addition of cysteine, the sample was placed on the first column and eluted by adding $1.5 \mathrm{~mL}$ labeling buffer, yielding one fraction of $1.5 \mathrm{~mL}$. Half of this fraction was added to the other two columns, each. To completely fill the columns, $250 \mathrm{~mL}$ labeling buffer was added to each of them after the protein solution had entered the bed. Subsequently biotin-labeled vimentin was eluted using $1.5 \mathrm{~mL}$ labeling buffer on both columns. The collected flow-through was pooled and, after dialysis into storage buffer (as dye-labeled vimentin), aliquoted into $100 \mu$ l portions for storage at $-80^{\circ} \mathrm{C}$. Different from dye-labeled vimentin it was not straight forward to measure the protein and biotin concentration in the sample after labeling, even though a UV tracker biotin was used. Therefore, biotin-labeled vimentin was only added to an amount that ensured that the total amount of biotin-labeled vimentin in the sample did not exceed $10 \%$. The volumes that had to be mixed were calculated due to the vi- 
mentin concentration of the unlabeled vimentin and the vimentin prior to the labeling reaction. Because of the two MidiTRap column steps the sample is diluted by a factor of three and the vimentin concentration not higher than $0.3 \mathrm{~g} / \mathrm{L}$ anymore.

\subsection{Vimentin Reconstitution and Assembly}

For optical trap experiments, unlabeled, ATTO647N-labeled and biotin-labeled vimentin was mixed to a final content of 2.5 to $5 \%$ ATTO647N labeled and about $10 \%$ biotin-labeled vimentin in the assembled filament. For reconstitution vimentin was dialyzed step-wise from denaturing $8 \mathrm{M}$ urea buffer into phosphate buffer $(2 \mathrm{mM}$ $\mathrm{NaH}_{2} \mathrm{PO}_{4}, 2 \mathrm{mM} \mathrm{Na}_{2} \mathrm{HPO}_{4}, \mathrm{pH}$ 7.5). Therefore, the mixture was filled into $50 \mathrm{kDa}$ dyalisis tubing and, for $30 \mathrm{~min}$ each, placed into a beaker containing 8, 6, 4, 2, 1 and $0 \mathrm{M}$ urea in phosphate buffer. For a final cleaning step the sample was placed into a fresh beaker containing pure phosphate buffer and was dialyzed either for two hours at room temperature or at $10^{\circ} \mathrm{C}$ over night. Prior to assembly, the sample was diluted to a protein concentration of $0.2 \mathrm{~g} / \mathrm{L}$ adding pure phosphate buffer. Protein concentration was monitored using UV-vis spectroscopy. The sample was aliquoted into portions of at least $200 \mu \mathrm{l}$ and stored, protected from light, at $4{ }^{\circ} \mathrm{C}$ for a maximum of one week.

Assembly of vimentin is ion-driven, in this case $100 \mathrm{mM}$ potassium chloride $(\mathrm{KCl})$ was used to initiate filament formation. For a slow assembly, that leads to more homogeneous filaments, the sample was filled into $50 \mathrm{kDa}$ dialysis tubing and placed in a beaker with assembly buffer $\left(2 \mathrm{mM} \mathrm{NaH}_{2} \mathrm{PO}_{4}, 2 \mathrm{mM} \mathrm{Na}_{2} \mathrm{HPO}_{4}, 100 \mathrm{mM} \mathrm{KCl}, \mathrm{pH}\right.$ 7.5), adjusted to $37^{\circ} \mathrm{C}$. For a kick-start assembly vimentin with a concentration of $0.4 \mathrm{~g} / \mathrm{L}$ was mixed 1:1 with assembly buffer containing $200 \mathrm{mM} \mathrm{KCl}$, so the final concentrations for the kick-start assembly was $0.2 \mathrm{~g} / \mathrm{L}$ vimentin and $100 \mathrm{mM} \mathrm{KCl}$, too. Filament formation was in both cases performed over night at $37^{\circ} \mathrm{C}$. Assembled filaments were kept at $4{ }^{\circ} \mathrm{C}$. 


\subsection{Maleimide-functionalization of Beads}

Beads were functionalized with a maleimide-group according to a protocol published by Janissen et al. in 2014[6]. Carboxylated polystyrene beads (Kisker biotech $\mathrm{GmbH}$ \& Co. KG, Steinfurt, Germany - German reseller of Spherotech -, PPs-4.2COOH, 4.0$4-4 \mu \mathrm{m}, 5 \%(\mathrm{w} / \mathrm{v}))$ where placed on a tilting mixer for $30 \mathrm{~min}$ to avoid aggregates. $100 \mu \mathrm{l}$ bead solution were placed in a $1 \mathrm{~mL}$ Eppendorf tube and centrifuged (Eppendorf centrifuge 5417R, FA45-24-11) until 10000 rcf were reached. The supernatant was discarded and the beads were washed twice with $1 \mathrm{~mL}$ of $100 \mathrm{mM}$ MES buffer, $\mathrm{pH} 4.7$, for $10 \mathrm{~min}$ on a pulsing vortex mixer at $1200 \mathrm{rpm}$. Between and after the washing steps the beads were centrifuged down and the supernatant was discarded.

For functionalization, beads were resuspended in $100 \mu \mathrm{l}$ of $100 \mathrm{mM}$ MES buffer and another $100 \mu \mathrm{l}$ MES buffer containing $4 \mathrm{mg}$ maleimide-PEG- $\mathrm{NH}_{2}$ (kindly provided by Nynke Dekker group, Delft University of Technology, Netherlands) were added. $8 \mathrm{mg}$ $\mathrm{NH}_{2}$-PEG-OH and $20 \mathrm{mg}$ EDC were added. The mixture was thoroughly mixed for $2 \mathrm{~min}$ by vortexing and sonicated for $2 \mathrm{~h}$ with mixing for $30 \mathrm{~s}$ every $20 \mathrm{~min}$.

Finally, the beads were washed with $1 \mathrm{~mL} 1$ xPBS containing $2 \%$ BSA three times for $10 \mathrm{~min}$ on a shaker at $1200 \mathrm{rpm}$. After each washing step, beads were centrifuged down and the supernatant was discarded. Functionalized beads were resuspended and stored in $1 \mathrm{~mL} 1$ xPBS containing $2 \%$ BSA, pH 7.4 at $4{ }^{\circ} \mathrm{C}$. The protocol of Janissen et al. recommends to use the beads no longer than 30 days[6].

\subsection{Optical Tweezers Experiments}

Except four weeks during which the OT setup "C-Trap" (LUMICKS, Amsterdam, The Netherlands) was installed in Göttingen, all experiments were carried out in the labs of E. J. G. Petermann and G. J. L. Wuite (Department of Physics and Astronomy and LaserLab, Vrije Universiteit Amsterdam, 1081 HV Amsterdam, The Netherlands). Experiments in Amsterdam were performed with technical support by either Andrea Candelli, Jordi Cabanas-Danes or Margherita Marchetti. Due to the traveling, one set of measurements in Amsterdam always lasted from Monday morning to Friday afternoon. 


\subsubsection{Setup}

Most of the measurements described in this thesis, were performed on a LUMICKS CTrap. The first two weeks of measurements were performed on a lab-built instrument (lab of G. Wuite and E. Peterman). Both instruments use comparable dual-optical traps and equal microfluidic chips in the following called flow cells. The lab-built instrument employed wide-field fluorescence microscopy, while the C-Trap employed confocal fluorescence microscopy. A detailed description of the lab-built setup can be found in references $[7,8]$. In contrast to the description in the references, the NIR-laser (1064 nm) for trapping had a power of $12 \mathrm{~W}$. The trapping laser incorporated in the C-Trap was a 20 W NIR (1064 nm) laser. The laser beam was split, in order to gain two independently controlled traps. Due to their different properties, the two traps were used for different purposes. Trap 1 was highly position stable and therefore used for force detection, while trap 2 was smoothly movable and used for bead manipulation, as indicated in Fig. 3.3.

For force detection, the transmitted trapping light was collected via a high NA oil immersion condensor and imaged using a position-sensitive detector, which for the CTrap had a dimension of $10 \times 10 \mathrm{~mm}$ and a rise time of $4 \mu \mathrm{s}$. Trap steering was piezo based. The sample-holder was a combination of a micro- and a nanostage, where the microfluidics system was incorporated. Bead tracking was performed by acquiring bright field images of the LED illuminated trapped beads using a CCD camera. The control software for both setups was TWOM, a LabView based software that was originally developed in the lab of Wuite and Peterman. Fig. 3.4 shows the key features of the setup (A) and the flow cell (B). The software was constantly improved by the members of the Wuite and Peterman lab (especially by Onno Broekmans) during the duration of this thesis. However, the operation control did not change much.

\subsubsection{Calibration of Optical Traps}

The force calibration of the optical trap was performed using a TWOM software internal calibration tool. This tool used the power spectrum analysis of the position of the trapped bead, which is based on the correlation between the frequency component of the particle motion and the trap stiffness as described in Section 2.4. 


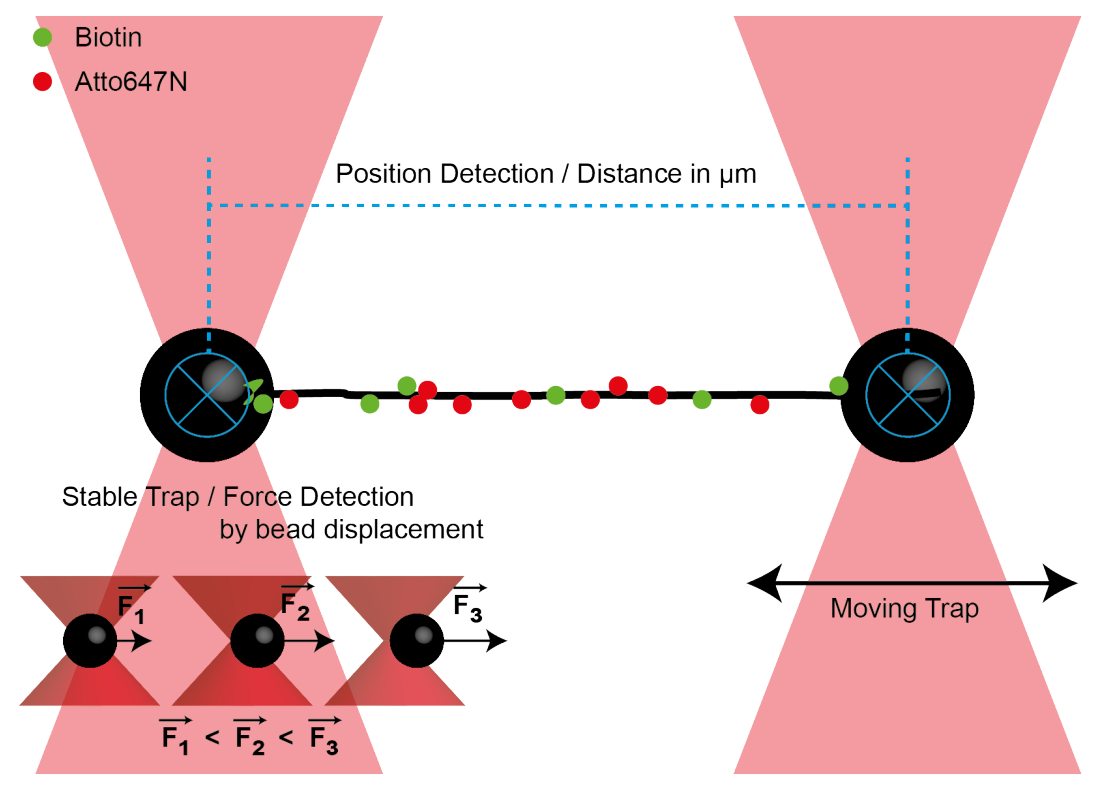

Figure 3.3: Illustration of a dual optical trap, showing two traps, including trapped beads and a labeled vimentin filament. The left trap (trap one) is the "stable" trap which is used for force measurements. The right trap (trap two) is used to actively manipulate the filament in between the two beads. The filament length is quantified by tracking the distance between the centers of the two beads. The force is measured by monitoring the displacement of the bead from the center of the optical trap.

\subsubsection{Sample Preparation}

Four to three days before starting the measurement series, vimentin was dialyzed into phosphate buffer as described above. After dialysis, the protein concentration was adjusted to $0.2 \mathrm{~g} / \mathrm{L}$ and subsequently the sample aliquoted into five portions - one for every day of measurements. For measurements in Amsterdam, the sample for Monday was assembled over night from Saturday to Sunday in Göttingen, while for the other four days and for most of the measurements in Göttingen vimentin was assembled freshly over night. In the beginning, assembled vimentin was used for more than one day. This procedure was optimized during the work on this thesis. Assembled protein was always kept in the fridge or on ice until it was filled into the setup. 


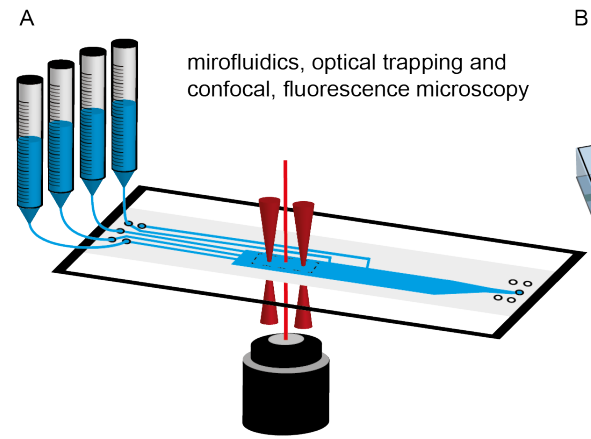

B

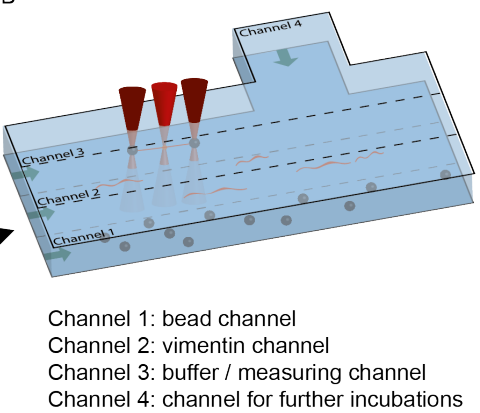

Figure 3.4: (A) Key features of the experimental setup. Solutions are filled into syringes and flushed into the microfluidic chip by air pressure. Fluorescence microscopy is used for visualization. Dual optical tweezers are used to manipulate the filaments and measure the responding force. (B) Schematic $3 D$ view of the flow-cell. All solutions are flushed into the channels, which, due to laminar flow, do not mix. channel 1: beads in assembly buffer, channel 2: vimentin in assembly buffer, channel 3: as sembly buffer, used for actual measurements, channel 4: assembly buffer, containing manipulating chemicals like glutaraldehyde.

\subsubsection{Measurement Preparation and Basic Experimental Procedure}

The microfluidic system, visualized in Fig. 3.4 is one of the key features of the setup. Starting from four individual channels all needed solutions end up in one big channel. Due to laminar flow, the solutions do not mix in the region where the measurements are situated. With this geometry it is possible to spatially separate the different experimental steps within one microfluidic chip. The flow is air-pressure driven. The solutions are filled to the syringes (syringe 1: beads in assembly buffer, syringe 2: vimentin in assembly buffer, syringe 3: pure assembly buffer, syringe 4: assembly buffer, containing manipulating chemicals like glutaraldehyde), corresponding to the four channels.

For each data set a fresh pair of beads was captured in channel 1, and moved into the buffer channel (channel3) by moving the flow cell with respect to the optical tweezers. While moving to channel3, the vimentin channel (channel2) was passed and eventually vimentin filaments bound to the beads. Due to the flow, vimentin filaments, that had bound to the beads, were stretched out along the channel and by bringing the second bead (bead in trap 2) closer to the first bead (bead in trap 1) one filament eventually bound to the second bead, too. Other filaments, that had bound to one of the 


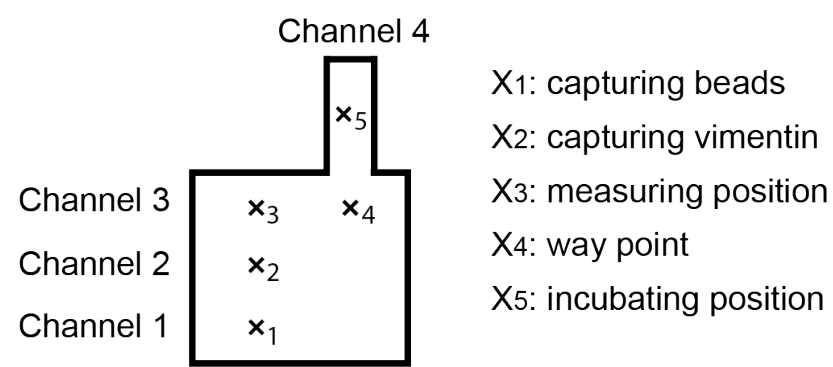

Figure 3.5: Experimental Steps Diagram. Defined positions within the flow cell was used for the different steps of each experiment. At position $X_{1}$ beads were captured, at position $X_{2}$ beads were incubated until vimentin had bound to them. The actual experiments was conducted at position $X_{3}$. For incubation with glutaraldehyde, position $X_{5}$ was used. In order to get to position $X_{5}$ the software needed to move via the way-point $X_{4}$.

beads, probably wrapped around the beads after the flow was stopped for measurements. Fig. 3.5 illustrates the region of the flow cell where the experiments took place. $\mathrm{X}_{1}$ to $\mathrm{X}_{5}$ denote the positions where the traps were stopped for bead and filament capturing, incubation and measurements. Fluorescence microscopy was used to ensure that only one filament was measured per experiment (Fig. 3.6). Cross-diffusion, due to the stopped flow and the therefore broken laminar conditions, would theoretically be possible but is irrelevant for particles of the size of the used beads and the vimentin filaments. The diffusion constant of the bead is on the order of $10^{-} 13 \frac{\mathrm{m}^{2}}{\mathrm{~s}}$ and its sink velocity is about $20 \frac{\mu \mathrm{m}}{\mathrm{s}}$. This means that the beads are already on the bottom of the flow cell after about $2 \mathrm{~s}$. The vimentin concentration is very low $\left(2 \cdot 10^{-4} \frac{\mathrm{g}}{\mathrm{L}}\right)$, the diffusion constant in the same range as the one of the beads and imaging (fluorecent filament imaging and bead tracking) ensured, that a disturbing particle would be recognized during the measurement.

\subsubsection{Measuring Procedures}

In order to investigate the mechanical properties of vimentin filaments, several stretching procedures were used. For each individual measurement, that was taken into account for analysis, a fresh vimentin filament was captured. 

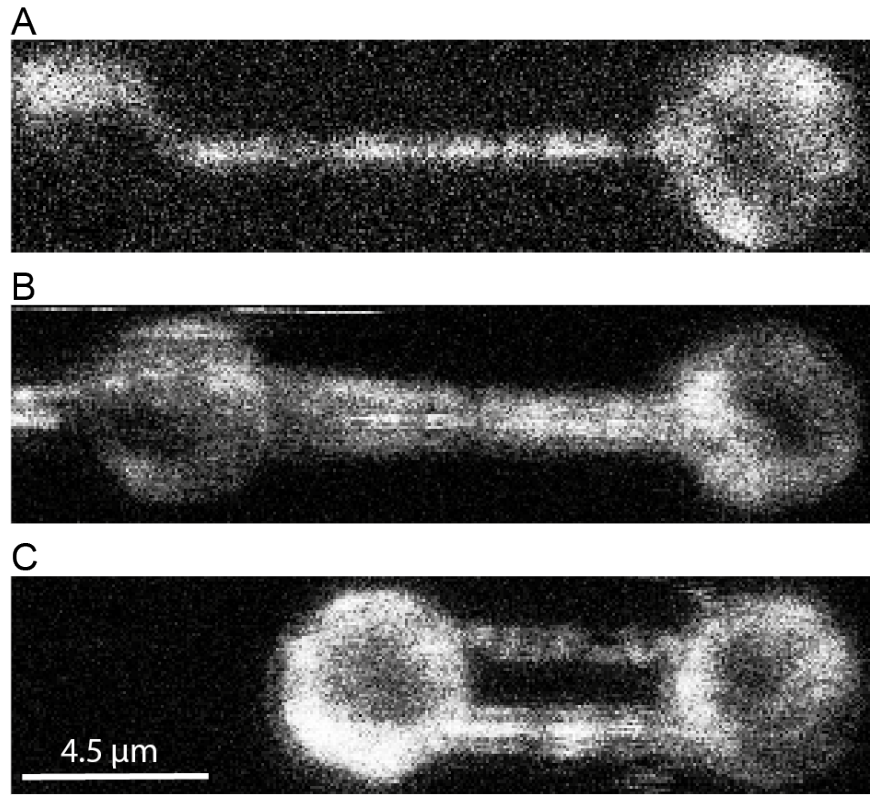

Figure 3.6: Fluorescence microscopy was used to ensure that only one filament has bound to both beads. Subfigures show confocal fluorescence images of (A) a single filament, (B) two or more filaments close to each other, (C) at least two clearly separated filaments.

Simple force-distance curves, as shown exemplarily in Fig. 3.7A were performed at different loading rates (or stretching velocities). A filament, captured between the two beads, was stretched until failure of the filament or the trap. Failure of the trap means that due to the increasing force the bead was pulled out of the trap while failure of the filament means that the filament was lost either due to rupture of the filament or due to breaking of the biotin-streptavidin bond. The last two cases are not easily distinguishable. For force-distance curves where not only the stretching but also the retraction curve was recorded, it was necessary to manually stop the bead-movement as close as possible to the maximum force of the trap but before a bead was pulled out of the trap. By clicking the reverse button immediately afterwards, retraction of the filament was started. However, due to the manually performed steps in this procedure, a little delay between the stretching and the retraction curve was technically unavoidable and can also be seen in the data sets. 
Force-distance cycles were performed either to increasing distances as shown in Fig. 3.7B, or to "constant" distances. Both procedures were executed at different loading rates. A plugin in the TWOM software was used to automate the cycles. In this plugin a start- and an end-position for the cycles was set. For cycles to increasing distances a number of equal steps to get from the start-to the end-position was defined. For cycles to constant distances the number of cycles was selected, additionally to the startand end-position.

The position, the second bead was moved to, was not optically measured as the real distance between the two beads, but the trap was moved to a specific position. Due to the filament stretching, a force was acting on the bead, pulling it out of the center of the trap. Therefore, the position of the bead and the center of the trap did not match while a force, especially a high force, was applied. As the filaments' properties changed due to repeated stretching, the distance between the bead and the center of the trap changed with each stretching cycle. Consequently, due to the softening of the filament, the actual distance between the two beads increased with each cycle, even though, for constant-distance cycles, the trap moved to the same position every time. For data acquisition this means that cycles to a constant distance were actually not possible, especially at higher forces.

An example data set of a force clamp measurement is shown in Fig. 3.7C. For those measurements a force clamp plugin in the TWOM software was used, which calculated the difference between the actual force and the nominal value and subsequently adjusted the force by moving the second bead further away from the first bead. While using this plugin, it was not possible to define the loading rate for the stretching, but the software tried to reach the set force as fast as possible. Force Clamp measurements were performed at several forces between 50 and $700 \mathrm{pN}$ and for 15 minutes to 8 hours.

Relaxing or creep experiments were performed starting from specified forces. Filaments were stretched until the set force was reached, using the force clamp plugin which was subsequently disabled and the filaments allowed to relax under force. Due to the physics of the optical trap, also in this experiment a really constant distance during the relaxing process was not possible as the beads, especially at higher forces, were displaced from the center. While the filament relaxed, the beads more and more returned to the center of the traps and thereby increased the distance between them. 
For the filaments this means that, even though there was no active force applied on the beads, the restoring force or spring constant of the trap (which was about $50 \mathrm{pN} / \mathrm{nm}$ ) was still acting.

Simple force-distance curves, force-distance cycles and force clamps were repeated with glutaraldehyde fixed vimentin filaments. For those experiments, the filaments were captured between two beads and moved from channel 3 (buffer channel) to channel 4 (buffer, containing $0.5 \%$ glutaraldehyde) by moving the flow cell with respect to the optical traps. After an incubation time of 5 minutes, the filaments were returned to channel 3 for the actual measurement. Moving the filament in direction of the filaments long axis was very robust, while moving perpendicular to the filaments long axis (moving into channel 4) had to be carried out very carefully.

\subsubsection{Cleaning Protocol for the Flow Cell}

Between measurements of the same series, the flow cell was cleaned by flushing with $1 \mathrm{~mL} \mathrm{NaOH}$ per syringe. Afterwards, assembly buffer was flushed until the $\mathrm{pH}$ at the exit of the microfluidic system was 7.5 again. Before a set of measurements was started, the flow cell was cleaned following a protocol regularly used in the group of E. Petermann and G. Wuite. This protocol uses a bleach solution of less than $5 \% \mathrm{NaOCl}$, whose hypochloric ion has strongly oxidizing properties and, due to the fact that the corresponding hypochloric acid is weak, $\mathrm{NaOCl}$ is almost as basic as $\mathrm{NaOH}$. The hypochloric acid, which is formed by dilution, is metastable and decomposes into chlorine gas, oxygen and water, thus enhancing the cleaning properties compared to $\mathrm{NaOH}$. During this cleaning protocol remaining biological material or dyes will be degraded and washed out of the flow cell. The procedure requires the following steps (water always means MilliQ water and all channels/syringes are treated the same way):

1. Syringes are fully filled with water, everything except $0.5 \mathrm{~mL}$ per syringe is removed by pipetting and all channels are briefly flushed with about $200 \mu \mathrm{l}$ of the remaining water.

2. Remaining water is removed and all syringes are filled with about $700 \mu \mathrm{l}$ bleach solution. To fill the whole flow cell with bleach solution channels are flushed 
rapidly first, thereafter a week flow is applied for 20 to $30 \mathrm{~min}$ until the syringes are nearly empty.

3. $1.5 \mathrm{~mL}$ water are added per channel and all channels are briefly flushed with about $200 \mu \mathrm{l}$.

4. Residual water is removed and, in case of very persistent dirt, all syringes are filled with $1 \mathrm{~mL}$ of $1 \mathrm{M} \mathrm{HCl}$ and channels are flushed briefly (about $200 \mu \mathrm{l}$ per channel).

5. All remaining solutions are removed and the syringes are fully filled with water. After briefly flushing with about $200 \mu$, residual water in the syringes is replaced by $1.5 \mathrm{~mL}$ fresh water.

6. Sodium thiosulfate $\left(\mathrm{Na}_{2} \mathrm{~S}_{2} \mathrm{O}_{3}\right)$ is added to each syringe to a final concentration of about $10 \mathrm{mM}$. All channels are flushed for about $10 \mathrm{~min}$.

7. Residual solutions are removed from the syringes and the system is thoroughly flushed with water.

After this cleaning protocol was completed, buffer was flushed in all channels first. Lastly, the sample, beads and, if needed, further compounds were added to the system.

\subsection{Data Processing}

Each optical trap data set consists of a table, containing the measurement values as floating point numbers, and a meta-data file, containing information about the settings of the setup and the user settings (e.g. camera settings like ROI and acquisition time/rate, bead size, experiment description), about the force calibration (e.g. corner frequency, trap stiffness) and about the file itself (e.g. molecule number, date and time).

As the trap software was LabView based, these files are saved in a LabView data format and have to be converted for further processing. The TWOM data viewer, developed in the Wuite and Peterman lab, can be used to have a quick look on the data and export the table of measurement data to either ASCII-format or as an Origin-file (OriginLab, Northampton, MA, USA). 

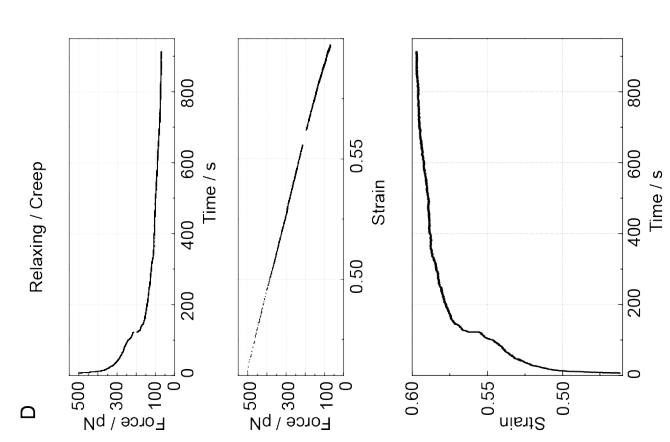

ชิ

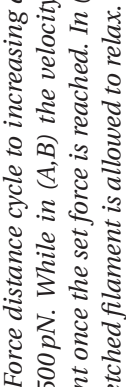
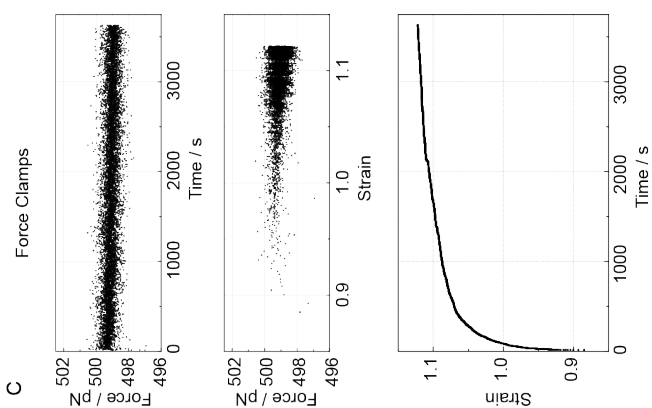

๑ิ

$\approx$ b

ఖั

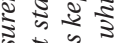

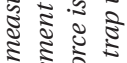

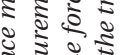

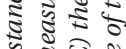

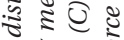

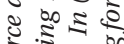

จ.

एँ

ह 5.

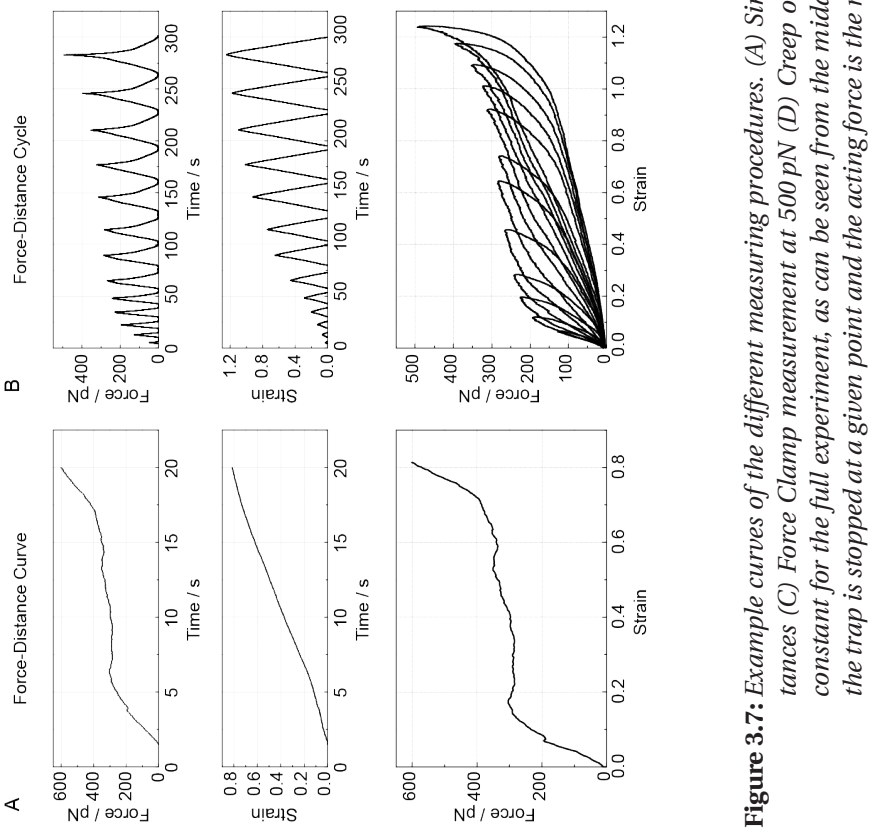


For further data processing MATLAB (The MathWorks, Inc., Natick, MA, USA, versions 2014b to 2016a) and Origin 8.5G (OriginLab, Northampton, MA, USA) was used. Additional data analysis, simulations and mathematical modelling, performed by Hannes Witt, were mainly conducted using the open source programs Python (Python Software Foundation, python.org) and Jupyter (jupyter.org).

\subsubsection{Processing for further analysis}

Depending on the experiment type, minimum requirements of filament stability were defined and will be further explained in the description of the procedure-specific processing.

Some steps were performed due to software settings and software failure. The TWOM software uses commas as decimal separator, which had to be replaced by points for further data processing. And, as described above, the end-to-end distance of the filament is monitored by bright-field imaging of the trapped beads (bead center to bead center). This bead-tracking did not always perform accurately over a complete measurement. Data sets, where the bead tracking failed over several consecutive data points, with the effect that it was not possible to follow the overall development of the force-distance curve, were rejected for any further analysis. Depending on the experiment and on the loading rate, this can mean that already two consecutive missing data points were two much (high loading rate cycles) or that 50 consecutive missing data points were no problem (long-lasting force clamps).

The following steps were performed for every data set, that was taken into account, prior to any experiment-specific analysis. Except the first one, all steps were carried out using MATLAB.

1. Data were exported to ASCII-format using the TWOM data viewer.

2. From the full data sheets, the relevant columns (time, bead-distance and force) were saved to new files, which were used for all further steps.

3. Commas were replaced by points.

4. (If necessary) data rows, where the bead tracking had failed, were deleted. 
5. To compensate for the bead tracking two bead radii were subtracted from the distance measurement.

6. We defined the filament length as the distance value corresponding to the last force value below $5 \mathrm{pN}$ during the initial stretching. This was the point at which, for most of the filaments, force and distance started to increase steadily. Below $5 \mathrm{pN}$ a lot of fluctuations in the force curve occurred.

7. The strain $\epsilon$ was calculated as $\epsilon=\frac{\Delta L}{L}$, where $L$ is the length of the filament and $\Delta L$ the difference between the length of the stretched filament and $L$.

8. Data points recorded before the initial filament length was reached and data points recorded after the trap or the filament failed were neglected for analysis.

\subsubsection{Processing Force-Distance Curves}

Only filaments where a force of at least $450 \mathrm{pN}$ was reached were taken into account for analysis. This ensured that for all loading rates the three different domains of the force-distance curve were reached.

The loading rate was set as a parameter in the operating software of the optical traps. However, the trap speed did vary from measurement to measurement. By linear fitting the measured distance over time, the actual loading rate was calculated for every single measurement. This fit was performed for the distance values corresponding to the force values between 50 and $450 \mathrm{pN}$ to account for some fluctuations in the loading rate at the beginning of each measurement and due to the fact, that the shortest data sets ended at a force of $450 \mathrm{pN}$.

The average of all data sets of one loading rate was calculated using the MATLAB toolbox "adsmoothdiff" written by Carlos J. Dias. This toolbox is based on the theory described in Ref. $[9,10]$. As a first step it was necessary to define a vector with x-values, which was used for all data sets of one loading rate. Using the data set, that reached the highest strain, the new $\mathrm{x}$-vector was defined as a vector that reached from 0 to the maximum strain $\epsilon_{\max }$ in 100 equal steps. Applying "adsmoothdiff" y-values corresponding to the new defined $x$-values were calculated for all data sets by smoothing and interpolation. Only for the data set which reached $\epsilon_{\max }$ the y-vector contained val- 
ues corresponding to all x-values. For the other data sets missing $\mathrm{y}$-values were filled with "NaN". The new calculated y-values were used to calculate the mean stretching curve per loading rate. All data sets were fitted individually by Hannes Witt applying the "two-state" model described in Ref. [1] and Chapter 4.

\subsubsection{Processing of Force Clamp and Creep Data}

As it was experimentally not possible to immediately apply a nominal force, data sets of force clamp and creep experiments consisted of two parts, the inital phase where the force increased from zero to the desired value and the actual clamp or creep experiment. Therefore, only data points which were recorded while the desired force value was applied on the filament, were used for analysis of force clamps. For analysis of creep experiments only data points recorded after the applied active force was switched off were taken into account.

Step sizes in the data sets were analyzed by plotting the filament length as a histogram. The procedure is explained in Chapter 5 and was performed by Hannes Witt.

\subsubsection{Correction of Force Offset}

In general, the force calibration and therefore the measurements were very stable and very easily comparable. Probably due to dirt on the objective, one set of measurements at the C-Trap in Amsterdam had very different values for the force calibration (spring constant) than all other measurements. To still gain comparable data, the data with the calibration outliner was corrected by dividing the force values by the spring constant of the calibration that was performed for this measurements. The new values were multiplied by the mean spring constant of the calibrations of the same experiments, performed during the other weeks of measurement. Data sets, corrected by this procedure, fitted very well to the other experimental data. No other correction procedures were performed for any of the optical trap data sets. 
Table 3.1: List of Chemicals and Enzymes

Chemical/Protein

AMP

ATTO $647 \mathrm{~N}$-maleimide

Bio-Gel P medium

biotin-maleimide

BSA

CM sepharose

L-cysteine

DEAE sepharose

DMSO

DNAse1

$\mathrm{C}_{2} \mathrm{H}_{5} \mathrm{OH}$

EDC

EDTA

EGTA

glutaraldehyde

glycerol
Full Name, Company

ampicillin sodium salt, Sigma-Aldrich, Munich, Germany

ATTO-TEC GmbH, Siegen, Germany

BioRad, Hercules, CA, USA

Jena Bioscience GmbH, Jena, Germany

bovine serum albumin, Sigma-Aldrich, Munich,

Germany, A7906-10G, Lot: SLBB476V

column material, GE Healthcare, Munich, Ger-

many

Carl-Roth GmbH, Karlsruhe, Germany

column material, GE Healthcare, Munich, Ger-

many

$\geq 99,8 \%$, p.A., dimethyl sulfoxide, Carl-Roth

GmbH, Karlsruhe, Germany

Sigma-Aldrich, Munich, Germany

ethanol, Carl-Roth GmbH, Karlsruhe, Germany

1-Ethyl-3-(3-dimethylaminopropyl)carboxi-

imide), Sigma-Aldrich, Munich, Germany,

E1769-1G

ethylenediaminetetraacetic acid, Carl-Roth

GmbH, Karlsruhe, Germany

ethylene glycol bis( $\beta$-aminoethyl)-ether

N,N,N',N' tetraacetic acid, Carl-Roth GmbH,

Karlsruhe, Germany

Polysciences Europe GmbH, Hirschberg an der

Bergstrasse, Germany

Carl-Roth GmbH, Karlsruhe, Germany 


\begin{tabular}{|c|c|}
\hline Chemical/Protein & Full Name, Company \\
\hline $\mathrm{HCl}$ & $\begin{array}{l}\text { hydrochloric acid, Carl-Roth GmbH, Karlsruhe, } \\
\text { Germany }\end{array}$ \\
\hline DOC & $\begin{array}{l}3 \alpha \text {-12 } \alpha \text {,dihydroxy- } 5 \beta \text {-cholanic acid sodium } \\
\text { salt, Sigma-Aldrich, Munich, Germany }\end{array}$ \\
\hline InstantBlue $^{T M}$ & $\begin{array}{l}\text { BIOZOL Diagnostica Vertrieb GmbH, \#EXP- } \\
\text { ISBO1L, Eiching, Germany }\end{array}$ \\
\hline LB agar plate & $\begin{array}{l}\text { lysogeny broth agar plate with ampicillin, Sig- } \\
\text { ma-Aldrich, Munich, Germany, L5667 }\end{array}$ \\
\hline lysozyme & Roche Diagnostics, Mannheim, Germany \\
\hline $\mathrm{MgCl}_{2}$ & $\begin{array}{l}\text { magnesium dichloride, Sigma-Aldrich, Munich, } \\
\text { Germany }\end{array}$ \\
\hline Maleimide-PEG-NH & $\begin{array}{l}\text { MW 5000, PG2-AMM2-5k, Nanocs, New York, } \\
\text { NY, USA }\end{array}$ \\
\hline MES buffer & $\begin{array}{l}\text { 2-(N-Morpholino)ethanesulfonic acid sodium } \\
\text { salt Sigma-Aldrich, Munich, Germany }\end{array}$ \\
\hline MAC & $\begin{array}{l}\text { methylamine hydrochloride, Sigma-Aldrich, } \\
\text { Munich, Germany }\end{array}$ \\
\hline NP40 & $\begin{array}{l}\text { nonyl phenoxypolyethoxylethanol, Roche Diag- } \\
\text { nostics, Mannheim, Germany }\end{array}$ \\
\hline Pefabloc SC & $\begin{array}{l}\text { serine protease inhibitor, Carl-Roth } \mathrm{GmbH} \text {, } \\
\text { Karlsruhe, Germany }\end{array}$ \\
\hline PMSF & $\begin{array}{l}\text { phenylmethylsulfonyl fluoride, Serva, Heidel- } \\
\text { berg, Germany }\end{array}$ \\
\hline protein marker & VWR, A5418.0250, Darmstadt, Germany \\
\hline PBS & $\begin{array}{l}\text { phosphate buffer saline, Invitrogen AG, Carls- } \\
\text { bad, CA, USA, LOT: 73604799A }\end{array}$ \\
\hline $\mathrm{KCl}$ & $\begin{array}{l}\text { potassium chloride, Carl-Roth GmbH, Karls- } \\
\text { ruhe, Germany }\end{array}$ \\
\hline RNAse & Roche Diagnostics, Mannheim, Germany \\
\hline
\end{tabular}


Chemical/Protein

saccharose

sample buffer for SDS-gel

$\mathrm{NaCl}$

$\mathrm{NaH}_{2} \mathrm{PO}_{4}$

$\mathrm{NH}_{2}$-PEG-OH

SDS

SDS-gel

$\mathrm{Na}_{2} \mathrm{HPO}_{4}$

$\mathrm{NaHSO}_{3}$

TB

DTT

tricine

TRIS-HCl

Triton X100

$\mathrm{CH}_{4} \mathrm{~N}_{2} \mathrm{O}$
Full Name, Company

D-sucrose, $\geq 99,5 \%$, p.A., Carl-Roth $\mathrm{GmbH}$,

Karlsruhe, Germany

VWR/CBS, \#FB31010, Darmstadt, Germany

sodium chloride, Carl-Roth GmbH, Karlsruhe,

Germany

sodium dihydrogen phosphate dihydrate, Carl-

Roth GmbH, Karlsruhe, Germany

Iris-Biotech, Marktredwitz, Germany

sodium dodecyl sulphate, Carl-Roth $\mathrm{GmbH}$,

Karlsruhe, Germany

VWR/CBS, \#FK00812-10, Darmstadt, Germany

disodium hydrogen phosphate dihydrate, Carl-

Roth GmbH, Karlsruhe, Germany

sodium hydrogensulfit, Carl-Roth GmbH, Karls-

ruhe, Germany

terrific broth, Sigma-Aldrich, Munich, Germany, \#T0918

1,4-di-thiothreitol, Carl-Roth GmbH, Karlsruhe,

Germany

$\mathrm{N}$-[tris(hydroxymethyl)methyl]glycine, Carl-

Roth GmbH, Karlsruhe, Germany

Tris(hydroxymethyl) aminomethane, Carl-Roth

GmbH, Karlsruhe, Germany

4-(1,1,3,3-tetramethylbutyl)phenyl-

polyethylene glycol, Carl-Roth GmbH, Karls-

ruhe, Germany

urea, Carl-Roth GmbH, Karlsruhe, Germany 
Chapter 3. Materials and Methods

\section{References}

1. Block, J. et al. Nonlinear Loading-Rate-Dependent Force Response of Individual Vimentin Intermediate Filaments to Applied Strain. Phys. Rev. Lett. 118, 048101 (2017).

2. Block, J. et al. Viscoelastic properties of vimentin originate from nonequilibrium conformational changes. Sci. Adv. 4, eaat1161 (2018).

3. Herrmann, H. \& Aebi, U. Intermediate Filaments: Molecular Structure, Assembly Mechanism, and Integration into Functionally Distinct Intracellular Scaffolds. Annu. Rev. Biochem. 73, 749-789 (2004).

4. Winheim, S. et al. Deconstructing the Late Phase of Vimentin Assembly by Total Internal Reflection Fluorescence Microscopy (TIRFM). PLOS One 6, e19202 (2011).

5. Nöding, B., Herrmann, H. \& Köster, S. Direct Observation of Subunit Exchange along Mature Vimentin Intermediate Filaments. Biophys J 107, 2923-2931 (2014).

6. Janissen, R. et al. Invincible DNA Tethers: Covalent DNA Anchoring for Enhanced Temporal and Force Stability in Magnetic Tweezers Experiments. Nucleic Acids Res. 42, e137 (2014).

7. Gross, P., Farge, G., Peterman, Erwin J. G. \& Wuite, Gijs J. L. Combining Opticalt Tweezers, Single-Molecule Fluorescence Microscopy, and Microfluidics for Studies of DNA-Protein Interactions. Method. Enzymol. 475, 427-453 (2010).

8. Candelli, A., Wuite, Gijs J L \& Peterman, Erwin J G. Combining Optical Trapping, Fluorescence Microscopy and Micro-Fluidics for Single Molecule Studies of DNAProtein Interactions. Phys. Chem. Chem. Phys. 13, 7263-7272 (2011).

9. Savitzky, A. \& Golay, M. J. E. Smoothing and Differentiation of Data by Simplified Least Squares Procedures. Anal. Chem. 36, 1627-1639 (1964).

10. Barak, P. Smoothing and Differentiation by an Adaptive-Degree Polynomial Filter. Anal. Chem. 67, 2758-2762 (1995). 


\section{Chapter 4}

\section{Nonlinear Loading-Rate-}

\section{Dependent Force Response of Individual Vimentin}

\section{Intermediate Filaments to}

\section{Applied Strain}

This chapter was published as "Nonlinear Loading-Rate-Dependent Force Response of Individual Vimentin Intermediate Filaments to Applied Strain" (J. Block et al., Physical Review Letters 118, 048101, ISSN : 0031-9007 (4 2017), DOI: 10.1103/PhysRevLett.118.048101), and reproduced in this dissertation with the permission of the American Physical Society under the terms of the Creative Commons Attribution 3.0 License.

Johanna Block ${ }^{1}$, Hannes Witt ${ }^{2}$, Andrea Candelli ${ }^{3,4}$, Erwin J. G. Peterman ${ }^{3}$, Gijs J. L. Wuite $^{3}$, Andreas Janshoff ${ }^{2}$, Sarah Köster ${ }^{1}$ 
Chapter 4. Nonlinear Loading-Rate- Dependent Force Response of Individual Vimentin Intermediate Filaments to Applied Strain

${ }^{1}$ Institute for X-Ray Physics, University of Goettingen, 37077 Göttingen, Germany.

${ }^{2}$ Institute of Physical Chemistry, University of Goettingen, 37077 Göttingen, Germany.

3 Department of Physics and Astronomy and LaserLab, Vrije Universiteit Amsterdam, 1081 HV Amsterdam, Netherlands.

${ }^{4}$ LUMICKS B.V., 1081 HV Amsterdam, Netherlands.

Contribution to the article: Vimentin has been produced and isolated by Susanne Bauch. I have prepared all buffers and the vimentin filaments for all experiments. I have also performed and optimized the experiments and analyzed the data of the optical tweezers experiments. Hannes Witt performed the AFM experiments and analyzed the AFM data. I produced all figures for the manuscript. The article was written and then iteratively optimized by Hannes Witt, Sarah Köster, Andreas Janshoff and myself. 


\subsection{Abstract}

The mechanical properties of eukaryotic cells are to a great extent determined by the cytoskeleton, a composite network of different filamentous proteins. Among these, intermediate filaments (IFs) are exceptional in their molecular architecture and mechanical properties. Here we directly record stress-strain curves of individual vimentin IFs using optical traps and atomic force microscopy. We find a strong loading rate dependence of the mechanical response, supporting the hypothesis that IFs could serve to protect eukaryotic cells from fast, large deformations. Our experimental results show different unfolding regimes, which we can quantitatively reproduce by an elastically coupled system of multiple two-state elements.

\subsection{Introduction}

The eukaryotic cytoskeleton is a crucial determinant of many cellular properties, including cell mechanics. Three types of filamentous proteins, microfilaments (MFs), microtubules (MTs) and intermediate filaments (IFs) interact with numerous crosslinking proteins and molecular motors, and the emerging composite network spans the whole cell [1]. Whereas MFs and MTs are conserved across tissues and organisms, IFs are expressed in a cell-type specific manner and 70 different IF coding genes have been found in humans [2]. Interestingly, IFs have recently been found to play a direct role in cell mechanics $[3,4]$. All cytoskeletal IFs share a tripartite monomer structure consisting of a $\alpha$-helical "rod" domain and intrinsically disordered "head" and "tail" regions (see Fig. 4.1a, top). The molecular assembly of IFs follows a strictly hierarchical path, including lateral assembly into dimers, tetramers and unit length filaments (ULFs), followed by longitudinal annealing leading to $10 \mathrm{~nm}$ diameter filaments that can be many $\mu \mathrm{m}$ long, as shown schematically in Fig. 4.1a, bottom.

IFs show interesting mechanical properties [2, 8-10] such as high flexibility, with a persistence length of few $\mu \mathrm{m}[11,12]$ and extreme extensibility [13, 14]. Both properties originate from the open molecular architecture of the filaments. The extensibility of different IFs has previously been measured by lateral stretching of adsorbed IFs using atomic force microscopy (AFM) [13-15] and force-strain curves for vimentin dimers 


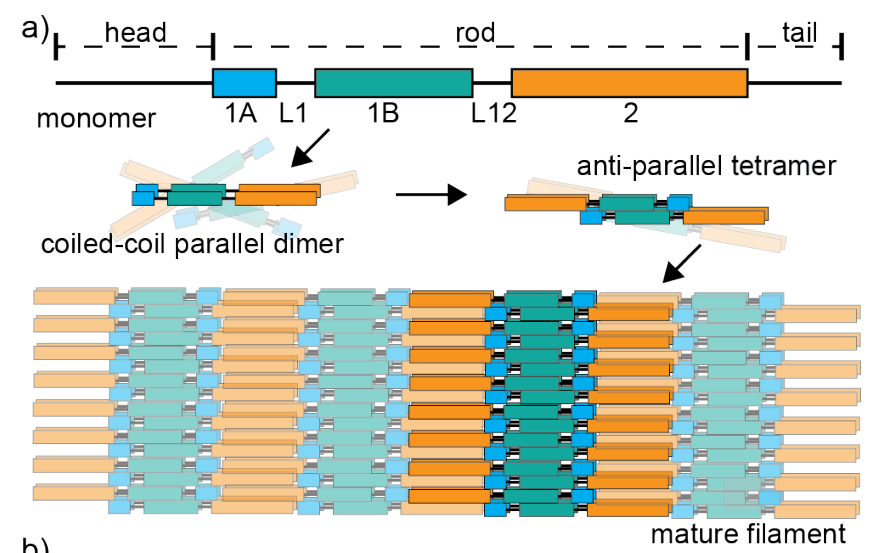

b)

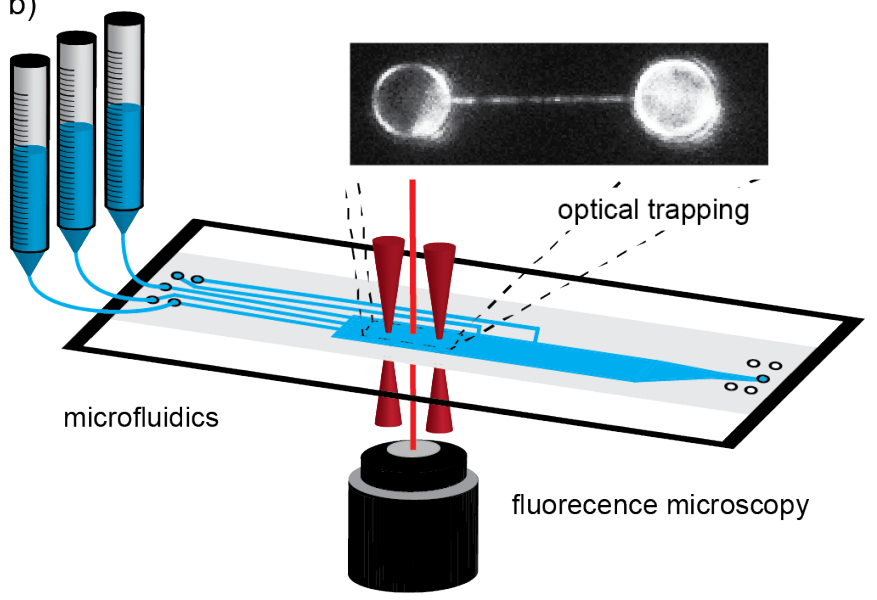

Figure 4.1: a) Schematic representation of vimentin filament formation. Vimentin monomers contain a head, a tail and a central rod domain. The rod domain is composed of three $\alpha$-helical coils (1A: 36 amino acids (aa), 1B: $92 \mathrm{aa}$ and 2: $139 \mathrm{aa}$ ) and two linkers (L1 and L12) [5-7]. Two monomers form a parallel coiled-coil dimer and two dimers form a half-staggered anti-parallel tetramer. Via unitlength-filaments (ULFs, composed of typically eight tetramers, not shown) mature filaments are formed by longitudinal annealing. b) Overview of the experimental setup used for OT experiments, combining optical traps and confocal imaging with a microfluidic device. The inset shows a fluorescently labeled vimentin filament trapped between two beads.

and tetramers have been modeled by molecular dynamics simulations [16]. The AFM experiments have revealed that IFs can be extended up to 3.6 times the original length before they rupture. However, it is in the nature of the measurement that the filaments 
may be imaged before and after stretching, but not in situ during the process. Since the filaments are stretched laterally and interact with the substrate during the extension a precise and quantitative assessment of the elastic properties could not be achieved.

Here, we directly probe the loading rate dependent mechanical properties of individual vimentin IFs under physiological buffer conditions using optical traps (OT) and AFM to stretch the filaments along their long axis up to a strain of 3.5. A strong loading rate dependency of the mechanical response is found and explained by an elastically coupled system of multiple two-state elements.

\subsection{Materials and Methods}

Human vimentin C328A with an additional cysteine at the C-terminus was recombinantly expressed in E. coli and purified from inclusion bodies [17]. Labeling with ATTO647N-maleimide (AttoTech GmbH, Siegen, Germany) or biotin-maleimide (Jena Bioscience GmbH, Jena, Germany) was performed according to Ref. [18] with minor changes for the biotin labeling. The biotin-labeled vimentin and any remaining free biotin were separated by 2 x elution over PD25 Miditrap columns (GE Healthcare Freiburg, Germany). In the first run, the column was equilibrated with labeling buffer and eluted with strorage buffer (buffers according to [18]). In the second run the column was equilibrated and eluted with storage buffer. The protein concentration was monitored by measuring the absorption at $280 \mathrm{~nm}$ (Nanodrop ND-1000, ThermoScientific Technologies, Inc.,Wilmington, USA). Labeled and unlabeled vimentin was stored at $-80^{\circ} \mathrm{C}[12,18]$. Prior to assembly into filaments, vimentin protein was dialyzed stepwise into $2 \mathrm{mM}$ sodium phosphate buffer, pH 7.5 [12]. Assembly was performed at a vimentin concentration of $0.2 \mathrm{~g} / \mathrm{L}$ by dialysis into $2 \mathrm{mM}$ sodium phosphate buffer, $\mathrm{pH} 7.5$, containing $100 \mathrm{mM} \mathrm{KCl}$ (assembly buffer), at $37^{\circ} \mathrm{C}$ over night [19]. About $2 \%$ of the monomers were ATTO647N labeled and about $15 \%$ were biotin labeled. Before OT and AFM measurements, vimentin filaments were diluted 1:1000 and 1:10 with assembly buffer, respectively.

OT measurements were performed with a setup combining optical traps, a confocal fluorescence microscope and a microfluidic system (LUMICKS, Amsterdam, Netherlands) [20, 21] (Fig. 4.1b). Streptavidin-coated polystyrene beads (4.5 $\mu \mathrm{m}$, Spherotech, 
Chapter 4. Nonlinear Loading-Rate- Dependent Force Response of Individual

Vimentin Intermediate Filaments to Applied Strain

Inc., Lake Forest, IL, USA), filaments and buffer were flushed separately into the microfluidic channel by air pressure. Due to laminar flow, the three solutions did not mix in the channel part where the measurements were executed. Every measurement was started by capturing two beads with the OTs. By moving the microfluidic device with respect to the OTs, filaments were attached to the beads and moved into the buffer channel. Subsequently stopping the flow ensured that only one filament was attached between the to beads, as controlled by imaging (see movie in the Supplemental Material [22]). Filaments were stretched to a maximum force of about $800 \mathrm{pN}$ with different loading rates, varying from $0.05 \mu \mathrm{m} / \mathrm{s}$ to $4.5 \mu \mathrm{m} / \mathrm{s}$.

AFM experiments were performed using a commercial instrument (MFP-3D Bio, Asylum Research, Santa Barbara, CA, USA). Vimentin filaments were attached to a freshly cleaved mica surface by physisorption. Cantilevers (MLCT, Bruker, Billerica, MA, USA) were calibrated using the thermal noise method; spring constants were found to be 50 to $60 \mathrm{pN} / \mathrm{nm}$. Stretching was performed by catching the freely fluctuating end of only partly attached vimentin filaments with the cantilever via physisorption and repeatedly displacing the cantilever from the mica surface with a loading rate of $5 \mu \mathrm{m} / \mathrm{s}$. A final force of about $8 \mathrm{nN}$ was reached without breaking the filaments.

\subsection{Results}

The raw data (force-distance curves) are processed by self-written MatLab code to obtain force-strain curves for every filament. The strain is defined as $\epsilon=\Delta L / L$, where $\Delta L$ is the difference between the length of the extended filament and the length of the filament $L$. The unstretched length $L$ is measured at the largest measured force before reaching 5 pN. The individual experimental data sets are shown in Fig. 4.2 (thin lines), where each color represents one loading rate, altogether covering two orders of magnitude. The averages of the measured curves are calculated using a MatLab Toolbox written by Carlos J. Dias applying the theory described in Refs. [23, 24] and are represented by the thick lines.

In the individual OT data sets, three different regimes can be discerned. Comparison of our data to literature suggests that the steep linear increase at low strain represents the elastic stretching of the $\alpha$-helical domains [16, 25, 26], whereas during the second, 


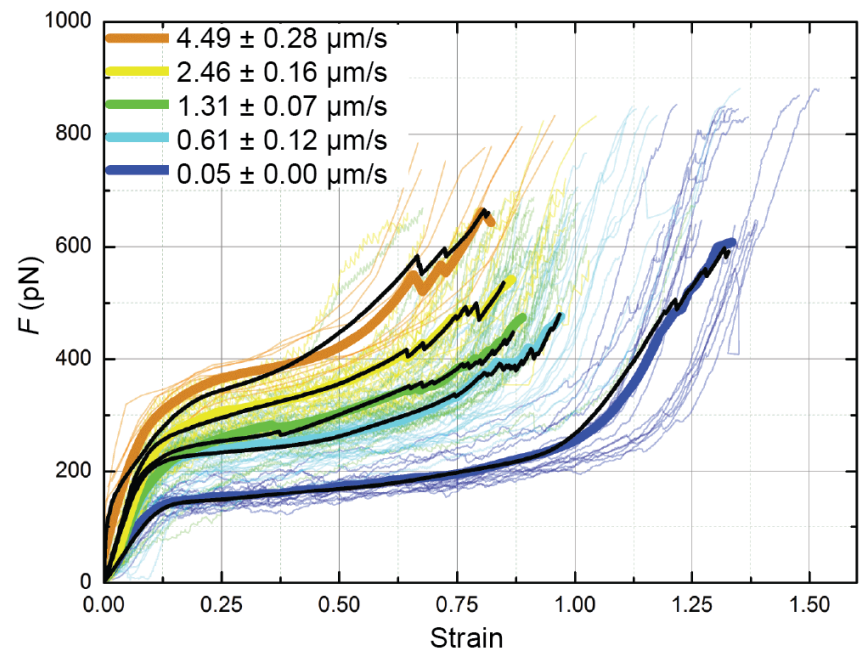

Figure 4.2: Force-strain curves recorded with the OT at different loading rates (color code, see legend) of individual vimentin filaments (thin lines) and corresponding average (thick line). The black lines show the averaged fits for each stretching loading rate.

plateau-like regime starting at a strain of about 0.1 the $\alpha$-helices are uncoiled and $\beta$ sheets are formed instead [16, 25, 27]. In the third regime a clear stiffening with respect to the plateau region can be observed which, according to simulations [16], might be due to increased pulling on the $\beta$-sheets. Previously, it has been shown that cells are indeed able to resist strains in the same order of magnitude as reached here [8].

Furthermore, we observe a clear loading rate dependence of the force-strain curves. With increasing loading rate the force value of the plateau region increases and strain range of the plateau regime decreases. Whereas the strain at which the plateau is reached does not change much between the loading rates, the strain at which the stiffening starts decreases with increasing loading rate.

To describe the force-extension-curves of vimentin filaments in the strain range accessible by OT we adopt an elastically coupled two-level model introduced by Rief et al. [28]. In brief, the filament is modeled as a system of entropic springs coupled to an elastic element (Fig. 4.3a). Each monomer can assume different states with distinct lengths and energies, in case of vimentin a shorter $\alpha$-helical ground state of length $l_{\alpha}$ and an extended $\beta$-sheet of length $l_{\beta}$. It is known that the vimentin monomer consists 


. Vimentin Intermediate Filaments to Applied Strain

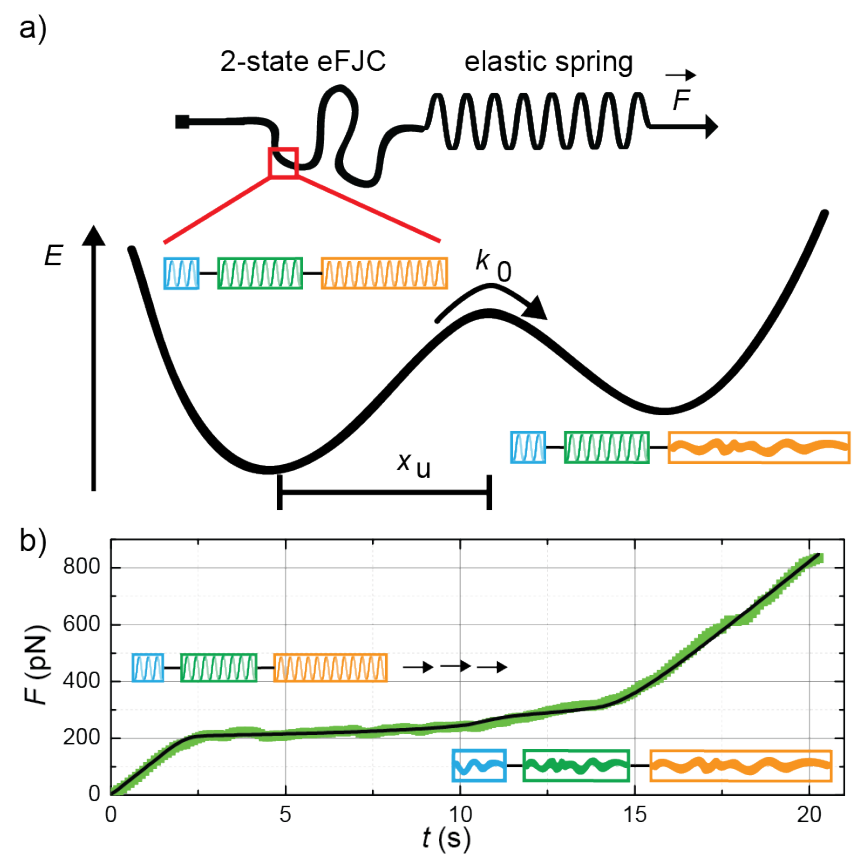

Figure 4.3: a) Sketch of the mechanical model consisting of a two-state eFJC and an elastic spring (top). A double-well potential is assumed to describe the unfolding reaction of the $\alpha$-helical subunits of a vimentin monomer (bottom). b) Eq. (4.1) fitted to a typical force-time curve of a single vimentin filament stretched at a loading rate of $0.6 \mu \mathrm{m} / \mathrm{s}$.

of three $\alpha$-helices, which are assumed to react independently to the applied force and are indicated by subscripts ( $i=1,2,3$ corresponding to the $1 \mathrm{~A}, 1 \mathrm{~B}$ and 2 domain) in the following. The $\alpha$-helices switch stochastically into a $\beta$-sheet conformation following a force-dependent Bell-Evans kinetic. This allows us to obtain the number $N_{\beta, i}$ of monomers $\frac{\mathrm{d} N_{\beta, i}}{\mathrm{~d} t}=\left(N_{0}-N_{\beta, i}\right) \cdot k_{0, i} \exp \left(\frac{F \cdot x_{\mathrm{u}, i}}{k_{\mathrm{B}} T}\right)$, where the $i$ th subunit is in the extended state. Here, the total number of monomers arranged in series is $N_{0}=N_{\alpha, i}+N_{\beta, i}$, the zero-force transition rate of the respective three subunits is $k_{0, i}$ and their potential width is $x_{\mathrm{u}, i}$. In the vimentin filament on average 32 monomers are arranged in parallel. We assume that parallel monomers are captured by this model by decreasing the potential width $x_{u}$ as described by Friddle et al. [29]. $x_{\mathrm{u}, i}$ is therefore an apparent value dependent on the number of parallel monomers. The contour length of the filament 
is $L_{\mathrm{c}}=N_{0} \cdot l_{0}+\sum_{i}\left(N_{\beta, i} \cdot l_{\beta, i}+N_{\alpha, i} \cdot l_{\alpha, i}\right)$, where $l_{0}$ is the combined length of linker regions. We use the equivalent Freely Jointed Chain (eFJC) model with the Kuhn length $L_{\mathrm{K}}$, which gives a good description in the force range observed experimentally [30], to model the entropic behavior of the filament under stress (see Supplemental Material [22] for a more detailed description of the model). In combination with an effective spring constant $k_{\text {eff }}$, which includes elastic behavior of the filament as well as the spring constant of the AFM cantilever or the OT, we obtain an expression for the timedependent force $F$ for a linear extension of the filament with constant loading rate $v$ :

$$
F(t)=k_{\text {eff }}\left(v \cdot t-L_{C}(F, t) \cdot\left(1-\frac{F_{K}}{F}\right)\right)
$$

Eq. (4.1) is solved analytically for $F$ using the numerical solution for the rate equation and fitted to the data. The model describes the experimental data remarkably well (see Fig. 4.3b for an example fit to an OT data curve). To reduce the number of fit parameters, $l_{\alpha, i}$ and $l_{\beta, i}$ are estimated from structural data [5-7]: $l_{\alpha, 1}=5.4 \mathrm{~nm}, l_{\alpha, 2}=13.8 \mathrm{~nm}$, $l_{\alpha, 3}=20.9 \mathrm{~nm}, l_{\beta, 1}=12.6 \mathrm{~nm}, l_{\beta, 2}=32.2 \mathrm{~nm}, l_{\beta, 3}=48.7 \mathrm{~nm}, l_{0}=29.7 \mathrm{~nm} . k_{0, i}$ is set to $k_{0, i}=3.3 \cdot 10^{-5} \mathrm{~s}^{-1}$, as found by Rief et al. for the unfolding of $\alpha$-helical spectrin repeats [31].

All experimental data curves are fitted individually with independent starting parameters. We find multiple local minima, i.e., sets of parameters able to describe all different experimental curves with little variance (see Supplemental Material [22]). The parameter set with the lowest variance is (mean $\pm \mathrm{SD}$ ) $\bar{x}_{\mathrm{u}, 1}=0.09 \pm 0.04 \mathrm{~nm}, \bar{x}_{\mathrm{u}, 2}=$ $0.12 \pm 0.04 \mathrm{~nm}$ and $\bar{x}_{\mathrm{u}, 3}=0.18 \pm 0.06 \mathrm{~nm}$. The effective spring constant of the filament was found to be $\bar{k}_{\text {eff }}=0.22 \pm 0.06 \mathrm{pN} / \mathrm{nm}$, corresponding to a Young's modulus $E=k_{\text {eff }} \frac{L}{\pi r^{2}} \approx 27 \pm 10 \mathrm{MPa}$, and a persistence length of $L_{P}=E \pi r^{4} /\left(4 k_{B} T\right) \approx 3.3 \mu \mathrm{m}$. Here, we include that the spring constants of the OTs and the AFM $(1.23 \mathrm{pN} / \mathrm{nm}$ and

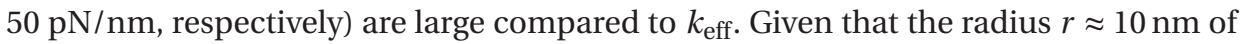
the filament enters the persistence length with a power of 4 , this result is in very good agreement with previous measurements of $L_{P}[11,12]$. The average of all individual fits for each loading rate in the same strain range as the curves of the averaged experimental data sets is shown as black lines in Fig. 4.2.

The OT setup provides superior control over the experiment due to the simulta- 
$\underline{74}$

Vimentin Intermediate Filaments to Applied Strain

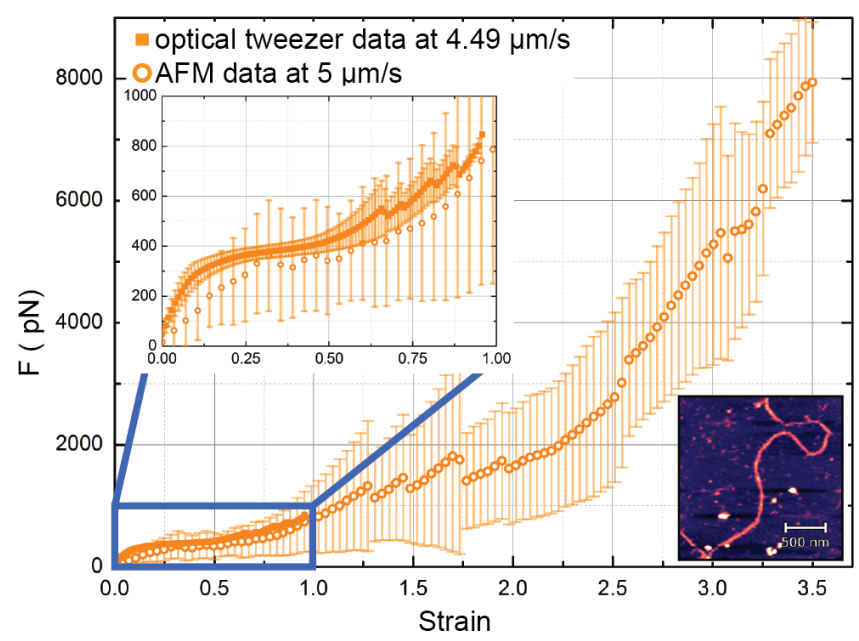

Figure 4.4: Comparison of AFM (open circles) and OT (solid symbols) experiments using approximately the same loading rate. The top left inset shows a zoom-in of the part where the data of OT and AFM measurements overlay. The bottom right inset shows an AFM image of vimentin filaments on a mica surface.

neous imaging modality and the microfluidic chip. However, the maximum forces reached are on the order of $800 \mathrm{pN}$ and Fig. 4.2 clearly shows that the maximum strain is not yet reached at these forces. Previous AFM experiments have shown that rupture of the filaments occurs at a strain of about 2.6 [13], whereas the maximum strain reached in our OT experiments is 1.5. Therefore, we compare force-strain curves obtained by OT with AFM data, as shown in Fig. 4.4. The open circles represent the average curve of all AFM measurements, including the standard deviations as error bars in direct comparison to the OT data (solid symbols) at a similar loading rate. In the AFM experiment the filament is stretched between the AFM tip and a mica surface, providing less control over the orientation of the filament compared to a filament trapped between two beads. Therefore, the variance of force between the individual filaments is higher than for OT data. However, the average curves of both experiments overlap remarkably well in the lower force regime confirming that we measure the response of individual filaments in the AFM experiments. Additionally, the AFM measurement allows to apply forces up to $8 \mathrm{nN}$ and therefore to reach a strain of up to 3.5 , albeit, 
still without breaking the filaments. For lower strains AFM force-strain curves replicate the three stress regimes found in the OT experiments. At larger strains, however, two additional regimes are observed: a second flat region with only little change in force at strains between 1.25 and 2, and a third linear regime at strains greater than 2 is found. A second force plateau has been predicted by molecular dynamics simulations, where it was assigned to sliding between dimers within a tetramer [16].

\subsection{Discussion}

In combination, OT and AFM measurements provide a precise and quantitative approach to the force-strain relationship for individual vimentin IFs. The data can be well described up to a strain of 1.2 by an elastically coupled system of multiple twostate elements supporting the hypothesis that subsequent stochastically independent unfolding of $\alpha$-helices into more extended $\beta$-sheet structures is responsible for the first force plateau and is thereby contributing to the remarkable extensibility of these filaments. When a critical force defined by $k_{0}$ and $x_{\mathrm{u}}$ is reached [32], the filament extends very rapidly leading to a force plateau as observed in the experiment. It is necessary to assume an independent reaction of the three $\alpha$-helical domains in the rod to capture the change in plateau length between force curves at different loading rates while keeping $l_{\beta, i}$ fixed at the values known from structural data. The preferred order in which the three subunits unfold is governed by the potential width $x_{\mathrm{u}, i}$ : The subunit with the largest potential width shows the strongest coupling to the applied force and has therefore the highest probability to unfold first. Interestingly, our fits indicate a high preference that coil 2 (corresponding to $i=3$ ) opens up first. The reason could be the socalled stutter region which is found precisely six heptads spaced from the C-terminal end of coil 2 in all IF types [33,34]. At this position the heptad repeat, characteristic of coiled-coils, is disrupted, either due to three missing or four extra amino acids [33, 35]. Notably this defect does not compromise the coiled-coil geometry but leads to an almost parallel run of the two $\alpha$-helices $[34,36]$ and therefore changes the angle between the direction of the applied force and the direction of the bonds that break during the stretching process [26]. Thus, the stutter region is less stable than an intact coiled-coil and might be the reason why coil 2 responds most sensitively to applied forces. From a 
Chapter 4. Nonlinear Loading-Rate- Dependent Force Response of Individual

Vimentin Intermediate Filaments to Applied Strain

biological point of view this is in agreement with the hypothesis that IFs act as a "safety belt" for cells [16], that is they are easily deformable and thus mechanically almost not detectable at slow deformation but stiff and therefore preserving the cell shape at fast deformation. This aspect is reflected in the shortened plateau regime (see Fig. 4.2) at higher loading rates. At slow deformation, the filaments can be extended to about 2 -fold their original length (strain of 1 ) at a comparatively low force. When pulled on faster, however, already at a strain of 0.5 , inceased forces are necessary for further extension. Our numerical model implies a simple mechanism for this observation based on the independent unfolding of the three individual coils forming the monomer. Forces as strong as applied by AFM here occur rarely in nature, therefore the second nonlinear regime may be interpreted as a controlled failure mechanism, allowing the filament to withstand extreme forces and large deformation.

Our data agree well with previous molecular dynamics simulations [16], not only with regard to the different observed regimes in the force-strain curve, but even concerning the numerical values for strains, even though Qin et al. only modeled stretching of dimers and tetramers. Within a fully assembled vimentin filament about 32 monomers, albeit with some variation to this number [37], are arranged in parallel. In our model we assume that both monomers in series and in parallel arrangement react independently. It is, however, expected that the monomers elongate in a coordinated way. Future experiments involving more sophisticated imaging in parallel to the mechanical stretching may help to approach these aspects.

In conclusion, our experimental results support the hypothesis that IFs provide strength to cells under large deformation and that unfolding enables them to absorb large amounts of energy [34, 38]. During a slow deformation vimentin acts as a very soft material, while a faster deformation shortens the plateau region and leads to a "stiffening" of the filament. More generally, the force dependent switching between $\alpha$-helices to $\beta$-sheets as well as breaking of lateral bonds between individual subunits can be understood in the light of molecular bond-breaking taking place on different levels of hierarchy in soft biological matter, as recently, shown by Kurniawan for the blood protein fibrin $[39,40]$. Thus, we believe that our study of individual cytoskeletal IFs will help to further quantify the underlying physical phenomena of soft matter mechanics. 


\section{Acknowledgements:}

We are grateful for fruitful discussions and technical support by J. Cabanas Danes, H.

Herrmann, F. C. MacKintosh and K. Kroy. The work was financially supported by the Deutsche Forschungsgemeinschaft (DFG) in the framework of SFB755 (project B7), SFB937 (project A17), and the GGNB (Grant No. GSC 226/2). The research leading to these results has received funding from Laserlab-Europe (Grant Agreement No. 654148, European Union's Horizon 2020 research and innovation programme). 
Chapter 4. Nonlinear Loading-Rate- Dependent Force Response of Individual

\subsection{Supplementary Information}

In this supplementary file we present a more detailed overview of the model we use to describe the data as well as the fit results.

\subsubsection{Theoretical model}

Following Rief et al. [28] we assume a force-dependent Bell-Evans kinetic for the stochastic switching between the $\alpha$-helical and the $\beta$-sheet state for each of the subunits $i=1,2,3$. Thus, the number $N_{\beta, i}$ of monomers with the $i$ th subunit in extended state is described by

$$
\frac{\mathrm{d} N_{\beta, i}}{\mathrm{~d} t}=\left(N_{0}-N_{\beta, i}\right) \cdot k_{0, i} \exp \left(\frac{F \cdot x_{\mathrm{u}, i}}{k_{\mathrm{B}} T}\right),
$$

with the total number of monomers $N_{0}=N_{\alpha, i}+N_{\beta, i} . k_{0, i}$ is the zero-force transition rate and $x_{\mathrm{u}, i}$ is the potential width. We calculate the contour length of the filament as

$$
L_{\mathrm{c}}=N_{0} \cdot l_{0}+\sum_{i}\left(N_{\beta, i} \cdot l_{\beta, i}+N_{\alpha, i} \cdot l_{\alpha, i}\right)
$$

where $l_{0}$ is the combined length of linker regions.

When stretched, the filament (length $x=x_{1}+x_{2}$ ) in the optical traps (OTs) or atomic force microscope (AFM) acts as a Hookian spring (length $x_{1}$, spring constant $k_{\text {eff }}$ ) in series with an entropic spring (length $x_{2}$ ), which we model as an equivalent Freely Jointed Chain (eFJC) with a Kuhn length $L_{\mathrm{K}}$. $L_{\mathrm{K}}$ sets the force scale $F_{\mathrm{K}}=\frac{k_{\mathrm{B}} T}{L_{\mathrm{K}}}$. Thus,

$$
x_{2}=L_{\mathrm{C}}(F, t)\left(\operatorname{coth}\left(\frac{F}{F_{\mathrm{K}}}\right)-\frac{F_{\mathrm{K}}}{F}\right) .
$$

In our experiments, $F_{\mathrm{K}}$ is on the order of $10^{-15} \mathrm{~N}$, therefore, we approximate

$$
x_{2} \approx L_{\mathrm{C}}(F, t)\left(1-\frac{F_{\mathrm{K}}}{F}\right)
$$

We write the force as

$$
F=k_{\text {eff }} x_{1}=k_{\text {eff }}\left(x-x_{2}\right)
$$


Applying a constant loading rate $v, x=v t$ and thus using Eqs. (4.5) and (4.3)

$$
F=k_{\text {eff }}\left(v t-L_{\mathrm{c}}\left(1-\frac{F_{\mathrm{K}}}{F}\right)\right)
$$

Eq. (4.7) can be solved analytically for $F$ and is used to fit the numerical solution of Eq. (4.2) with the boundary condition $N_{\alpha, i}=N_{0}$ for $t=0$ to the data. The total number of monomers $N_{0}$ is calculated from the length of the relaxed filament $\mathrm{L}$ and the length of the monomer in all- $\alpha$ configuration $N_{0}=\frac{L}{l_{0}+l_{\alpha, 1}+l_{\alpha, 2}+l_{\alpha, 3}}$. As described in the main text, $l_{0}, l_{\alpha, i}$ and $l_{\beta, i}(i=1,2,3)$ are obtained from structural data [5-7], the applied force $F$ and loading rate $v$ are obtained from the experimental data, and $k_{0, i}$ for an $\alpha$-helix to $\beta$-sheet transition is known from literature [31]. Thus, the free fit parameters are $k_{\text {eff }}$ and $x_{\mathrm{u}, i}(i=1,2,3)$, as shown below. The numerical solution of Eq. 4.7 is implemented in Python using the NumPy and SciPy packages [41].

\subsubsection{Comparison of Worm Like and Freely Jointed Chains}

As demonstrated by Toan and Thirumalai [30] an equivalent Freely Jointed Chain gives a good description for entropic chains in the force ranged observed experimentally. To demonstrate this, we compare it to a Worm Like Chain using the description of Odijk [42]. This changes Eq. 4.5 to

$$
x_{2}=L_{\mathrm{C}}(F, t)\left(1-\frac{1}{2}\left(\frac{F_{\mathrm{P}}}{F}\right)^{\frac{1}{2}}\right) .
$$

using the force scale $F_{\mathrm{P}}=\frac{k_{\mathrm{B}} T}{L_{\mathrm{P}}}$ with the persistence length $L_{\mathrm{P}}$. Accordingly Eq. 4.7 changes to

$$
F=k_{\text {eff }}\left(v t-L_{\mathrm{c}}\left(1-\frac{1}{2}\left(\frac{F_{\mathrm{P}}}{F}\right)^{\frac{1}{2}}\right)\right) .
$$

Eq. 4.9 is solved numerically. Fig. 4.5 shows a comparison of the resulting force-strain curve using Eqs. 4.7 and 4.9, respectively. Both curves are calculated using the most likely parameters obtained from the fit to the data (see below) at a loading rate of $v=$ $0.5 \mu \mathrm{m}$. Since $L_{\mathrm{K}} \approx 2 L_{\mathrm{P}}$, we used $F_{\mathrm{P}}=0.5 F_{\mathrm{K}}$. The curves show only minor deviations in the onset of the first linear regime at low strains. 
Chapter 4. Nonlinear Loading-Rate- Dependent Force Response of Individual

\subsubsection{Fit Results}

Each experimental force-time-curve is fitted independently with identical starting parameters, which are varied over multiple orders of magnitude. Based on the variance between the resulting parameters for different curves starting from the same parameters, we can distinguish which starting parameters lead to global minima conserved between different curves. The set of parameters with the lowest variance yields average potential widths $\bar{x}_{\mathrm{u}, 1}=0.09 \pm 0.04 \mathrm{~nm}, \bar{x}_{\mathrm{u}, 2}=0.12 \pm 0.04 \mathrm{~nm}$ and $\bar{x}_{\mathrm{u}, 3}=0.18 \pm 0.06 \mathrm{~nm}$. The average spring constant is found to be $\bar{k}_{\text {eff }}=0.22 \pm 0.06 \mathrm{pN} / \mathrm{nm}$. The most likely parameters, i.e. the maximum of the histograms shown in figure 4.6 and 4.7 , are $k_{\mathrm{eff}}^{*}=0.23 \mathrm{pN} / \mathrm{nm}, x_{\mathrm{u}, 1}^{*}=0.11 \mathrm{~nm}, x_{\mathrm{u}, 2}^{*}=0.13 \mathrm{~nm}$ and $x_{\mathrm{u}, 3}^{*}=0.15 \mathrm{~nm}$. The spread of the values is partly due to experimental and numerical uncertainties. There are, however, intrinsic sources of variance between individual filaments.

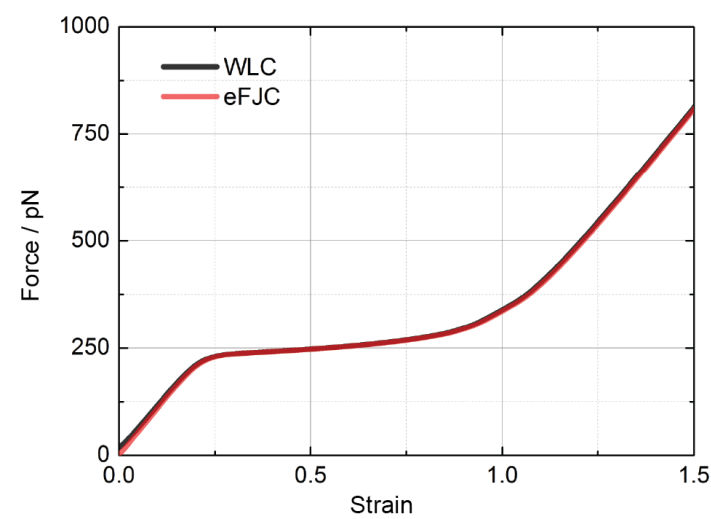

Figure 4.5: Comparison of WLC (black) and eFJC (red) models. 


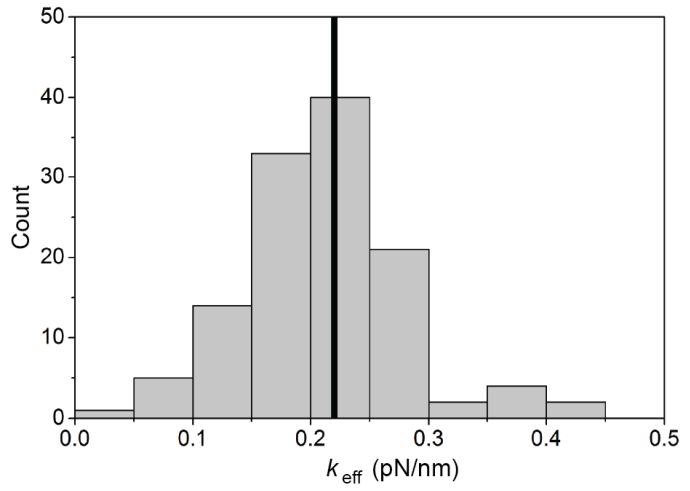

Figure 4.6: Fit results of $k_{\text {eff. }}$ The black line shows the average value of the distribution. 
Chapter 4. Nonlinear Loading-Rate- Dependent Force Response of Individual

a)

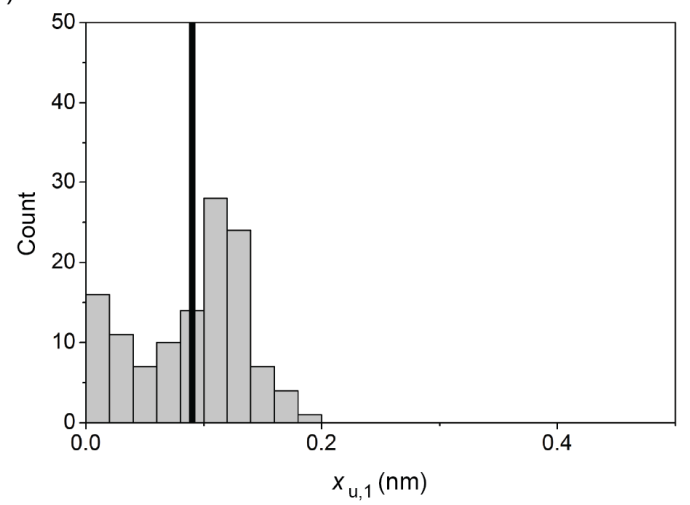

b)

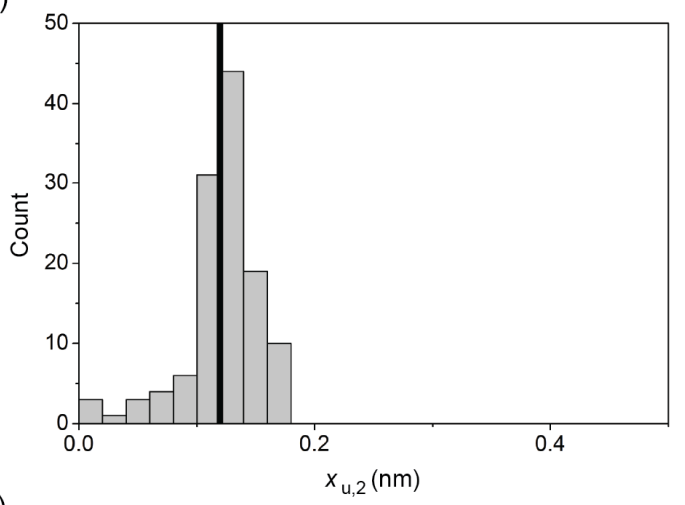

c)

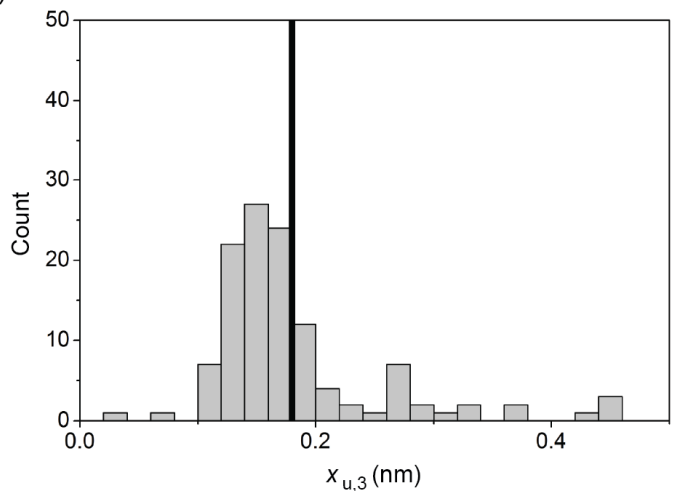

Figure 4.7: a) Fit results of $x_{u, 1}$, b) $\left.x_{u, 2}, c\right) x_{u, 3}$, with the average values of the distributions (black lines). 


\section{References}

1. Huber, F., Boire, A., López, M. P. \& Koenderink, G. H. Cytoskeletal Crosstalk: When Three Different Personalities Team Up. Curr. Opin. Cell Biol. 32C, 39-47 (2014).

2. Block, J., Schroeder, V., Pawelzyk, P., Willenbacher, N. \& Koster, S. Physical Properties of Cytoplasmic Intermediate Filaments. Biochim. Biophys. Acta 1853, 30533064 (2015).

3. Seltmann, K., Fritsch, A. W., Käs, J. A. \& Magin, T. M. Keratins Significantly Contribute to Cell Stiffness and Impact Invasive Behavior. P. Natl. Acad. Sci. USA 110, 18507-18512 (2013).

4. Ramms, L. et al. Keratins as the Main Component for the Mechanical Integrity of Keratinocytes. P. Natl. Acad. Sci. USA 110, 18513-18518 (2013).

5. Nicolet, S., Herrmann, H., Aebi, U. \& Strelkov, S. V. Atomic Structure of Vimentin Coil 2. J. Struct. Biol. 170, 369-376 (2010).

6. Chernyatina, A. A., Guzenko, D. \& Strelkov, S. V. Intermediate Filament Structure: the Bottom-Up Approach. Curr. Opin. Cell Biol. 32C, 65-72 (2015).

7. UniProt, last viewed: 2016.08 .05 (2016).

8. Kreplak, L. \& Fudge, D. Biomechanical Properties of Intermediate Filaments: from Tissues to Single Filaments and Back. Bioessays 29, 26-35 (2007).

9. Charrier, E. E. \& Janmey, P. A. Mechanical Properties of Intermediate Filament Proteins. Method. Enzymol. 568, 35-57 (2016).

10. Köster, S., Weitz, D. A., Goldman, R. D., Aebi, U. \& Herrmann, H. Intermediate Filament Mechanics in Vitro and in the Cell: From Coiled Coils to Filaments, Fibers and Networks. Curr. Opin. Cell Biol. 32C, 82-91 (2015).

11. Nöding, B. \& Köster, S. Intermediate Filaments in Small Configuration Spaces. Phys. Rev. Lett. 108, 088101 (2012).

12. Mücke, N. et al. Assessing the Flexibility of Intermediate Filaments by Atomic Force Microscopy. J. Mol. Biol. 335, 1241-1250 (2004).

13. Kreplak, L., Bär, H., Leterrier, J. F., Herrmann, H. \& Aebi, U. Exploring the Mechanical Behavior of Single Intermediate Filaments. J. Mol. Biol. 354, 569-577 (2005). 
Chapter 4. Nonlinear Loading-Rate- Dependent Force Response of Individual Vimentin Intermediate Filaments to Applied Strain

14. Kreplak, L., Herrmann, H. \& Aebi, U. Tensile Properties of Single Desmin Intermediate Filaments. Biophys J 94, 2790-2799 (2008).

15. Guzmán, C. et al. Exploring the Mechanical Properties of Single Vimentin Intermediate Filaments by Atomic Force Microscopy. J. Mol. Biol. 360, 623-630 (2006).

16. Qin, Z., Kreplak, L. \& Buehler, M. J. Hierarchical Structure Controls Nanomechanical Properties of Vimentin Intermediate Filaments. PLOS One 4, e7294 (2009).

17. Herrmann, H. \& Aebi, U. Intermediate Filaments: Molecular Structure, Assembly Mechanism, and Integration into Functionally Distinct Intracellular Scaffolds. Annu. Rev. Biochem. 73, 749-789 (2004).

18. Winheim, S. et al. Deconstructing the Late Phase of Vimentin Assembly by Total Internal Reflection Fluorescence Microscopy (TIRFM). PLOS One 6, e19202 (2011).

19. Mücke, N. et al. Molecular and Biophysical Characterization of Assembly-Starter Units of Human Vimentin. J. Mol. Biol. 340, 97-114 (2004).

20. Candelli, A., Wuite, Gijs J L \& Peterman, Erwin J G. Combining Optical Trapping, Fluorescence Microscopy and Micro-Fluidics for Single Molecule Studies of DNAProtein Interactions. Phys. Chem. Chem. Phys. 13, 7263-7272 (2011).

21. Heller, I. et al. STED Nanoscopy Combined with Optical Tweezers Reveals Protein Dynamics on Densely Covered DNA. Nat. Methods 10, 910-916 (2013).

22. See Supplemental Material at. http://link.aps.org/supplemental/10.1103/ PhysRevLett.118.048101 for a movie of the filament pulling, a more detailed description of the theoretical model, a comparison of the worm-like chain and freely jointed chain models and the fit results.

23. Savitzky, A. \& Golay, M. J. E. Smoothing and Differentiation of Data by Simplified Least Squares Procedures. Anal. Chem. 36, 1627-1639 (1964).

24. Barak, P. Smoothing and Differentiation by an Adaptive-Degree Polynomial Filter. Anal. Chem. 67, 2758-2762 (1995).

25. Qin, Z. \& Buehler, M. J. Structure and Dynamics of Human Vimentin Intermediate Filament Dimer and Tetramer in Explicit and Implicit Solvent Models. J. Mol. Model. 17, 37-48 (2011). 
26. Ackbarow, T. \& Buehler, M. J. Molecular Mechanics of Stutter Defects in Vimentin Intermediate Filaments. Exp. Mech. 49, 79-89 (2009).

27. Bendit, E. G. A Quantitative X-Ray Diffraction Study of the Alpha-Beta Transformation in Wool Keratin. Text. Res. J. 30, 547-555 (1960).

28. Rief, M. Elastically Coupled Two-Level Systems as a Model for Biopolymer Extensibility. Phys. Rev. Lett. 81, 4764-4767 (1998).

29. Friddle, R. W., Noy, A. \& de Yoreo, J. J. Interpreting the Widespread Nonlinear Force Spectra of Intermolecular Bonds. P. Natl. Acad. Sci. USA 109, 13573-13578 (2012).

30. Toan, N. M. \& Thirumalai, D. Theory of Biopolymer Stretching at High Forces. Macromolecules 43, 4394-4400 (2010).

31. Rief, M., Pascual, J., Saraste, M. \& Gaub, H. E. Single Molecule Force Spectroscopy of Spectrin Repeats. Low unfolding forces in helix bundles. J. Mol. Biol. 286, 553561 (1999).

32. Bell, G. Models for the Specific Adhesion of Cells to Cells. Science 200, 618-627 (1978).

33. Herrmann, H., Kreplak, L. \& Aebi, U. in Intermediate Filament Cytoskeleton (eds Omary, M. B. \& Coulombe, P. A.) Methods in Cell Biology v. 78, 3-24 (Elsevier Academic Press, Amsterdam and Boston, 2004). ISBN: 9780125641739. doi:10.1016/ S0091-679X (04)78001-2.

34. Ackbarow, T. \& Buehler, M. J. Superelasticity, Energy Dissipation and Strain Hardening of Vimentin Coiled-Coil Intermediate Filaments: Atomistic and Continuum Studies. J. Mater. Sci. 42, 8771-8787 (2007).

35. Strelkov, S. V. \& Burkhard, P. Analysis of Alpha-Helical Coiled Coils with the Program TWISTER Reveals a Structural Mechanism for Stutter Compensation. J. Struct. Biol. 137, 54-64 (2002).

36. Chernyatina, A. A., Nicolet, S., Aebi, U., Herrmann, H. \& Strelkov, S. V. Atomic Structure of the Vimentin Central Alpha-Helical Domain and Its Implications for Intermediate Filament Assembly. P. Natl. Acad. Sci. USA 109, 13620-13625 (2012). 
Chapter 4. Nonlinear Loading-Rate- Dependent Force Response of Individual Vimentin Intermediate Filaments to Applied Strain

37. Herrmann, H. et al. Structure and Assembly Properties of the Intermediate Filament Protein Vimentin: The Role of Its Head, Rod and Tail Domains. J. Mol. Biol. 264, 933-953 (1996).

38. Fudge, D. S., Gardner, K. H., Forsyth, V. T., Riekel, C. \& Gosline, J. M. The Mechanical Properties of Hydrated Intermediate Filaments: Insights from Hagfish Slime Threads. Biophys J 85, 2015-2027 (2003).

39. Kurniawan, N. A. et al. Fibrin Networks Support Recurring Mechanical Loads by Adapting Their Structure across Multiple Scales. Biophys J 111, 1026-1034 (2016).

40. Kroy, K. The Inelastic Hierarchy. Multiscale Biomechanics of Weak Bonds. Biophys J 111, 898-899 (2016).

41. Oliphant, T. E. Python for Scientific Computing. Comput. Sci. Eng. 9, 10-20 (2007).

42. Odijk, T. Stiff Chains and Filaments under Tension. Macromolecules 28, 70167018 (1995). 


\section{Chapter 5}

\section{Viscoelastic Properties of}

\section{Vimentin Originate from}

\section{Nonequilibrium Conformational}

\section{Changes}

This chapter is from "Viscoelastic Properties of Vimentin Originate from Nonequilibrium Conformational Changes" (J. Block et al., Science Advances, Vol. 4, No. 6, eaat1161, ISSN: 2375-2548, DOI: 10.1126/sciadv.aat1161). (C) The Authors, some rights reserved; exclusive licensee American Association for the Advancement of Science. Distributed under a Creative Commons Attribution NonCommercial License 4.0 (CC BY-NC)) http://creativecommons.org/licenses/by-nc/4.0/.

Johanna Block ${ }^{1, \star}$, Hannes Witt ${ }^{2, \star, \dagger}$, Andrea Candelli ${ }^{3,4}$, Jordi Cabanas Danes ${ }^{3,4}$, Erwin J. G. Peterman ${ }^{3}$, Gijs J. L. Wuite ${ }^{3}$, Andreas Janshoff ${ }^{2, \ddagger}$, Sarah Köster $^{1, \ddagger}$

${ }^{1}$ Institute for X-Ray Physics, University of Goettingen, 37077 Göttingen, Germany. 
Chapter 5. Viscoelastic Properties of Vimentin Originate from Nonequilibrium

${ }^{2}$ Institute of Physical Chemistry, University of Goettingen, 37077 Göttingen, Germany.

${ }^{3}$ Department of Physics and Astronomy and LaserLab, Vrije Universiteit Amsterdam, 1081 HV Amsterdam, Netherlands.

${ }^{4}$ LUMICKS B.V., 1081 HV Amsterdam, Netherlands.

‡ Corresponding author. Email: sarah.koester@phys.uni-goettingen.de (S.K.); andreas.janshoff@chemie.uni-goettingen.de (A.J.)

^ These authors contributed equally to this work.

${ }^{\dagger}$ Present address: Max Planck Institute for Dynamics and Self-Organization, Göttingen, Germany.

Contribution to the article: Vimentin has been produced and isolated by Susanne Bauch. I have prepared all buffers and the vimentin filaments for all experiments. I have also performed and optimized all the experiments. Hannes Witt and I both contributed to the data analysis. Hannes Witt developed and implemented the models and performed all simulations and model fittings. I produced all figures for the article. The first version of the manuscript has been written by Hannes Witt and myself. It was then iteratively optimized by Hannes Witt, Sarah Köster, Andreas Janshoff and myself. 


\subsection{Abstract}

Structure and dynamics of living matter rely on design principles fundamentally different from concepts of traditional material science. Specialized intracellular filaments in the cytoskeleton permit living systems to divide, migrate, and grow with a high degree of variability and durability. Among the three filament systems, microfilaments, microtubules, and intermediate filaments (IFs), the physical properties of IFs and their role in cellular mechanics are the least well understood. We use optical trapping of individual vimentin filaments to investigate energy dissipation, strain history dependence, and creep behavior of stretched filaments. By stochastic and numerical modeling, we link our experimental observations to the peculiar molecular architecture of IFs. We find that individual vimentin filaments display tensile memory and are able to dissipate more than $70 \%$ of the input energy. We attribute these phenomena to distinct nonequilibrium folding and unfolding of $\alpha$ helices in the vimentin monomers constituting the filaments.

\subsection{Introduction}

Vimentin belongs to the protein family of intermediate filaments (IFs), which, together with microfilaments (MFs) and microtubules (MTs), constitute the cytoskeleton of eukaryotic cells. It is widely accepted that this composite network of biopolymers plays an important role in cell mechanics, motility, adhesion, and contraction. Each filament type contributes distinct mechanical features; whereas MFs and MTs are well characterized from a physics point of view, IFs are less well understood. In contrast to actin and MTs, which are evolutionary highly conserved, IFs are encoded by 70 genes in humans [1], subdivided in five categories, and expressed in a cell-type-specific manner. Vimentin not only is the typical IF protein in cells of mesenchymal origin but is also overexpressed in cancer cells of epithelial origin and is associated with metastasis. Moreover, IFs have been proposed to play a major role in mechanical resistance and integrity of cells, especially in response to environmental stress[2-5].

All IFs share the secondary structure of the monomer (Fig. 5.1 A and Supporting Fig. 5.4), including an $\alpha$-helical rod domain and intrinsically disordered head and tail 
Chapter 5. Viscoelastic Properties of Vimentin Originate from Nonequilibrium

Conformational Changes

domains [6, 7]. The formation of extended filaments from cytoplasmic IF proteins follows a complex hierarchical scheme, distinct from MFs or MTs. First, lateral assembly of monomers via parallel coiled-coil dimers and half-staggered anti-parallel tetramers yields unit-length filaments (ULFs) [6], schematically shown in Fig. 5.1A (middle). Subsequently, longitudinal annealing of these ULFs results in mature, elongated filaments of $10 \mathrm{~nm}$ in diameter. This hierarchical filament formation via dimers, tetramers, octamers, and ULFs and the protofibrillar organization, which was found, for example, for keratin [8], are in stark contrast to MFs and MTs, which are built from globular subunits, and may be the reasons for the remarkable properties of IF. It should be noted that IFs, and in particular keratins, also form very stable and resistant natural superstructures such as wool and hair, whose overall mechanical resistance has been extensively studied since the beginning of the last century $[9,10]$. However, the properties of single cytoplasmic IFs, which are the basis for understanding how IFs provide cells with mechanical resistance, cannot be retrieved from these ensemble experiments.

Although IFs display intriguing mechanical characteristics [11-13], little is known about the mechanisms involved at the single filament and molecular levels. Stretching experiments and molecular dynamics simulations of IFs or subunits thereof have shown enormous extensibility of strains up to 3.5 [14-16], different stretching regimes $[14,17]$, and a loading-rate dependent force response [14]. Here, we directly probe the mechanical response of individual vimentin filaments to one-time and repeated stretching-relaxation cycles and to clamping at forces of 50 to $700 \mathrm{pN}$ [force clamp (FC)]. By theoretical modeling and Monte Carlo (MC) simulations based on structure predictions of IF subunits and the hierarchical assembly model, we directly link our experimental results to the molecular architecture of vimentin IFs. With this approach, we find strong evidence for intricate dissipative behavior and suggest a mechanism for the observed dependence of the mechanical properties on the strain history based on conformational switching between $\alpha$-helical and $\beta$ sheet secondary structures. The compiled data reveal how the molecular structure of the vimentin monomer imprints viscoelastic properties on larger length scales and show how extension far from equilibrium permits to dissipate a large amount of energy. We speculate that these concepts of controlled failure due to weak transient bonds and reversible conformational changes are important to protect cells in situations of extreme stresses. From a physics point of 
view, our observations give rise to single filament viscoelasticity based on nonequilibrium transformation of the secondary and tertiary protein structure.
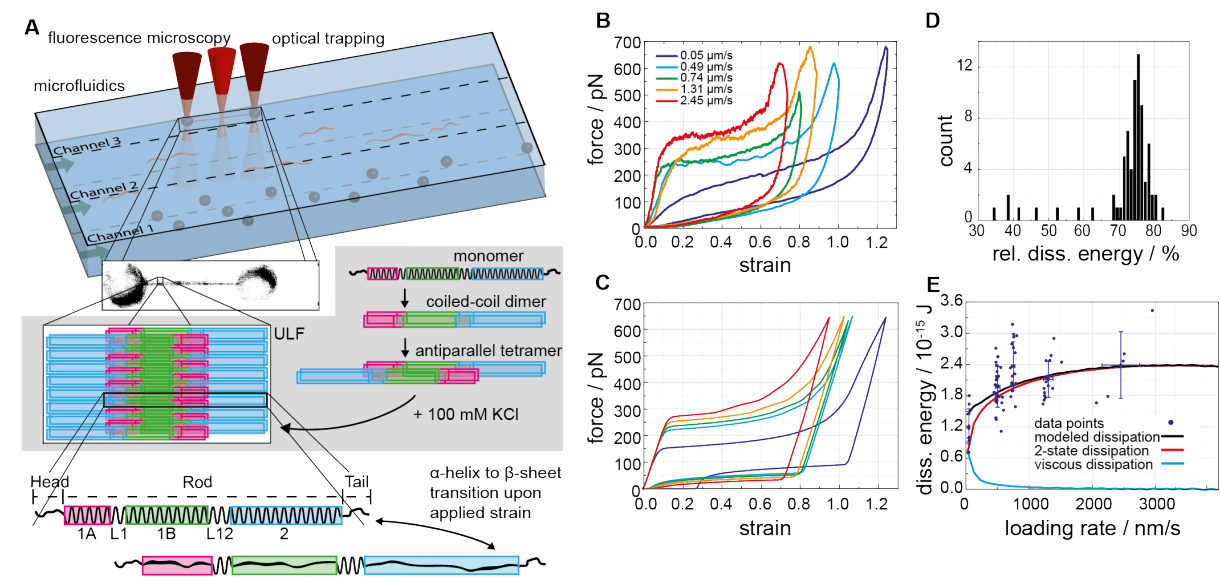

Figure 5.1: Experimental setup, vimentin assembly and comparison of effective two-state model and experimental data. (A) Top: Microfluidic chip. Middle: Schematic representation of vimentin assembly. Bottom: Secondary structure of the IF monomer; $\alpha$-helical rod, subdivided into the three $\alpha$ helices, $1 A, 1 B$ and 2, and intrinsically disordered head and tail domains. (B) Force-strain cycles of single vimentin filaments at different loading rates. (C) Force-strain cycles for different loading rates [color-coded as in (B)] calculated by the effective two-state model. (D) Dissipated energy during individual stretching cycles. (E) Comparison between energy dissipation measured for 75 different filaments at five different velocities between 50 and $2450 \mathrm{~nm} / \mathrm{s}$ (dark blue dots) and prediction by the two-state model (black); nonequilibrium transition between $\alpha$ helices and $\beta$ sheets (red); viscous dissipation (light blue). Error bars indicate SD.

\subsection{Results}

\subsubsection{Pronounced Energy Dissipation}

To elucidate the energy dissipation mechanisms in vimentin filaments, single filaments were stretched up to forces of 500 to $600 \mathrm{pN}$ and subsequently relaxed (Fig. 5.1 B). Loading and unloading were performed at different velocities varying over two orders of magnitude. The extension curves show three regimes: a linear increase, a plateau (presumably caused by unfolding of $\alpha$ helices in the vimentin monomers, the so-called $\alpha$ - $\beta$ transition), and a second linear increase with strain [14, 17]. In contrast, the relax- 
ation curves do not exhibit a force plateau and are largely independent of the relaxation velocity, indicating a strong asymmetry of the free-energy potential of the $\alpha$ - $\beta$ transition [18]. A striking feature of the force-strain cycles is the pronounced hysteresis between the extension and the relaxation curves. The area between these two curves equals the energy uptake of the filament during one stretching-relaxation cycle and is on the order of several femtojoules for a single filament. The energy uptake may not only be directly dissipated but could also be stored in the altered conformation of the filament as potential energy. Since these processes cannot be distinguished easily, we refer to the area between the two curves as apparent dissipation.

We have recently shown [14] that the experimental force-strain curves can be accurately replicated by a model that was first proposed by Burte and Halsey [10] for the elongation of wool fibers and among others used by Rief et al. [18] and Minin et al. [19]. In this model, we forego the parallel monomers and model each ULF as a single effective element, which consists of three helical domains (1A, 1B, and 2) that may switch between a short and an elongated conformation depending on the applied force, corresponding to the $\alpha$-helical or the $\beta$ sheet conformation, respectively. For simplicity, we refer to this approach as an effective two-state model, being aware that it actually comprises a coupled system of multiple two-state elements. Although the model is not able to capture every aspect of vimentin stretching, it provides a good description of initially, once-only, stretched filaments and their relaxation [14]. Since all elements start in the $\alpha$ state and all parallel monomers switch in a rapid reaction cascade to the $\beta$ state as they are dilated, they can be mapped on a single effective element. Another proposed mode of elongation for IFs is sliding of the subunits past each other [14, 17], which probably leads to viscous behavior and is introduced into the model by adding an idealized viscous dash pot in series. The effective two-state model is able to quantitatively reproduce the experimental force cycles (Fig. 5.1, B and C). The main difference between the experimental curves and the corresponding curves obtained from the effective two-state model is the shape of the retraction curve: For the relaxation data, while the two-state model shows a sharp edge at the onset of $\alpha$ helix formation, the experimental curve is more convex, in particular for the slowest loading rate. We may attribute this effect to lowering of the persistence length upon stretching (fig. 5.5). The reduction in persistence length might arise from the thinning of the filament [16], from 
the decoupling of parallel monomers, or from a reduction of the persistence length of $\beta$ sheets compared to $\alpha$ helices [20].

The apparent energy dissipation comprises more than $70 \%$ of the total energy (Fig. $5.1 \mathrm{D})$ put into the filament, which is comparable to biological high-performance materials such as fibrin fibers, spider silk or titin [20-24]. One might be tempted to attribute the apparent energy dissipation to viscous friction during sliding of IF subunits against each other. However, our analysis indicates that the major contribution to the energy dissipation originates from the unfolding and refolding of the $\alpha$ helices occurring far from equilibrium. Nonequilibrium conformational changes have been identified as a mechanism for energy dissipation early on and can even be considered as an origin of viscous behavior, in contrast to the prevalent "exchange of old neighbors for new ones"-concept of viscosity [10]. For the sake of clarity, we will refer to the former as nonequilibrium unfolding and to the latter as viscosity. We compare the contributions of the two different mechanisms to the apparent energy dissipation by switching off the viscous element in the model (light blue curve in Fig. 5.1 E). Surprisingly, we find that the apparent energy dissipation is indeed dominated by nonequilibrium unfolding (red curve), while viscous contributions only become relevant at low loading and relaxation rates. Whether this viscous contribution is due to sliding of subunits or due to the extension of head and tail domains in the filament is not possible to reveal from our current data.

\subsubsection{A Biopolymer with Tensile Memory}

To address the question of filament adaption to mechanical stress, we performed repeated stretching cycles to increasing distances with individual vimentin filaments (Fig. 5.2 A, and MC simulations in Fig. 5.2 B) and found a strong dependency on the strain history, which we refer to as tensile memory. The initial slope of the force-strain curve, and therefore the stiffness of the filaments (Fig. 5.2 C), considerably decreases from cycle to cycle. The filaments reach their initial length after each full cycle. This complex tensile behavior cannot be explained by the effective two-state model but requires that each element is able to adopt additional states [10]. Therefore, we devise a stochastic model explicitly considering that the filaments consist of multiple (32 on average [6]) monomers per cross-section. We assume that, during relaxation of the fil- 

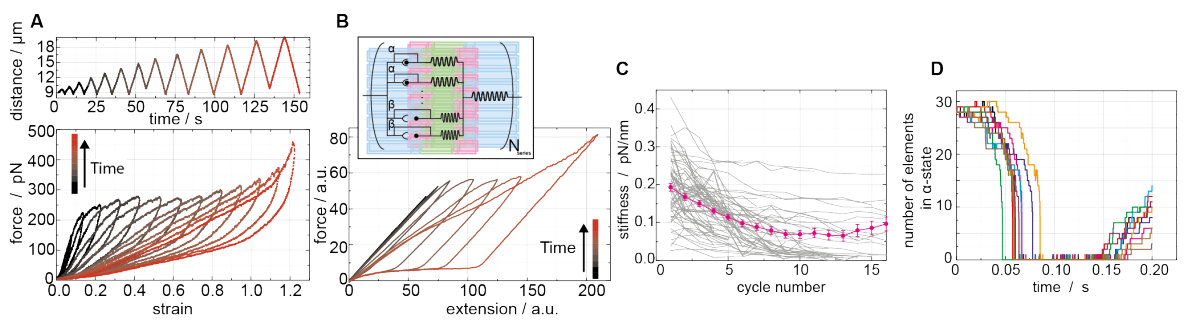

Figure 5.2: Response to repeated strain. (A) Top: Distance versus time. Bottom: Force versus strain for a typical filament. (B) Kinetic MC simulations of a simplified vimentin filament that take into account 32 monomers per cross-section (inset) reproduce a curve progression resembling the experiment. The force and extension in (B) are given in arbitrary units (a.u.) of force and distance. (C) Fitting the linear regime of the stretching part of every cycle [(A); up to $10 \%$ strain] yields the stiffness for each cycle. The progression with cycle number for 77 different filaments is shown. Magenta, average stiffness per cycle. Error bars indicate SEM. (D) Every monomer within the simulated IF is traced by the MC simulation. Transition of the $32 \alpha$ helices of eight individual ULFs (color-coded) within one filament.

ament, not all parallel monomers return to the $\alpha$ state but some remain in the $\beta$ state. The length of the ULF is set by the length of the shortest element, explaining why the filament reaches its initial length after each cycle. For this reason, only the shorter elements experience the applied force upon restretching; therefore these elements determine the filaments' stiffness, which is approximately proportional to the number of parallel $\alpha$ helices. After each force cycle, an increasing number of helices remain in the $\beta$ state, therefore lowering the stiffness of the filament with each cycle. A similar mechanism has been proposed for fibrin and collagen networks, where elongation of individual fibers was identified as the origin of the observed tensile memory [25]. We performed MC simulations of an idealized vimentin model consisting of 100 ULFs in series (Fig. 5.2 B, inset, fig. 5.6), each containing 32 parallel elements. The simulation illustrates that the model qualitatively reproduces the experimentally observed tensile memory.

Figure 5.2D shows tracking of the numbers of parallel elements in the $\alpha$ state in eight typical MC-modeled ULFs during stretching. In the beginning of the stretching cycle, the number of closed, $\alpha$-helical elements stays almost constant until all parallel elements in a ULF unfold rapidly in a reaction cascade, justifying the mapping of those parallel monomers on a single element in the two-state model. In contrast, during the 
relaxation of the filament, the number of elements in the $\alpha$ state in a ULF increases at a steady rate because they do not experience the force acting on the filament, leading to an incomplete refolding of ULFs at high rates, which, in turn, causes the observed softening. In unstressed vimentin monomers, the $\alpha$ helix is the most stable secondary structure with a lower energy compared to the $\beta$ sheet conformation. Therefore, a part of the energy uptake during stretching, referred to here as apparent dissipation, is stored in the filament as potential energy if monomers remain in the $\beta$ state after stretching and relaxing. The stored energy is dissipated when the stress is relieved from the filament, since eventually all elements return to the $\alpha$-helical conformation. Thus, the parallel architecture of vimentin leads to increased apparent dissipation.

\subsubsection{Two Modes of Filament Elongation}

To gain deeper insights in the different mechanisms of vimentin elongation, we additionally performed constant force experiments at different forces from 50 to $700 \mathrm{pN}$. Representative FC curves at 500 and $50 \mathrm{pN}$ are shown in Fig. 5.3 (A and B), respectively. The data of all individual curves are presented in a double-logarithmic plot in Fig. 3C. We observe two distinct regimes in filament behavior: a stepwise lengthening at low forces (50 and $100 \mathrm{pN}$ ) and a creep-dominated elongation at high forces (500 and $700 \mathrm{pN}$ ), with an intermediate regime at $250 \mathrm{pN}$ (Fig. 5.3C). We still observe steps even at higher forces, but the percentage of lengthening due to steps is reduced compared to lower forces. A histogram of step sizes shows a most likely step size of $10 \mathrm{~nm}$ independent of the applied force (Fig. 5.3 D, fig. 5.7). Additional FC experiments with maleimide-coupling between filament and beads (fig. 5.8) and relaxation experiments without force-feedback showing improved step height resolution (fig. 5.9 and 5.10) confirm that the steps are intrinsic to the filaments.

The observation of discrete elongation steps in vimentin filament agrees with the hypothesis that $\alpha-\beta$ transitions in the monomers are the main mechanism for vimentin elongation and dissipation at low strains. For full unfolding of one of the three $\alpha$-helical domains, one would expect a length change between $7.2 \mathrm{~nm}$ for the unfolding of domain $1 \mathrm{~A}$ and $27.8 \mathrm{~nm}$ for domain 2 [7, 14, 26]. This agrees well with the experimentally observed step sizes. Under FC conditions, the two-state model can be solved analytically. The model predictions for a typical filament length of $10 \mu \mathrm{m}$ are shown in 

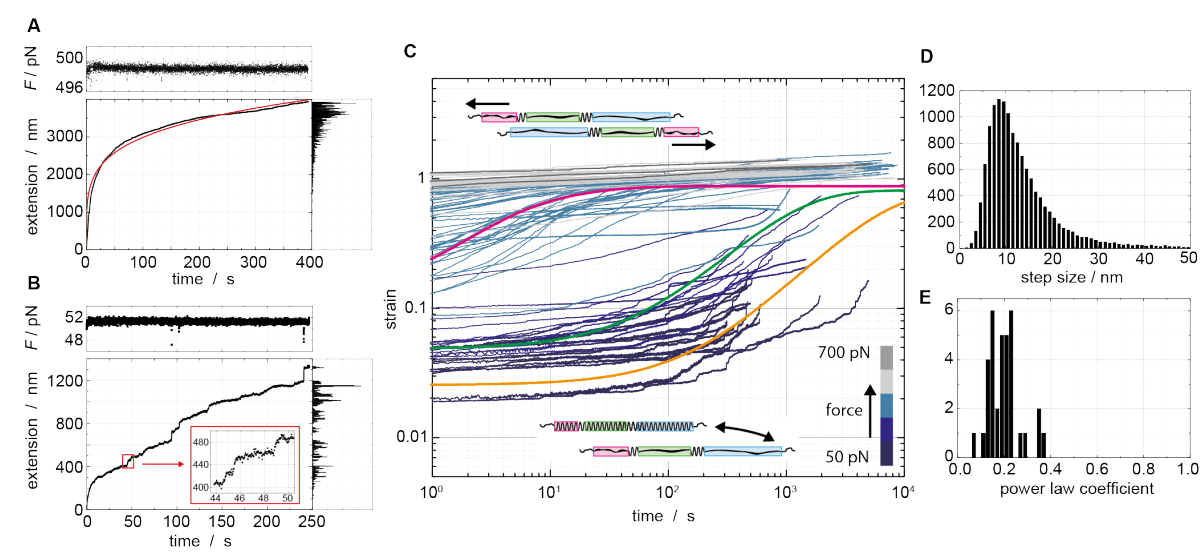

Figure 5.3: Response of individual vimentin IFs to applied constant force. (A and B) Examples of single vimentin filaments responding to a constant force (FC) of 500 and $50 \mathrm{pN}$, respectively. Top: Constant force versus time. Bottom: Filament extension versus time. Right: Histogram of filament length; power-law fit (red line). (C) Log-log plot of the filament strain versus time for FC experiments on about 100 vimentin filaments at different forces from 50 to $700 \mathrm{pN}$. The three single curves in orange, green and magenta show the prediction of the two-state model for a filament responding to a constant force of 50, 100 and $250 \mathrm{pN}$, respectively. The cartoons illustrate the two elongation mechanisms: Top: Viscous sliding. Bottom: $\alpha-\beta$ transition. (D) Histogram of all steps. (E) Power-law coefficients found for filaments at 500- and 700- $p N$ FCs.

Fig. 5.3C for FCs at 50, 100 and $250 \mathrm{pN}$ as orange, green and magenta lines, respectively, and agree well with the experimental observations.

FCs at forces larger than the plateau force show a qualitatively different progression than at lower forces (Fig. 5.3C). The elongation is dominated by creep (Fig. 5.3A) and the time-dependent change in length can be well described by a power-law [27]. Powerlaw behavior is indicative of viscoelasticity with a broad distribution of relaxation times. The power-law coefficient $b$ is a measure of the system's viscosity with a coefficient between 0 and 1 for purely elastic to purely viscous systems, respectively. Here, we find power-law coefficients of 0.2 (Fig. 5.3E), which are typically found for living cells when discussed in the framework of soft-glassy materials $[27,28]$. In vitro vimentin networks were found to display mostly elastic behavior dominated by the filaments' persistence length, the networks' mesh size, and the concentration of cross-linking ions [21, 29-31]. It is important to note that the power-law viscosity observed in the present work is only observable at large strains and large prestresses and should not be linked to rheological 
data of vimentin networks that fulfill a linear stress-strain relationship.

The physical picture behind power-law rheology describes the material as discrete elements aggregated by weak interactions, which lead to an energy landscape of multiple wells with different depths [27]. Here, each element is a vimentin subunit, forming a filament by weak interactions. In this picture, the observed viscous flow equals sliding of vimentin subunits, for example, the dimers moving against each other and thereby transferring momentum, as proposed by molecular dynamics simulations of the vimentin tetramer [17].

\subsection{Discussion}

Vimentin has remarkable mechanical properties, such as tensile memory, an enormous degree of energy dissipation upon stretching, mostly due to nonequilibrium $\alpha$ - $\beta$ transitions, and high durability sustaining stresses of several megapascals, properties otherwise only observed in high-performance biomaterials like fibrin/fibrinogen or spider silk [20-24]. The mechanical features of vimentin filaments are most distinct at high strains and large loads of several hundreds of piconewton. This finding raises the following questions: To what extent are these properties of physiological relevance? In particular, are IFs in the cytoskeleton are under tensile load and do cells experience and survive strains of that magnitude? Fudge et al. [32] found not only that keratinocytes survived strains of $138 \%$ but also that large strains cause straightening of keratin IFs, confirming tensile load, and lead to elongation of IFs. Although there are no direct observations of IFs within cells stretched to large strains in vivo, the strain resilience of cells observed in vitro supports a physiological relevance of the strains applied here.

The use of two different levels of mechanical modeling, a stochastic model explicitly taking the parallel monomers into account and a coarser numerical effective two-state model foregoing parallel elements, allowed us to relate our experimental observations to molecular processes. Both models are limited in their application. The numerical two-state model fails to reproduce all phenomena related to parallel elements in different folding states, such as the tensile memory, and can therefore only be applied to initially relaxed filaments. However, in this case, the model is suitable to quantitatively reproduce the nonlinear force response and the energy dissipation during vimentin elon- 
gation. By contrast, the stochastic parallel element model is computationally much more demanding, rendering any attempt of direct data fitting unfeasible, and is difficult to combine with additional elements such as viscous contributions. Nevertheless, both models are consistent with each other and reflect well how the hierarchical organization of the vimentin filament affects its overall mechanical behavior. Both the tensile memory and the pronounced energy dissipation can be related to vimentin's architecture of parallel monomers. In our analysis, ULFs partially in $\alpha$-helical and partially in $\beta$ sheet configuration play a crucial role by allowing a change in the filament's stiffness without altering its length.

We were able to demonstrate that the observed energy dissipation can be mostly explained by nonequilibrium $\alpha$ - $\beta$ transitions in the vimentin monomer. This reaction has been observed early on in strained wool fibers [9] and, more recently, was also directly measured in an engineered vimentin superstructure using wide-angle x-ray scattering [33]. Simulations of strained vimentin dimers and tetramers illustrate how this transition occurs on the molecular level [17]. While it is so far not possible to directly observe this conformational change at the level of single filaments, the good agreement between the experimentally recorded force-distance curves and the modeled curves based on this known conformational transition support the computational prediction [14]. The strategy to use a nonequilibrium transition providing additional length to the vimentin filament to maximize energy dissipation is fundamentally the same mechanism as observed for spider silk, titin or fibrin [20-24], albeit using the more subtle $\alpha$ - $\beta$ transition compared to the unfolding of globular protein domains in titin and fibrin or $\beta$ sheet crystals in silk.

Because of their low persistence length compared to MFs and MTs, IFs were often assumed to be of minor relevance for cell mechanics. However, the pronounced energy dissipation observed here might indicate that IFs act as intracellular shock absorbers against tensile stresses, as they are able to efficiently dissipate large amounts of mechanical energy, thereby protecting the cellular integrity. This agrees with the observation that the effect of a keratin knockout in keratinocytes on the mechanical properties is much more pronounced when the cells are subjected to tensile stresses compared to compressive loading [2, 3].

We found that individual vimentin filaments get softer upon repeated stretching, 
whereas the filaments' length is not influenced by the strain history. While a similar behavior was observed in networks composed of fibrin or collagen [25], vimentin behavior is notably distinct from the observations for single fibrin [25] or titin [34] fibers, which show a change in length upon repeated stretching cycles while maintaining their mechanical properties. This behavior is consistent with our proposed mechanism based on the supramolecular architecture of vimentin filaments using numerous highly ordered parallel monomers in contrast to both titin and fibrin.

Vimentin is found in cells of mesenchymal origin that are exposed to relevant stresses, and the ability to dissipate large amounts of energy may help prevent mechanical damages. For example, the endothelium and smooth muscles form the intima and the media of blood vessels. The tensile memory could potentially help these cells to be compliant with the beating of the heart and the repeated pressure shift between systolic and diastolic blood pressure. This hypothesis is supported by the observation that vimentin expression is elevated in cardiovascular segments subjected to high blood pressure such as the pulmonary trunk, the aorta and the left ventricle [35].

To conclude, our data reveal how the molecular structure of the vimentin monomer imparts viscoelastic properties at larger length scales and show how extension far from equilibrium permits the dissipate of a large amount of energy. We were able to directly observe step-wise elongation of vimentin filaments attributed to $\alpha$ - $\beta$ transitions in the protein monomers. The ULF architecture of parallel extensible monomers permits the establishment of tensile memory at repeated load, while sliding of the filaments gives rise to a power-law viscoelasticity at large strain. Thus, our results explain the intriguing mechanical properties of vimentin IFs, such as extreme extensibility, tensile memory and a nonequilibrium mechanism for energy dissipation by the particular molecular architecture of IF reliance on weak bonds that display controlled and reversible failure in the filaments. 


\subsection{Material and Methods}

\subsubsection{Vimentin Expression and Purification Protocol}

Escherichia Coli T61 cells (\#3017, Zymo Research) were used to recombinantly express human vimentin C328A with additional amino acids GGC at the C-terminus (plasmid DNA from H. Herrmann, German Cancer Research Center). The protocol was adopted from Herrmann et al. [36] One hundered microliters of thawed bacteria solution and $1 \mu \mathrm{l}$ plasmid solution $(1 \mu \mathrm{g} / \mu \mathrm{l}$; diluted 1:100) were mixed and incubated on ice for $5 \mathrm{~min}$. Fifty microliters of this mixture was plated on an ampicillin-containing lysogeny broth agar plate (L5667, Sigma-Aldrich) and incubated at $37^{\circ} \mathrm{C}$ overnight.

Single bacteria colonies were picked, transferred into $50 \mathrm{~mL}$ terrific broth (TB; 47.6 g/liter; \#T0918, Sigma-Aldrich) medium containing glycerol (10-11 g/liter) and ampicillin $\left(0.1 \mathrm{mg} / \mathrm{ml}\right.$; Sigma-Aldrich), and incubated at $37^{\circ} \mathrm{C}$ and $150 \mathrm{rpm}$. After 6 hours, the bacteria solution was transferred to 1 liter fresh TB medium containing ampicillin $(0.1 \mathrm{mg} / \mathrm{ml})$ and was allowed to grow at $37^{\circ} \mathrm{C}$ and $150 \mathrm{rpm}$ overnight.

Protein preparation started by pelleting bacteria with a Beckman centrifuge (Beckmann Coulter Avanti J-26 XP, rotor JS5.3) at $5000 \mathrm{~g}$ for $15 \mathrm{~min}$ at $4{ }^{\circ} \mathrm{C}$. During the subsequent steps, the protein was always kept on ice. The pellet was transferred to a cooled douncer, homogenized with $16 \mathrm{ml}$ of tris buffer $(50 \mathrm{mM}$; pH 8.0; Carl-Roth $\mathrm{GmbH}$ ) containing saccharose $(25 \%$, w/v), EDTA ( $1 \mathrm{mM}$, Carl-Roth $\mathrm{GmbH})$, and lysozyme $(10 \mathrm{mg} / \mathrm{mL}$, Roche Diagnostics), and incubated on ice for $30 \mathrm{~min}$. Eight hundred microliters of $\mathrm{MgCl}_{2}$ solution ( $1 \mathrm{M}$, Sigma-Aldrich), $80 \mu \mathrm{l}$ of deoxyribonuclease I (DNase 1; $50 \mathrm{mg} / \mathrm{ml}$; Sigma-Aldrich) in tris buffer (10 mM; pH 7.5) containing $\mathrm{NaCl}(100 \mathrm{mM}$; Carl-Roth $\mathrm{GmbH}$ ), $80 \mu \mathrm{l}$ ribonuclease A (RNase A; $10 \mathrm{mg} / \mathrm{ml}$; Roche Diagnostics) in tris buffer (100 mM; pH 7.5), $800 \mu$ l saturated phenylmethylsulfonyl fluoride (PMSF; Serva) in ethanol, and $1.6 \mathrm{ml}$ of $10 \%$ NP-40 (Roche Diagnostics) were added and mixed several times by homogenization. Forty milliliters of tris buffer $(20 \mathrm{mM}$; pH 7.5), containing $\mathrm{NaCl}(200 \mathrm{mM}), \mathrm{NP}-40(1 \%)$, EDTA $(2 \mathrm{mM})$, and $3 \alpha, 12 \alpha$-di-hydroxy- $5 \beta$-cholanic acid sodium salt (1\%; Sigma-Aldrich), was complemented by $800 \mu \mathrm{l}$ of a $1 \mathrm{M}$ 1,4-dithiothreitol solution (DTT; Carl-Roth GmbH), $1.2 \mathrm{ml}$ PMSF and $400 \mu \mathrm{l}$ of a $50 \mathrm{mM}$ Pefabloc SC solution (Carl-Roth $\mathrm{GmbH}$ ), and added to the douncer/bacteria solution. The mixture was incubated for $10 \mathrm{~min}$, transferred to Nalgene centrifuge bottles (250- 
mL PPCO-centrifuge bottles with sealing closure, Thermo Fisher Scientific) and centrifuged for $25 \mathrm{~min}$ at $10,000 \mathrm{~g}$ and $4{ }^{\circ} \mathrm{C}$ using a Beckman centrifuge (Beckman Coulter Avanti J-26 XP, rotor JLA 16.250). The supernatant was discarded, and the pellet was washed by homogenization in the douncer using washing buffer [ $80 \mathrm{ml}$ of tris $(10 \mathrm{mM}$; pH 8.0) containing Triton X-100 (0.5\%, v/v; Carl-Roth GmbH) and EDTA (5 mM)] complemented by $80 \mu \mathrm{l}$ of a 1 M DTT solution, $400 \mu \mathrm{l}$ of PMSF, and $200 \mu \mathrm{l}$ of a $50 \mathrm{mM}$ Pefabloc SC solution. The mixture was incubated $10 \mathrm{~min}$ on ice and centrifuged using the same conditions as for the previous step. Supernatant was again discarded, and the pellet was washed using $80 \mathrm{ml}$ of washing buffer containing $\mathrm{KCl}(1.5 \mathrm{M})$ complemented by $80 \mu \mathrm{l}$ of a 1 M DTT solution, $400 \mu \mathrm{l}$ of PMSF, and $200 \mu \mathrm{l}$ of a $50 \mathrm{mM}$ Pefabloc SC solution. After homogenization, the mixture was incubated for $30 \mathrm{~min}$ before centrifugation, which was performed as before. The third washing step was the exact repeat of the first washing step. For the last washing step, $80 \mathrm{ml}$ tris $(10 \mathrm{mM}$; pH 8.0), containing EDTA $(0.1 \mathrm{mM})$ complemented by $80 \mu \mathrm{l}$ of a 1 M DTT solution, $400 \mu \mathrm{l}$ of PMSF and $200 \mu \mathrm{l}$ of a $50 \mathrm{mM}$ Pefabloc SC solution, was used. As before, the pellet was washed and incubated for $10 \mathrm{~min}$, and the mixture was centrifuged. The supernatant was discarded, and the pellet was transferred to a douncer, warmed to room temperature, and solubilized in urea solution $(9.5 \mathrm{M})$. The recipe for the urea solution was as follows: $7.125 \mathrm{ml}$ urea (9.5 M; Carl-Roth $\mathrm{GmbH}$ ), $75 \mu \mathrm{l}$ of $1 \mathrm{M}$ tris ( $\mathrm{pH} 7.5$ ), $75 \mu \mathrm{l}$ of $0.5 \mathrm{M}$ EDTA, $75 \mu \mathrm{l}$ of PMFS and $75 \mu \mathrm{l}$ of $1 \mathrm{M}$ DTT. To yield a high protein concentration, as little of the urea solution as possible was used to dissolve the pellet. The solution was homogenized and finally centrifuged using a Beckman ultracentrifuge (Beckman Coulter Optima L90K, rotor Ti70) at $20^{\circ} \mathrm{C}$ and $100,000 \mathrm{~g}$ for $60 \mathrm{~min}$. The vimentin-containing supernatant was transferred to a Falcon tube, and methylamine hydrochloride solution (MAC; Sigma-Aldrich) was added to a final concentration of $10 \mathrm{mM}$.

For purification from the supernatant, an anion exchange chromatography step was followed by a cation exchange chromatography step. For anion exchange chromatography, a chromatography column (inner diameter, $15 \mathrm{~mm}$; length, $200 \mathrm{~mm}$; volume, $35 \mathrm{ml}$; \#7317-1522; BioRad) was prepared according to the user's manual. As column material, $10 \mathrm{ml}$ of DEAE sepharose (GE Healthcare) was mixed with $10 \mathrm{ml}$ of column buffer $[400 \mathrm{ml}$ of urea $(10 \mathrm{M}), 50 \mathrm{ml}$ of $50 \mathrm{mM}$ tris (pH 8.0), containing EDTA (10 mM) and EGTA ( $1 \mathrm{mM}$ ), filled to $500 \mathrm{ml}$ with MilliQ water and complemented by $500 \mu \mathrm{l}$ of 
Chapter 5. Viscoelastic Properties of Vimentin Originate from Nonequilibrium

$1 \mathrm{M}$ DTT] [a final pH of 7.5 was adjusted by hydrochloric acid (Carl-Roth GmbH)]. A $\mathrm{KCl}$ gradient was used to elute the protein. To do so, a gradient mixer $(2 \times 50 \mathrm{ml}$; Schütt 24) was filled with $40 \mathrm{ml}$ of column buffer in the front chamber and $40 \mathrm{ml}$ of column buffer containing $0.896 \mathrm{~g} \mathrm{KCl}$ (Carl-Roth $\mathrm{GmbH}$ ) in the rear chamber. When the sample entered the column, it was washed with two bed volumes of column buffer using a slow flow velocity. When the collected flow-through was equal to the dead volume of the column, the $\mathrm{KCl}$ gradient was started, and sample collection began (fractioning in $1.5 \mathrm{~mL}$ tubes). The protein concentration was monitored by measuring the absorption at $280 \mathrm{~nm}$ (NanoDrop, ND-1000, Thermo Fisher Scientific).

As an additional quality control, an $8 \%$ SDS gel was performed using samples collected after each purification step and from fractions of interest after chromatography. Fractions with high protein concentration after the DEAE column were pooled for a cation exchange chromatography, which was performed in analogy to the anion exchange chromatography but with CM Sepharose (GE Healthcare) as column material. In addition, the rear chamber was filled with $40 \mathrm{ml}$ of column buffer containing $1.792 \mathrm{~g}$ $\mathrm{KCl}$. The protein concentration of collected liquid was again monitored by measuring the absorption at $280 \mathrm{~nm}$ and fractions with high protein concentration were pooled. Lastly, MAC was added to the vimentin solution to a final concentration of $10 \mathrm{mM}$. Aliquots of the protein solution were stored at $-80^{\circ} \mathrm{C}$.

\subsubsection{Vimentin Labeling}

For fluorescence imaging of vimentin filaments and binding to streptavidin-coated beads, the protein was labeled with ATTO647N-maleimide (AttoTech $\mathrm{GmbH}$ ) and biotin-maleimide (Jena Bioscience $\mathrm{GmbH}$ ), respectively. Labeling was performed as described by Block et al. [14] and Winheim et al. [37].

\subsubsection{Vimentin Reconstruction and Assembly}

Labeled and unlabeled vimentin was stored in $8 \mathrm{M}$ urea at $-80^{\circ} \mathrm{C}$. By step wise dialysis $(8,6,4,2,1,0 \mathrm{M}$ urea) into phosphate buffer [2 mM, pH 7.5 disodium hydrogen phosphate (Sigma Aldrich) and sodium dihydrogen phosphate (Sigma Aldrich)], vimentin renaturation and dimer and tetramer formation were initiated. For dialysis, unlabeled 
and labeled vimentin was mixed in the desired ratio and transferred to 50-kDa cutoff dialysis tubing (SpectraPor, Carl-Roth $\mathrm{GmbH}$ ). Each dialysis step had a duration of $30 \mathrm{~min}$. Lastly, the dialysis tubing containing the vimentin was placed in $2 \mathrm{mM}$ phosphate buffer for $2 \mathrm{~h}$ at room temperature or at $10^{\circ} \mathrm{C}$ overnight.

Filament formation was initiated by $\mathrm{KCl}$. To do so, the vimentin concentration was adjusted to $0.2 \mathrm{~g} /$ liter, monitored by ultraviolet-visible spectrometry, by adding phosphate buffer. Afterwards, vimentin was placed in a fresh piece of 50-kDa cutoff dialysis tubing, placed in phosphate buffer ( $2 \mathrm{mM}$; pH 7.5), containing $\mathrm{KCl}(100 \mathrm{mM})$, and kept at $37^{\circ} \mathrm{C}$ overnight. This dialysis procedure ensured filaments that were homogeneous in diameter [6]. Assembled filaments were diluted 1:10 with $\mathrm{KCl}$-containing phosphate buffer and kept at $4{ }^{\circ} \mathrm{C}$ or on ice until further use. The morphology of the assembled filaments was monitored by epifluorescence microscopy, atomic force microscopy, end electron microscopy and found to be the typical morphology of IFs (see fig. 5.12).

\subsubsection{Maleimide Functionalization of Beads}

Functionalization of carboxylated polystyrene beads $(4.0-4-4 \mu \mathrm{m} ; 5 \% \mathrm{w} / \mathrm{v}$; PPs4.2COOH; Kisker biotech $\mathrm{GmbH} \&$ Co.KG) was performed according to Janissen et al. [38]. Beads were coated using maleimide-PEG-NH2 (molecular weight 5000 Da; PG2-AMM2-5k, Scholz Labor- und Klinikversorgungs GmbH), NH2 -PEG-OH (IrisBiotech) and 1-ethyl-3-(3-dimethyl-amino-propyl)-carboxiimide (E1769-1G, SigmaAldrich). Functionalized beads were stored in 1x phosphate-buffered saline (lot no. 73604799A, Invitrogen AG) containing $2 \%$ bovine serum albumin (A7906-10G, lot no. SLBB476V, Sigma-Aldrich).

\subsubsection{Experimental setup and procedure}

Vimentin force-extension experiments were performed using instruments combining dual optical tweezers (for manipulation) and fluorescence microscopy (for visualization). Most experiments were performed on a LUMICKS C-Trap equipped with confocal fluorescence microscopy. Some experiments were performed on a laboratory-built instrument equipped with wide-field fluorescence microscopy [39]. Both instruments use comparable, dual optical traps and an identical microfluidic flow cell containing at 
least three parallel laminar-flow channels (Fig. 5.1A, top).

All solutions (beads in $\mathrm{KCl}$-containing phosphate buffer, vimentin in $\mathrm{KCl}$-containing phosphate buffer and pure KCl-containing phosphate buffer) were flushed into the microfluidic chamber using air pressure. Because of laminar flow, they do not mix but flow parallel to each other. For every data set, a fresh pair of beads ( $4.5 \mu \mathrm{m}$ streptavidin coated polystyrene beads, Spherotech Inc.) was captured in the bead channel (channel1) and, by moving the microfluidic chamber with respect to the tweezers, passed through the vimentin channel (channel2) into the buffer channel (channel3), where the measurement was performed after the flow had been stopped. While passing through channel2, vimentin filaments bound to the beads. By moving the second bead closer to the first bead, one filament eventually bound to the second bead. To be sure that there was only one filament between the two beads, confocal imaging was used. Experiments were performed by moving the second bead, while the first stable bead was used for the force measurement.

\subsubsection{Analysis of Optical Tweezers Data}

Data sets were exported in ASCII format from the measuring software (Twom, LUMICKS). The bead diameter was subtracted from the distance measurements, and time and force values were directly used as measured. Only data sets with filaments that were stable for at least three cycles were taken into account. As a threshold to determine the initial filament length, the measured distance at the highest force still smaller than $5 \mathrm{pN}$ at the beginning of the stretching process was used. Using the initial filament length, the strain was calculated for all data sets. The data point density was very high, and missing bead tracking occurred only rarely. Thus, data points with falsely zero distance due to lost tracking were neglected for further analysis. We also neglected data points recorded before the initial filament length was reached and after the filaments broke.

The slopes of repeated force cycles were determined by fitting the force-distance curves for strains between 0 and 0.1 by a straight line. The error bars in Fig. 5.2C show the SEM.

To determine the power-law coefficient $b$, a power-law $\frac{\Delta L}{L_{0}}=A\left(\frac{t}{t_{0}}\right)^{b}$, with the powerlaw prefactor $A$, the initial length $L_{0}$, and the time scale set arbitrarily to $t_{0}=1 \mathrm{~s}$ was 
fitted to the time-dependent change in length $\Delta L$ during the FC experiment.

In order to access the size of the jumps in filament length during FC experiments, we analyzed the distribution of the measured filament lengths. The distribution is shown as histograms on the y-axes in Fig. 5.3 (A and B) and fig. 5.10 (A and B). The plateaus between the jumps correspond to peaks in the filament length distribution. Therefore, the step size can be obtained as the distance between two adjacent peaks. To avoid artifacts due to binning, we used kernel density estimates with a Gaussian kernel with width $\sigma=1.7 \mathrm{~nm}$ (the experimental uncertainty) instead of histograms. Only peaks with a minimal height of $5 \%$ of the highest peak were analyzed.

\subsection{Models}

\subsubsection{Effective Two-State Model}

The filament with length $x$ is modeled as an elastic spring with extension $x_{1}$ coupled to an equivalent freely jointed chain (eFJC) with end-to-end distance $x_{2}$. Since each monomer, and hence the whole filament, is able to change its contour length $L_{C}$ in response to applied forces via the transition of the $\alpha$ helices into an elongated $\beta$ sheet, the contour length, $L_{\mathrm{C}}(F, t)$ becomes a function of time $t$ and force $F(t)$ (for the sake of readability we will state neither explicit nor implicit dependencies going forward).

Assuming a Bell-Evans kinetic for the $\alpha$ - $\beta$ transition for each of the three $\alpha$-helices in the monomer (the three $\alpha$ helices, 1A, 1B, and 2, are indicated by subscripts $i=$ $1,2,3)$, the number $N_{\beta, i}$ of monomers with the $i$ th subunit in the $\beta$ state is described by the following rate equation:

$$
\frac{\mathrm{d} N_{\beta, i}}{\mathrm{~d} t}=\left(N_{0}-N_{\beta, i}\right) \cdot k_{i}^{\alpha \rightarrow \beta} \cdot \exp \left(\frac{F \cdot x_{\alpha, i}}{k_{\mathrm{B}} T}\right)-N_{\beta, i} \cdot k_{i}^{\beta \rightarrow \alpha} \cdot \exp \left(-\frac{F \cdot x_{\beta, i}}{k_{\mathrm{B}} T}\right)
$$

with zero-force reaction rate $k_{i}^{\alpha \rightarrow \beta}$ and potential width $x_{\alpha, i}$ for the $\alpha$ - $\beta$ transition, zero force reaction rate $k_{i}^{\beta \rightarrow \alpha}$ and potential width $x_{\beta, i}$ of the reverse $\beta$ - $\alpha$ transition, and the total number of monomers in a filament $N_{0}=N_{\alpha, i}+N_{\beta, i}$. Here we assume that parallel monomers can be described by a single kinetic, as described by Friddle et al. [40]. From $N_{\beta, i}$ and the lengths of $\alpha$ helices $l_{\alpha, i}, \beta$ sheets $l_{\beta, i}$, and all combined linker regions $l_{0}$, 
we can calculate the contour length $L_{\mathrm{C}}$ of the filament

$$
L_{\mathrm{C}}=N_{0} \cdot l_{0}+\sum_{i}\left(N_{\beta, i} \cdot l_{\beta, i}+N_{\alpha, i} \cdot l_{\alpha, i}\right)
$$

Using the large-force approximation for an eFJC characterized by the Kuhn length $L_{\mathrm{K}}$, we can calculate the extension of the entropic spring $x_{2}$ as

$$
x_{2} \approx L_{\mathrm{C}}\left(1-\frac{F_{\mathrm{K}}}{F}\right)
$$

with the force scale $F_{\mathrm{K}}=\frac{k_{\mathrm{B}} T}{L_{\mathrm{K}}}$. For a semiflexible chain, the Kuhn length is equivalent to twice the persistence length $L_{\mathrm{K}}=2 L_{\mathrm{P}}$ [41]. The serial Hookian spring, which combines elastic contributions from both the filament and the pulling apparatus, is characterized by its spring constant $k_{\text {eff }}$ and its extension $x_{1}$. The force acting on the spring is $F=$ $k_{\mathrm{eff}} \cdot x_{1}=k_{\mathrm{eff}} \cdot\left(x-x_{2}\right)$. With equation (5.3) we obtain:

$$
F=k_{\text {eff }}\left(x-L_{\mathrm{C}}\left(1-\frac{F_{\mathrm{K}}}{F}\right)\right)
$$

When the filament is stretched up to the time $t_{\max }$ and subsequently relaxed with a constant velocity $v$, the extension of the filament changes to

$$
x= \begin{cases}v t & \text { for } t \leq t_{\max } \\ v t_{\max }-v\left(t-t_{\max }\right) & \text { for } t \geq t_{\max }\end{cases}
$$

The refolding of $\alpha$ helices from $\beta$ sheets was neglected previously $\left(k_{i}^{\beta \rightarrow \alpha}=0\right.$ ) because refolding was expected to be suppressed by the high forces during stretching of the filaments but plays an important role during relaxation, which occurs at lower forces. Equation (5.1) includes a nonobvious assumption: We describe all parallel elements by a single effective element, which is possible since unfolding of all parallel elements occurs in a rapid reaction cascade, essentially an all or nothing process (see Fig. 5.2 D). Describing the formation of $\alpha$ helices as the back reaction of the $\alpha$ - $\beta$ transition implies that both reactions have the same specific characteristics, that is, that all parallel elements react rapidly from $\beta$ sheets to $\alpha$ helices. However, we actually know from repeated pulling and relaxation experiments that this assumption is not 
valid, since we observe tensile memory attributed to a fraction of parallel elements remaining in the $\beta$ state (see also Fig.5.2D). Equation (5.1) still provides a useful effective description of both the stretching and the relaxation curve of vimentin filaments, since the partially folded array of parallel elements is similar in length to the all- $\alpha$ configuration. However, $k_{i}^{\beta \rightarrow \alpha}$ and $x_{\beta, i}$ should be strictly considered apparent or effective values and not be over-interpreted numerically. In the effective two-state model, a ULF will still appear as the shorter $\alpha$ helix, even if some of the parallel elements in that ULF are in the extended $\beta$ state. This means that, like in the experimental curves, the energy dissipation in the effective two-state model is an apparent value, since a certain amount of energy is stored as potential energy in the $\beta$ sheets.

Equation (5.1) can be solved numerically for each of the three $\alpha$ helices together with equation (5.2) and (5.4) to obtain $F$. It was shown previously for simple stretching experiments without subsequent relaxation that this simple model is able to reproduce experimental curves over a wide range of loading rates, even if real structural data to determine $l_{\alpha, i}(5.4,13.8$, and $20.9 \mathrm{~nm}), l_{\beta, i}(12.6,32.2$, and $48.7 \mathrm{~nm})$, and $l_{0}(29.7 \mathrm{~nm})$ and literature data for $k_{i}^{\alpha \rightarrow \beta}=3.3 \times 10^{-5} \mathrm{~s}^{-1}$ were used (13). The most likely parameters in the fits of 244 experimental curves were found to be $k_{\text {eff }}=0.23 \mathrm{pN} / \mathrm{nm}, x_{\alpha, 1}=0.11 \mathrm{~nm}$, $x_{\alpha, 2}=0.13 \mathrm{~nm}$, and $x_{\alpha, 3}=0.15 \mathrm{~nm}$. Since the extension curve was only slightly affected by the inclusion of the backwards reaction, we used the parameters determined previously for $k_{\text {eff }}$ and $x_{\alpha, i} \cdot x_{\beta, i}$ was chosen such that there was a fixed ratio between the two potential widths $r_{\mathrm{F}}=\frac{x_{\beta, i}}{x_{\alpha, i}}=2.3$ for all the helices. The back-reaction rate was set to $k_{i}^{\beta \rightarrow \alpha}=5.4 \mathrm{~s}^{-1}$.

To account for creep behavior, we introduce a dashpot with length $x_{3}$ as a third element in series to the entropic and the linear spring such that the total length is $x=x_{1}+x_{2}+x_{3}$. This element shows idealized viscous behavior that can be described by

$$
\frac{\mathrm{d} \epsilon}{\mathrm{d} t}=\frac{\sigma}{\eta}
$$

with the strain $\epsilon$, the viscosity $\eta$ and the stress $\sigma=\frac{F}{A}$ with the area of the cross section $A$. The cross-section area was estimated from the filaments diameter of $d \approx 10 \mathrm{~nm}$. The time derivative of the strain can be related to the time derivative of $x_{3}$ via $\frac{\mathrm{d} x_{3} / L_{0}}{\mathrm{~d} t}=\frac{\mathrm{d} \epsilon}{\mathrm{d} t}$ with a typical value for the initial filament length of $L_{0}=10 \mu \mathrm{m}$. Equation (5.6) is solved 
Chapter 5. Viscoelastic Properties of Vimentin Originate from Nonequilibrium

numerically together with equation (5.1) using $\eta=3.2$ GPas.

The shape of the experimental retraction curve indicates a strong decrease of the persistence length of vimentin filaments during stretching and $\alpha-\beta$ transition. The persistence length of the unstretched filament with all monomers in $\alpha$-helical configuration is known from literature to be $L_{\mathrm{P}, \alpha}=1000 \mathrm{~nm}$, leading to the force scale $F_{\mathrm{K}, \alpha} \approx 0.002 \mathrm{pN}$ [42]. However, the shape of the relaxation curve suggested a substantially smaller persistence length (see fig. 5.5). Therefore, we introduced a second persistence length for the stretched filament $L_{\mathrm{P}, \beta}$ with a corresponding force scale $F_{\mathrm{K}, \beta}$. We interpolated linearly between the two different force scales, $F_{\mathrm{K} \alpha}$ for $N_{\beta, i}=0$ and $F_{\mathrm{K} \beta}$ for $N_{\beta, i}=N_{0}$, leading to a strain dependence of the persistence length.

\subsubsection{Analytical Solution for FC Conditions}

Under FC conditions, that is, at constant force, the analytical solution of equation (5.1) becomes straightforward. Since we rarely observe any shortening of the filament during FC experiments, even at the lowest force of $50 \mathrm{pN}$, we can neglect the restoration of $\alpha$ helices from $\beta$ sheets. When the force $F$ is constant, the force-dependent reaction rate $k_{i, F}^{\alpha \rightarrow \beta}=k_{i, 0}^{\beta \rightarrow \alpha} \cdot \exp \left(\frac{F \cdot x_{\alpha, i}}{k_{\mathrm{B}} T}\right)$ also becomes a constant. Therefore, the solution of equation (5.1) reads

$$
N_{\alpha, i, F}=N_{0} \cdot \exp \left(-k_{i, F}^{\alpha \rightarrow \beta} \cdot t\right)
$$

Equation (5.2) is then used to calculate $L_{C}$, and equation (5.3) allows us to calculate $x_{2} . x=x_{1}+x_{2}$ is used to calculate the filament length. The strain is calculated using $\epsilon=\frac{x-L_{0}}{L_{0}}$ with a typical value for the initial filament length $L_{0}=10 \mu \mathrm{m}$. The value used for $L_{0}$ only affects the contribution of the elastic extension $x_{1}$ to the strain $\epsilon$ and has therefore only a minor impact on the resulting curves.

\subsubsection{Simulation of Unfolding and Refolding of Parallel Elements}

More complex behavior of vimentin IFs, such as the tensile memory, cannot be described without explicitly considering the architecture of ULFs consisting of parallel elements, as sketched in fig. 5.6. It is convenient to introduce dimensionless force $\phi=F / F_{\alpha}$ by normalization with the force scale of the $\alpha$ - $\beta$ transition $F_{\alpha}$, dimensionless length scale $\lambda=L / \Delta L$ using the length difference $\Delta L$ between $\alpha$ state and $\beta$ state 
and dimensionless time $\tau=t \cdot k_{0}^{\alpha \rightarrow \beta}$ with the zero-force reaction rate $k_{0}^{\alpha \rightarrow \beta}$ of the $\alpha$ - $\beta$ transition of a single monomer.

The dimensionless stiffness of the filament $\kappa_{\mathrm{TOT}}=\frac{\Delta \phi}{\Delta \lambda}$ depends on the stiffness of the ULF $\kappa_{U L F}$ and the number of ULFs in the filament $N_{\mathrm{S}}$

$$
\kappa_{\mathrm{TOT}}=\left(\sum_{j=1}^{N_{\mathrm{S}}} \frac{1}{\kappa_{\mathrm{ULF}, j}}\right)^{-1}
$$

The simplest model to describe the elasticity of the ULF is a parallel arrangement of $N_{\mathrm{T}}$ springs with stiffness $\kappa_{\mathrm{P}}$. Each of these springs is arranged in series to an element that can change its length in a force dependent manner, representing the $\alpha$ helices that can switch into an extended $\beta$ sheet, with $N_{\mathrm{P}}$ elements in $\alpha$-helical configuration. Together with an additional spring with stiffness $\kappa_{V}$ serial to this element representing linkers and the connection between ULFs, the stiffness of one ULF is:

$$
\kappa_{\mathrm{ULF}, j}=\left(\frac{1}{\kappa_{\mathrm{V}}}+\frac{1}{\kappa_{\mathrm{P}} \cdot N_{\mathrm{P}, j}}\right)^{-1}
$$

To illustrate that this mechanism is suitable to generate tensile memory, we performed MC simulations. For simplicity, we neglected entropic contributions to the elasticity and viscous contributions and used only one extendable element for each ULF as opposed to the three helices used in the numerical description above.

Each ULF $j$ is characterized by the number of elements $N_{\mathrm{P}, j}$ in the shorter $\alpha$-helical state. Since the elements can switch between $\alpha$ state and $\beta$ state, the number of $\alpha$ helical elements in each individual ULF is described by the master equation

$$
\frac{\mathrm{d} P_{N}}{\mathrm{~d} t}=r_{N-1}^{\beta \rightarrow \alpha} \cdot P_{N-1}+r_{N+1}^{\alpha \rightarrow \beta} \cdot P_{N+1}-\left[r_{N}^{\beta \rightarrow \alpha}+r_{N}^{\alpha \rightarrow \beta}\right] \cdot P_{N}
$$

where $P_{N}$ represents the probability that $N$ parallel elements are in the $\alpha$-helical state, $r_{N}^{\alpha \rightarrow \beta}$ is the rate of the $\alpha-\beta$ transition and $r_{N}^{\beta \rightarrow \alpha}$ is the rate of the reverse reaction.

When all parallel elements are in the elongated state, that is, the $\beta$-state, the ULF is extended by $\Delta L$. Since the length scale is normalized by $\Delta L$, the dimensionless exten- 
sion of the $j$ th ULF $\lambda_{j}$ can be described as

$$
\lambda_{j}= \begin{cases}0 & \text { for } N_{\mathrm{P}, j}>0 \\ 1 & \text { for } N_{\mathrm{P}, j}=0\end{cases}
$$

The total extension of the filament is $\lambda_{\mathrm{TOT}}=\sum_{j=1}^{N_{\mathrm{S}}} \lambda_{j}$. The total force acting on a filament with end-to-end distance $x$ is given by $\phi=\kappa_{\mathrm{TOT}} \cdot\left(x-\lambda_{\mathrm{TOT}}\right)$. The time-dependent end-to-end distance of the filament $x(t)$ is calculated according to

$$
x= \begin{cases}v \cdot \tau & \text { for } \tau \leq \tau_{\max } \\ x_{\max }-v\left(\tau-\tau_{\max }\right) & \text { for } \tau \geq \tau_{\max }\end{cases}
$$

using the dimensionless velocity $v=\delta \lambda / \delta \tau$ and the maximal extension $x_{\max }=v \cdot \tau_{\max }$ at the time point $\tau_{\max }$. The reaction rates were chosen similar to the ones for the effective two-state model described above. The rates are a function of the force acting on each of the parallel elements $\phi_{\mathrm{P}, j}$. If there is at least one element in $\alpha$ state, then each of these elements experiences $\phi_{\mathrm{P}, j}=\frac{\phi}{N_{\mathrm{P}, j}}$, while the extended elements are force-free. The reaction rate $r_{N_{\mathrm{P}, j}}^{\alpha \rightarrow \beta}$ can therefore be expressed as

$$
r_{N_{\mathrm{P}, j}}^{\alpha \rightarrow \beta}=N_{\mathrm{P}, j} \cdot \exp \left(\frac{\phi}{N_{\mathrm{P}, j}}\right)
$$

If all elements of an ULF are in the extended $\beta$ sheet conformation, then the force will be shared between them such that $\phi_{\mathrm{P}, j}=\frac{\phi}{N_{\mathrm{T}}}$. Therefore, the reaction rate is

$$
r_{N_{\mathrm{P}, j}}^{\beta \rightarrow \alpha}= \begin{cases}\left(N_{\mathrm{T}}-N_{\mathrm{P}, j}\right) \cdot \gamma & \text { for } N_{\mathrm{P}, j}>0 \\ N_{\mathrm{T}} \cdot \gamma \cdot \exp \left(\frac{-\phi}{N_{\mathrm{T}}}\right) & \text { for } N_{\mathrm{P}, j}=0\end{cases}
$$

using the normalized rebinding constant $\gamma=\frac{k_{0}^{\beta \rightarrow \alpha}}{k_{0}^{\alpha \rightarrow \beta}}$ with the zero-force reaction rate $k_{0}^{\beta \rightarrow \alpha}$ of refolding. The free-energy difference between $\alpha$ helix and $\beta$ sheet is $\frac{\Delta G}{k_{\mathrm{B}} T}=$ $\ln (\gamma)$.

This set of equations was solved by MC methods using the Gillespie algorithm with initial condition $N_{\mathrm{P}}(t=0)=N_{\mathrm{T}}\left(1-\exp \left(\frac{-\Delta G}{k_{\mathrm{B}} T}\right)\right)$, that is, the system being in thermal 
equilibrium. When multiple consecutive pulling cycles are simulated, $N_{\mathrm{P}, j}$ at the end of each cycle was used as the initial condition for the next cycle. The parameters used to produce Fig. 5.2 (B and D) were $N_{\mathrm{S}}=100, N_{\mathrm{T}}=32, \kappa_{\mathrm{P}}=10, \kappa_{\mathrm{V}}=100, v=2000$ and $\frac{\Delta G}{k_{\mathrm{B}} T}=2$. The Jupyter Notebook $[43,44]$ used to produce the data shown in Fig. 5.2 (B and D) is included as supplementary information. 
Chapter 5. Viscoelastic Properties of Vimentin Originate from Nonequilibrium

Acknowledgements: We are thankful for the discussions and technical support by S. Bauch, A. Schepers, J. Kraxner, F. Savič, and B. Geil.

Funding: This project has received funding from the European Research Council under the European Union's Horizon 2020 research and innovation programme (Consolidator grant agreement no. 724932 and Laserlab-Europe grant agreement $\mathrm{n}^{\circ}$ 654148). The work was further financially supported by the Deutsche Forschungsgemeinschaft in the framework of SFB755 (project B7) and SFB937 (project A17) as well as the Göttingen Graduate School for Neurosciences, Biophysics, and Molecular Biosciences (Grant No. GSC 226/2).

Author contributions: The project was conceived by S.K. J.B. performed all experiments with assistance from A.C. and J.C.D.; the experiments were partly performed in the laboratories of, and in collaboration with, E.J.G.P and G.J.L.W. H.W. developed and implemented the models and performed all simulations; J.B. and H.W. analyzed the data; S.K. and A.J. supervised the project; J.B., H.W., A.J. and S.K. wrote the manuscript. All authors read and approved the manuscript.

Competing interests: The authors declare no conflict of interests.

Data and materials availability: Correspondence and requests for materials should be addressed to S.K. (sarah.koester@phys.uni-goettingen.de) or A.J. (andreas.janshoff@chemie.uni-goettingen.de). 


\subsection{Supplementary Materials}

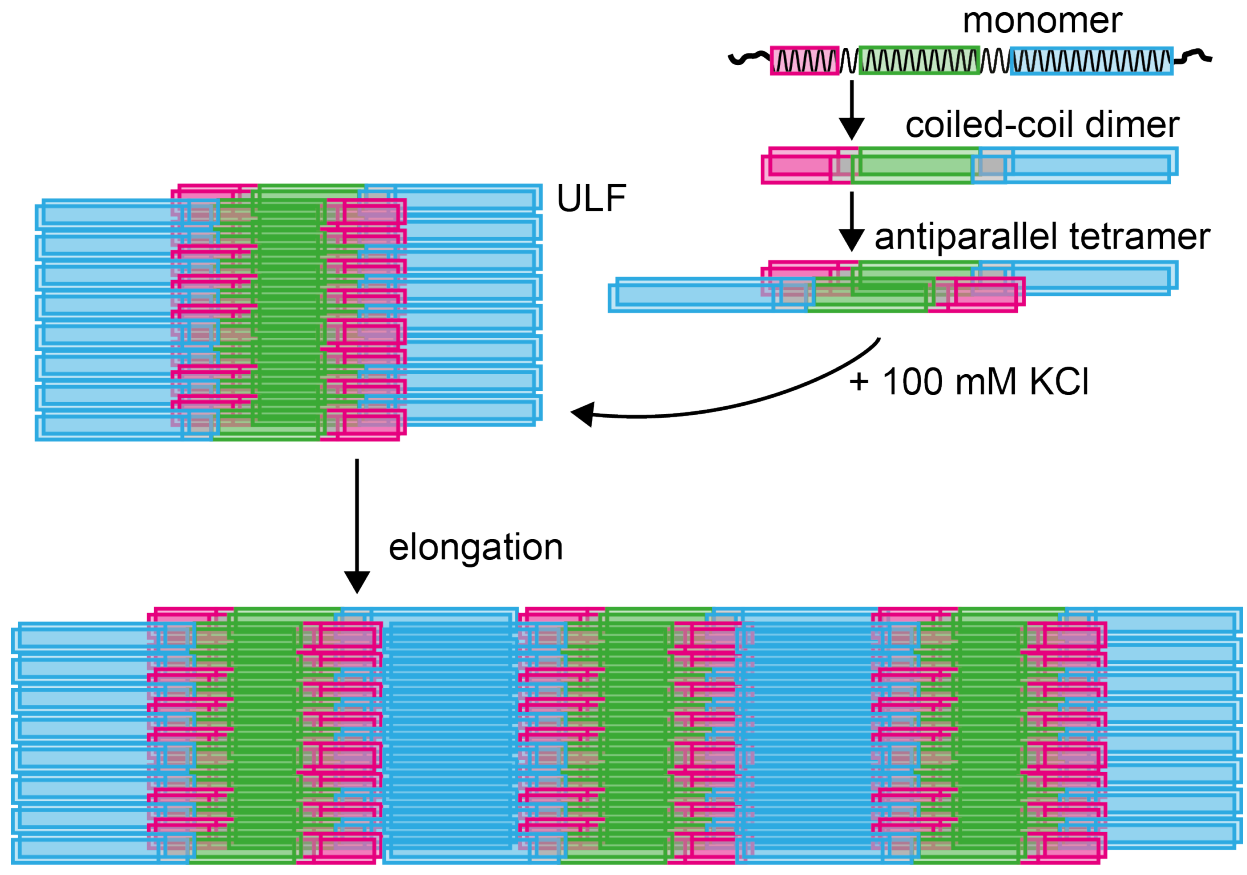

Figure 5.4: Vimentin Assembly: IF formation follows a complex and hierarchical scheme.

Vimentin Assembly: IF formation follows a complex and hierarchical scheme. Lateral assembly of monomers via parallel coiled-coil dimers yields half-staggered anti-parallel tetramers. Upon the addition of salt (in this case $100 \mathrm{mM} \mathrm{KCl)} \mathrm{unit-length} \mathrm{filaments} \mathrm{(ULFs)} \mathrm{are} \mathrm{formed.} \mathrm{Longitudinal}$ annealing of these ULFs results in mature, elongated filaments of $10 \mathrm{~nm}$ in diameter. This hierarchical architecture, together with the secondary structure of each monomer, provides a basis for understanding the structure-function relationship in the context of filament mechanics. 


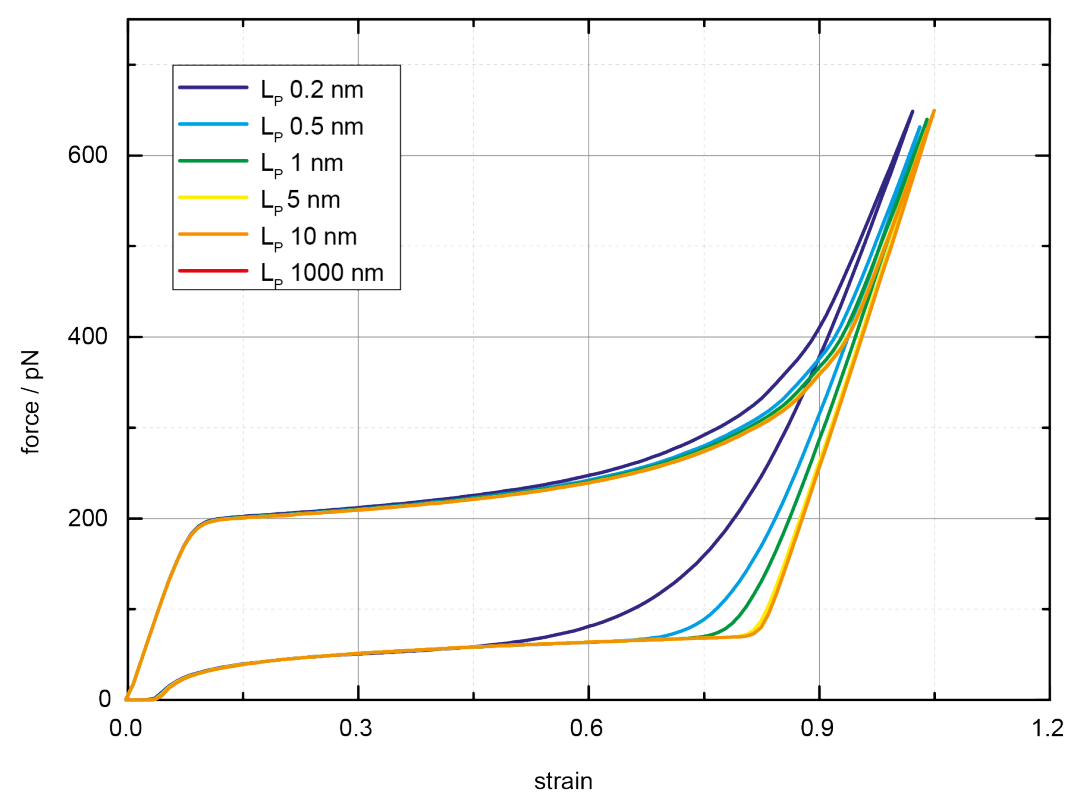

Figure 5.5: Comparison of calculated (two-state-model) stretching and relaxation curves for different values of the persistence lengths $L_{P}$ (color-coded; see legend). Persistence lengths of $0.5 \mathrm{~nm}$ and $1 \mathrm{~nm}$ are able to reproduce the convex shape observed experimentally. $L_{P}$ values for relaxed vimentin were reported to be on the order of a few $\mu \mathrm{m}$ [42, 45]. LP of a rod scales with the filament radius as $r^{4}$. Assuming volume incompressibility, thinning of the filament at $120 \%$ strain only predicts a reduction of $L_{P}$ by a factor of approximately 5 , which is not sufficient to reproduce the shape of the retraction curve, i.e. explain the low $L_{P}$. A more dramatic decrease in $L_{P}$ could, however, occur if the interaction between parallel elements was partially lost due to the $\alpha$ - $\beta$-transition, such that they do not act as a single chain, but behave like multiple uncoupled, parallel worm-like chains. 


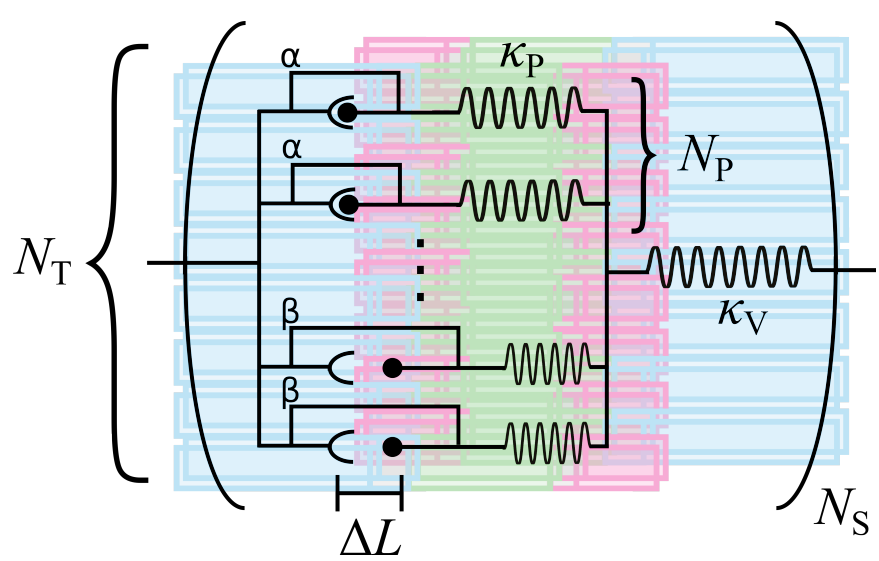

Figure 5.6: Sketch of the equivalent circuit diagram used for setting up the MC simulations.
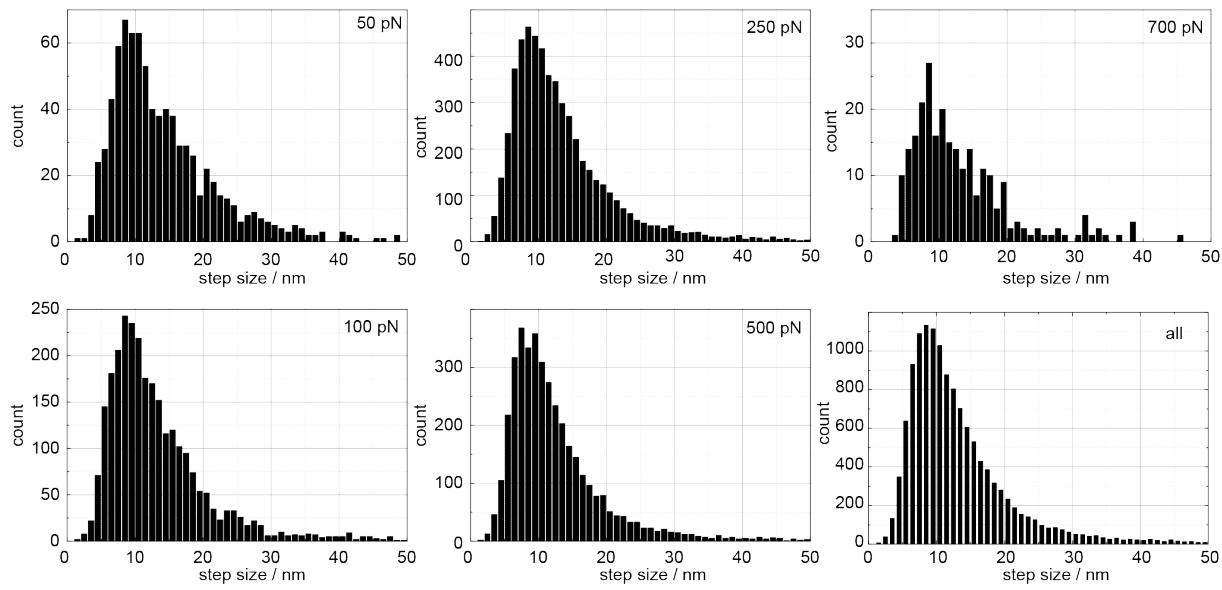

Figure 5.7: Step size analysis of force clamp data sets. Histograms show the step size distribution per force $(50,100,250,500$ and $700 \mathrm{pN})$ and the total distribution of step sizes. 

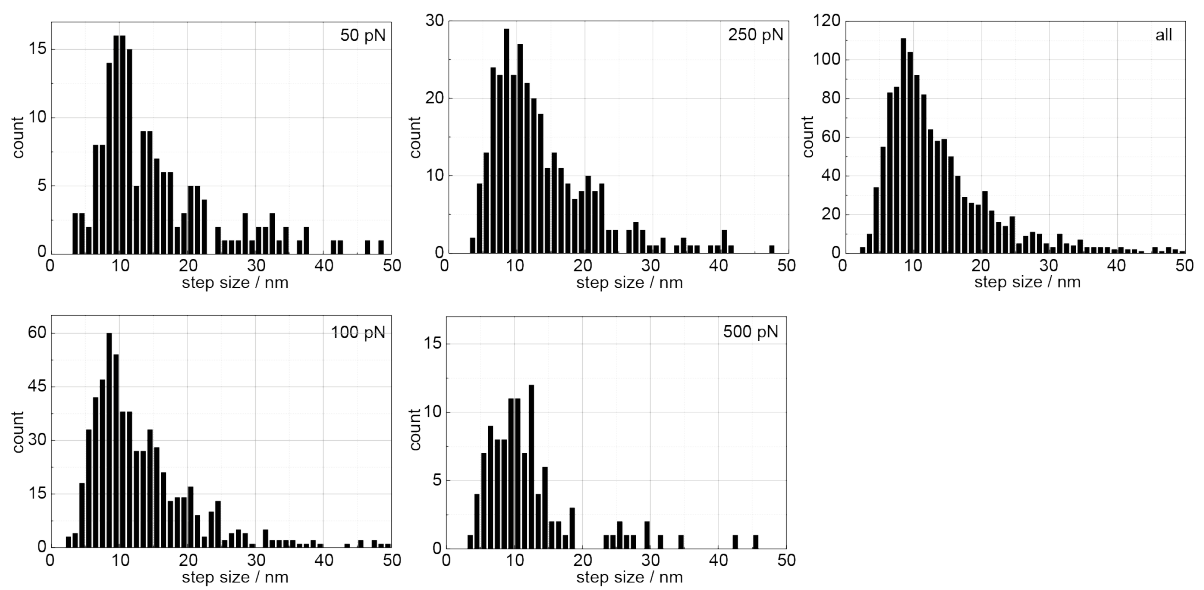

Figure 5.8: Step size analysis of data sets with filaments covalently bond to beads via malemide-chemistry. Histograms show the step size distribution per force clamp force (50,100, 250 and $500 \mathrm{pN})$ and the total distribution of step sizes. Since the step size distribution is comparable to filaments coupled via biotin-streptavidin interactions, we can exclude the filament-bead bond as the cause for the steps. The steps can therefore unequivocally be assigned to intrinsic structural changes in the filament.
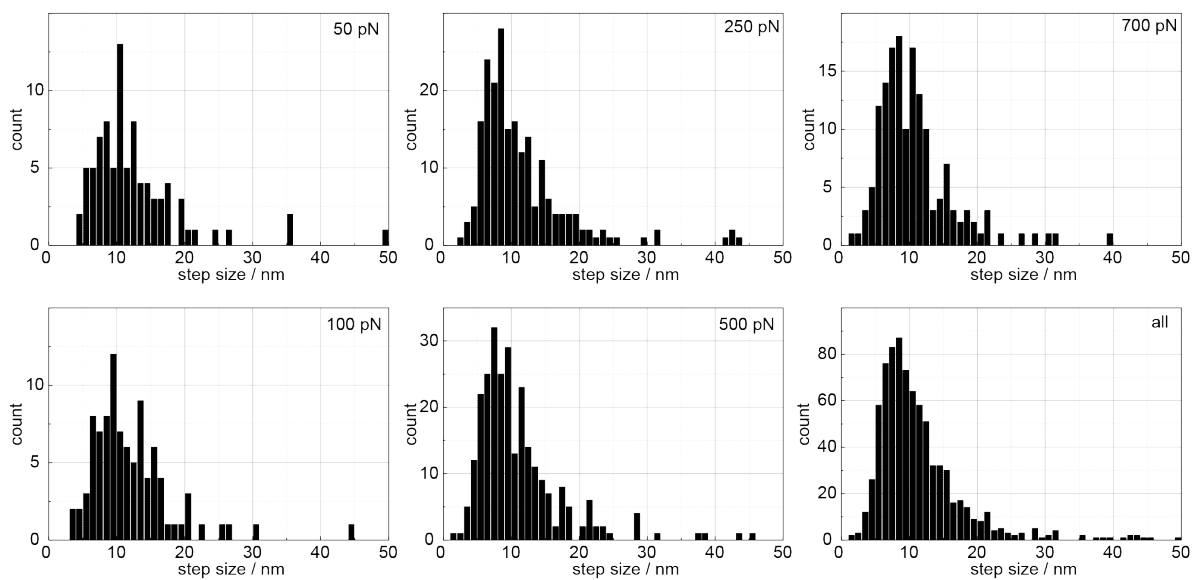

Figure 5.9: Step size analysis of relaxation data sets. Histograms show the step size distribution per starting force $(50,100,250,500$ and $700 \mathrm{pN})$ and the total distribution of step sizes. Individual filaments were pulled until a certain force was reached, and were allowed to relax afterwards without force feedback. 
A
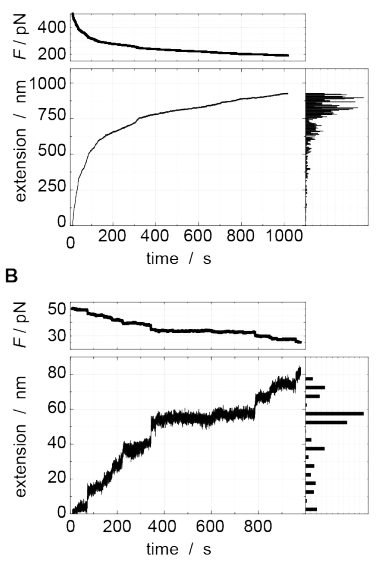

C

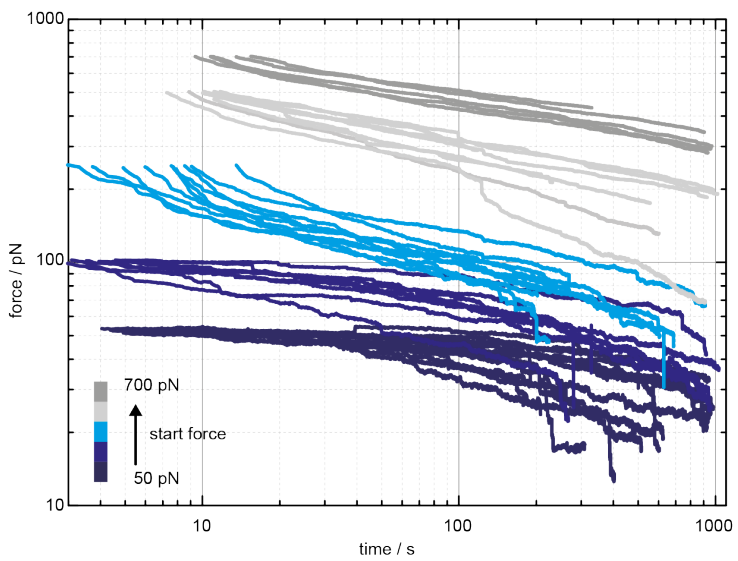

Figure 5.10: Vimentin relaxation experiments starting from different forces. $(A, B)$ Examples of single vimentin filaments. Filaments were stretched until a force of 500 or $50 \mathrm{pN}$, respectively, was reached and were allowed to relax while the optical tweezers were kept at a constant position. The upper graph in each example shows the force vs. time, the lower one shows the filament extension $v$. time. On the right, the filament length is shown as a histogram to illustrate steps in the extension curve more clearly. (C) Log-log plot of force vs. time for creep experiments on numerous vimentin filaments at starting forces between 50 and $700 \mathrm{pN}$ (see color code). The time offset of several seconds is due to the experimental setup. Filaments are stretched to the starting force while the data set is already acquired. Data recorded before the starting force is reached, are not shown in this plot. 


\subsubsection{Movie S1}

The supplementary movie shows the epi-fluorescence recording of the stretching cycle which is shown in Figure 5.2A in the main text. This video clearly shows that the filament softening does not result in buckling events when the filament is relaxed, but the filament stays straight and fully in focus. The buckling events, which are visible in the video, are due to the fact that the beads were moved closer together than the initial filament length at the end of most of the stretching-relaxation cycles. To clarify this point, the raw data of this measurement are shown in fig. 5.11. Data acquisition was started with a delay relating to the fluorescence video. The beads are moved close to each other in the beginning of the video to ensure that the filament is completely relaxed, then the force measured by the system is set to zero, data acquisition is started and the cycle begins. This means that the buckling in the very beginning of the video is not visible in the data set. The length of the filament (the distance between the two beads at the last force value below $5 \mathrm{pN}$, as defined in the Material and Methods section: "Analysis of optical tweezers data") measured in this experiment was $8.996 \mu \mathrm{m}$. This value is marked by a blue line in all graphs in fig. 5.11.

Neither with epi-fluorescence nor with confocal fluorescence microscopy it is possible to resolve buckling of individual monomers within one ULF. However, it is true that length differences of the monomers as they occur when some of the monomers within the same ULF return to the $\alpha$ state while others are still in the $\beta$ state (illustrated in the cartoon in fig. 5.6) have to result in buckling or compression of the "longer" monomers. Note, however, that a monomer can even be partly in $\alpha$ state and partly in $\beta$ state. 


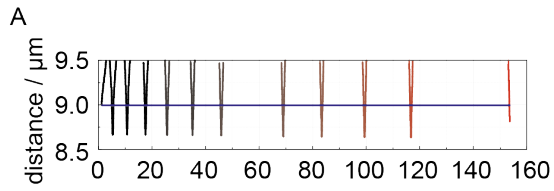

B
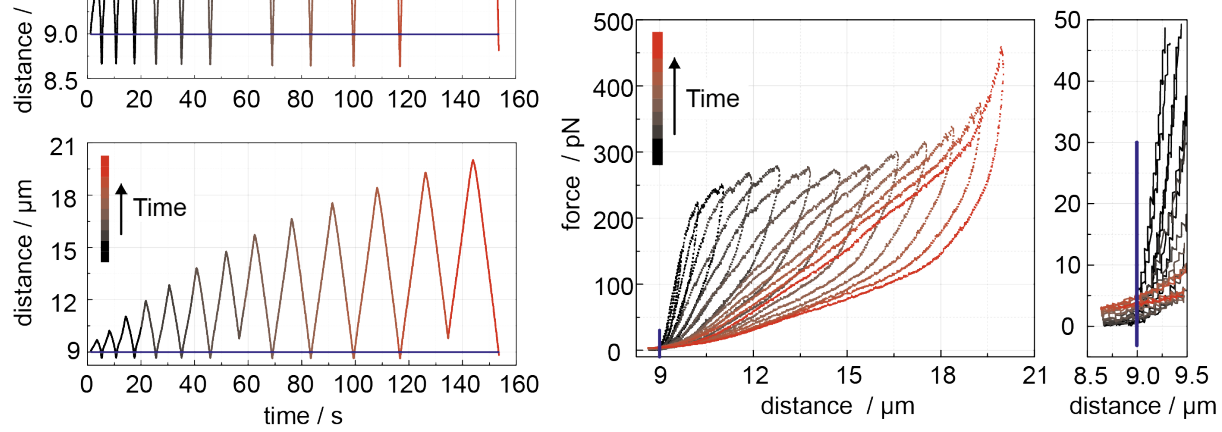

Figure 5.11: Data corresponding to the cycle shown in movie S1. The blue line in all graphs marks the filament length. (A) Bottom: distance vs. time, full data set, also shown in fig. 5.2A top; Top: distance vs. time, zoom in $y$-direction to focus on the fact that the two beads are moved closer together than the initial filament length. (B) Left: force $v$ s. distance, full data set, also shown in fig. 5.2A bottom; Right: force $v$ s. distance, zoom in $x$ and $y$-direction.

A

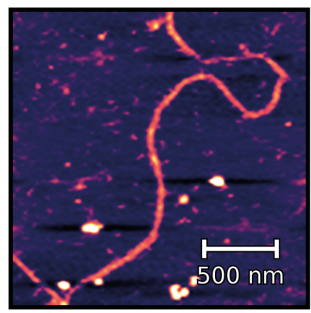

B

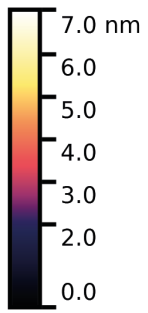

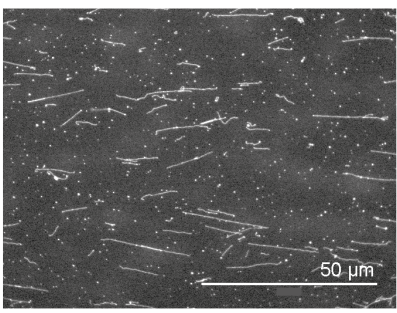

C

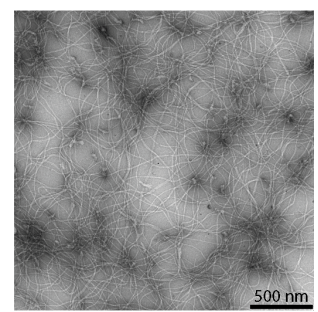

Figure 5.12: Quality control of labeled, assembled vimentin filaments. All three images show ATTO647Nand biotin-labeled vimentin filaments with a total labeling ratio of less than $10 \%$ (A) atomic force microscopy (AFM) (image from Ref. [14]), (B) epi-fluorescence microscopy, (C) electron microscopy (EM). 
Chapter 5. Viscoelastic Properties of Vimentin Originate from Nonequilibrium

Conformational Changes

A

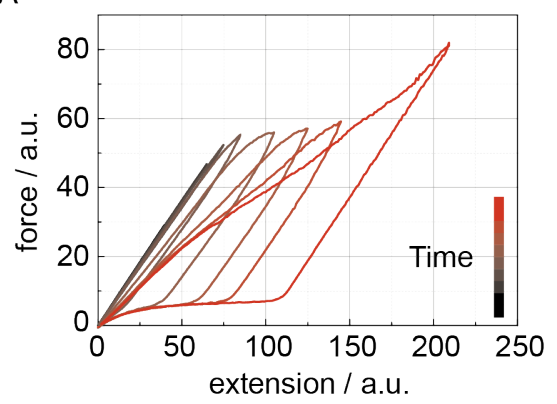

B

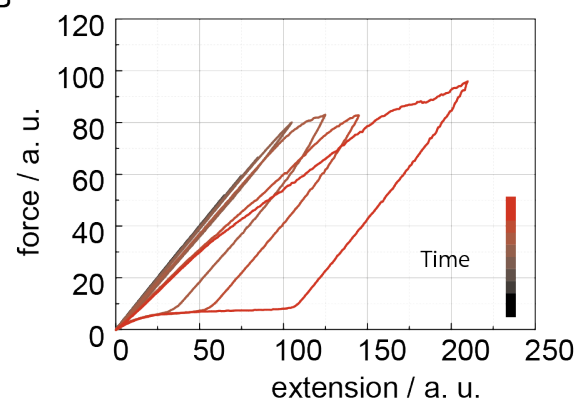

Figure 5.13: Simulations, cycles, and comparison for different numbers of monomers per ULF. (A) Kinetic $M C$ simulations as shown in Fig. 5.2B of the main text, 32 monomers per ULF/cross-section of the filament. (B) Corresponding simulation for 48 monomers per ULF/cross-section. No qualitative difference is observed. 


\section{References}

1. Szeverenyi, I. et al. The Human Intermediate Filament Database: comprehensive information on a gene family involved in many human diseases. Hum. Mutat. 29, 351-360 (2008).

2. Seltmann, K., Fritsch, A. W., Käs, J. A. \& Magin, T. M. Keratins Significantly Contribute to Cell Stiffness and Impact Invasive Behavior. P. Natl. Acad. Sci. USA 110, 18507-18512 (2013).

3. Mendez, M. G., Restle, D. \& Janmey, P. A. Vimentin Enhances Cell Elastic Behavior and Protects Against Compressive Stress. Biophys J 107, 314-323 (2014).

4. Ramms, L. et al. Keratins as the Main Component for the Mechanical Integrity of Keratinocytes. P. Natl. Acad. Sci. USA 110, 18513-18518 (2013).

5. Fuchs, E. \& Cleveland, D. W. A Structural Scaffolding of Intermediate Filaments in Health and Disease. Science 279, 514-519 (1998).

6. Herrmann, H. et al. Structure and Assembly Properties of the Intermediate Filament Protein Vimentin: The Role of Its Head, Rod and Tail Domains. J. Mol. Biol. 264, 933-953 (1996).

7. Chernyatina, A. A., Guzenko, D. \& Strelkov, S. V. Intermediate Filament Structure: the Bottom-Up Approach. Curr. Opin. Cell Biol. 32C, 65-72 (2015).

8. Aebi, U. The fibrillar substructure of keratin filaments unraveled. 97, 1131-1143 (1983).

9. Bendit, E. G. A Quantitative X-Ray Diffraction Study of the Alpha-Beta Transformation in Wool Keratin. Text. Res. J. 30, 547-555 (1960).

10. Burte, H. \& Halsey, G. A New Theory of Non-Linear Viscous Elasticity. Text. Res. J. 17, 465-476 (1947).

11. Köster, S., Weitz, D. A., Goldman, R. D., Aebi, U. \& Herrmann, H. Intermediate Filament Mechanics in Vitro and in the Cell: From Coiled Coils to Filaments, Fibers and Networks. Curr. Opin. Cell Biol. 32C, 82-91 (2015).

12. Kreplak, L. \& Fudge, D. Biomechanical Properties of Intermediate Filaments: from Tissues to Single Filaments and Back. Bioessays 29, 26-35 (2007). 
Chapter 5. Viscoelastic Properties of Vimentin Originate from Nonequilibrium

13. Block, J., Schroeder, V., Pawelzyk, P., Willenbacher, N. \& Koster, S. Physical Properties of Cytoplasmic Intermediate Filaments. Biochim. Biophys. Acta 1853, 30533064 (2015).

14. Block, J. et al. Nonlinear Loading-Rate-Dependent Force Response of Individual Vimentin Intermediate Filaments to Applied Strain. Phys. Rev. Lett. 118, 048101 (2017).

15. Kreplak, L., Herrmann, H. \& Aebi, U. Tensile Properties of Single Desmin Intermediate Filaments. Biophys J 94, 2790-2799 (2008).

16. Kreplak, L., Bär, H., Leterrier, J. F., Herrmann, H. \& Aebi, U. Exploring the Mechanical Behavior of Single Intermediate Filaments. J. Mol. Biol. 354, 569-577 (2005).

17. Qin, Z., Kreplak, L. \& Buehler, M. J. Hierarchical Structure Controls Nanomechanical Properties of Vimentin Intermediate Filaments. PLOS One 4, e7294 (2009).

18. Rief, M. Elastically Coupled Two-Level Systems as a Model for Biopolymer Extensibility. Phys. Rev. Lett. 81, 4764-4767 (1998).

19. Minin, K. A., Zhmurov, A., Marx, K. A., Purohit, P. K. \& Barsegov, V. Dynamic Transition From Alpha-Helices to Beta-Sheets in Polypeptide Coiled-Coil Motifs. J. Am. Chem. Soc. 139, 16168-16177 (2017).

20. Liu, W., Carlisle, C. R., Sparks, E. A. \& Guthold, M. The Mechanical Properties of Single Fibrin Fibers. J. Thromb. Haemost. 8, 1030-1036 (2010).

21. Gosline, J. M., Guerette, P. A., Ortlepp, C. S. \& Savage, K. N. The Mechanical Design of Spider Silks. From fibroin sequence to mechanical function. J. Exp. Biol. 202, 3295-3303 (1999).

22. Brown, A. E. X., Litvinov, R. I., Discher, D. E., Purohit, P. K. \& Weisel, J. W. Multiscale Mechanics of Fibrin Polymer. Gel Stretching With Protein Unfolding and Loss of Water. Science 325, 741-744 (2009).

23. Kellermayer, M. S. Folding-Unfolding Transitions in Single Titin Molecules Characterized with Laser Tweezers. Science 276, 1112-1116 (1997).

24. Rief, M., Gautel, M., Oesterhelt, F., Fernandez, J. M. \& Gaub, H. E. Reversible Unfolding of Individual Titin Immunoglobulin Domains by AFM. Science 276, 11091112 (1997). 
25. Münster, S. et al. Strain History Dependence of the Nonlinear Stress Response of Fibrin and Collagen Networks. P. Natl. Acad. Sci. USA 110, 12197-12202 (2013).

26. Nicolet, S., Herrmann, H., Aebi, U. \& Strelkov, S. V. Atomic Structure of Vimentin Coil 2. J. Struct. Biol. 170, 369-376 (2010).

27. Kollmannsberger, P. \& Fabry, B. Linear and Nonlinear Rheology of Living Cells. Annu. Rev. Mater. Res. 41, 75-97 (2011).

28. Desprat, N., Richert, A., Simeon, J. \& Asnacios, A. Creep Function of a Single Living Cell. Biophys J 88, 2224-2233 (2005).

29. Lin, Y.-C. et al. Origins of Elasticity in Intermediate Filament Networks. Phys. Rev. Lett. 104 (2010).

30. Lin, Y.-C. et al. Divalent Cations Crosslink Vimentin Intermediate Filament Tail Domains to Regulate Network Mechanics. J. Mol. Biol. 399, 637-644 (2010).

31. Schopferer, M. et al. Desmin and Vimentin Intermediate Filament Networks: Their Viscoelastic Properties Investigated by Mechanical Rheometry. J. Mol. Biol. 388, 133-143 (2009).

32. Fudge, D. et al. The Intermediate Filament Network in Cultured Human Keratinocytes is Remarkably Extensible and Resilient. PLOS One 3, e2327 (2008).

33. Pinto, N. et al. Self-Assembly Enhances the Strength of Fibers Made from Vimentin Intermediate Filament Proteins. Biomacromolecules 15, 574-581 (2014).

34. Mártonfalvi, Z. et al. Low-Force Transitions in Single Titin Molecules Reflect a Memory of Contractile History. J. Cell Sci. 127, 858-870 (2014).

35. Schnittler, H.-J., Schmandra, T. \& Drenckhahn, D. Correlation of Endothelial Vimentin Content with Hemodynamic Parameters. Histochem. Cell Biol. 110, 161167 (1998).

36. Herrmann, H. \& Aebi, U. Intermediate Filaments: Molecular Structure, Assembly Mechanism, and Integration into Functionally Distinct Intracellular Scaffolds. Annu. Rev. Biochem. 73, 749-789 (2004).

37. Winheim, S. et al. Deconstructing the Late Phase of Vimentin Assembly by Total Internal Reflection Fluorescence Microscopy (TIRFM). PLOS One 6, e19202 (2011). 
Chapter 5. Viscoelastic Properties of Vimentin Originate from Nonequilibrium

38. Janissen, R. et al. Invincible DNA Tethers: Covalent DNA Anchoring for Enhanced Temporal and Force Stability in Magnetic Tweezers Experiments. Nucleic Acids Res. 42, e137 (2014).

39. Brouwer, I. et al. Sliding Sleeves of XRCC4-XLF Bridge DNA and Connect Fragments of Broken DNA. Nature 535, 566-569 (2016).

40. Friddle, R. W., Noy, A. \& de Yoreo, J. J. Interpreting the Widespread Nonlinear Force Spectra of Intermolecular Bonds. P. Natl. Acad. Sci. USA 109, 13573-13578 (2012).

41. Flory, P. J. Statistical Mechanics of Chain Molecules (Interscience Publ, New York, 1969).

42. Nöding, B. \& Köster, S. Intermediate Filaments in Small Configuration Spaces. Phys. Rev. Lett. 108, 088101 (2012).

43. Oliphant, T. E. Python for Scientific Computing. Comput. Sci. Eng. 9, 10-20 (2007).

44. Perez, F. \& Granger, B. E. IPython. A System for Interactive Scientific Computing. Comput. Sci. Eng. 9, 21-29 (2007).

45. Mücke, N. et al. Assessing the Flexibility of Intermediate Filaments by Atomic Force Microscopy. J. Mol. Biol. 335, 1241-1250 (2004). 


\section{Chapter 6}

\section{Mechanical Response of \\ Glutaraldehyde Stabilized}

Vimentin IFs

\subsection{Introduction}

Glutaraldehyde has been widely used to stabilize and immobilize proteins, e.g. in histochemistry, AFM or EM [1, 2]. A standard protocol to prepare EM samples of Class III IFs involves glutaraldehyde fixation prior to the adsorption to the EM grid in order to preserve their architecture [3]. Depending on the conditions glutaraldehyde is known to react with about half of the amino acids in a reversible manner [1]. Yet, the highest reactivity is reported for lysine and the lowest reversibility was found for $\mathrm{pH} 7.0$ to 9.0 [1].

AFM images of glutaraldehyde fixed vimentin IFs on a mica surface revealed a pattern of the surface structure with a repetition length of $21 \mathrm{~nm}$ along the axis of the filament both for measurements in air and under physiological buffer conditions [4]. However, Mücke et al. found a correlation between the morphology of glutaraldehyde fixed vimentin and the surface type it is adsorbed to indicating that the observed structures 
were affected by the underlying substrate. Depending on the surface type the filaments did more or less flatten and spontaneously rearrange without disrupting the global filament structure [5]. AFM measurements of glutaraldehyde stabilized single vimentin filaments spanned over holes on a structured substrate (Fig. 2.2 B) showed a two to three times increase in the bending modulus compared to non-stabilized filaments [2].

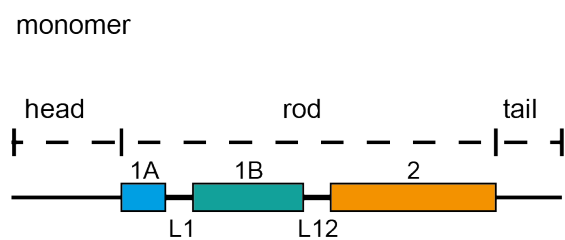

dimer

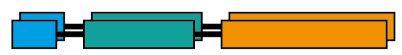

tetramer types

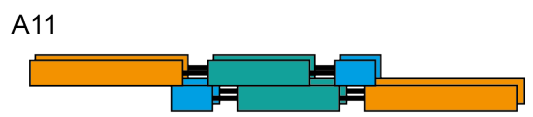

A22

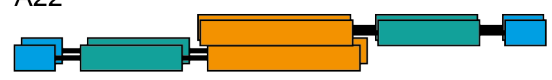

A12

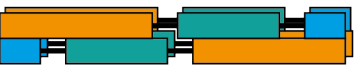

Figure 6.1: General monomer and dimer structure and different types of tetramers of vimentin IFs. The A11 tetramer is most likely the basis for further lateral and subsequently longitudinal assembly. The A22 dimer was observed in vitro [6] and is also found as part of the filament as the overlapping part of two ULFs has the form of several A22 tetramers. The A12 "tetramer" does not exist in the tertameric form, but was only found as cross-linking product in polymers and mature filaments.

In the early $1990^{\text {th }}$ Steinert et al. looked into the lysine-lysine cross-linking by disulfosuccinimidyl tartrate (DST) in vimentin filaments [6]. They found 16 cross-links, where five were located in the parallel coiled coil dimer, and eleven between different dimers. Fig. 6.1 shows a sketch of the vimentin monomer, dimer and the different tetramer types. Nine of the eleven cross-links were found in half staggered tetramers - six in the A11 and three in the A22 tetramer. The A11 tetramer is believed to be the subunit of ULFs [7], but when two ULFs come together, the overlapping parts combine and form approximately an A22 tetramer. The last two cross-link types were only found in mature filaments, and were located in fully overlapping dimers in an A12 arrangement [6]. Downing reanalyzed the data obtained by Steinert et al. and pointed out that cross-links are unlikely to occur between two lysines which are both within an $\alpha$-helical region [8]. In fact, the five cross-links in the dimer are between lysines out- 
side of $\alpha$-helices and only one of the reported cross-links connects two lysines which are both within an $\alpha$-helix [8]. From the amino acid sequence and structure of the A12 tetramer there would be at least eight more pairs of lysine that could be candidates for cross-linking but did not appear [8]. However, this might be due to the architecture of the mature filament as this tetramer is not found as an assembly intermediate.

Taking these studies together, there is evidence that inter-dimer sliding $[9,10]$ would not be easily possible anymore in the cross-linked filament. Unfolding of $\alpha$-helices (e.g. [10-12]) instead might still be possible, however, it would most likely be necessary that several parallel helices do unfold at the same time.

Here the mechanical properties of single, glutaraldehyde stabilized vimentin IFs are directly tested by OT measurements and the results are compared to the properties of non-stabilized filaments which are presented in the two previous chapters. Compared to the described AFM experiments $[2,5]$ two major differences can be pointed out. Firstly, as for the other experiments performed in the scope of this thesis, there is no solid support during the measurements. The filaments are freely fluctuating between the two beads in the buffer solution. The other difference is that not the whole sample solution is incubated with glutaraldehyde at the same time, but a single filament is first captured between the two trapped beads and then moved into a glutaraldehyde containing buffer solution for five minutes. Importantly, the filaments are stretched to their contour length while the fixation takes place. This means that cross-linking is restricted to amino acids that are structurally closely related, no "loop" formation or fixation of buckling is possible.

To ensure comparability the data of glutaraldehyde stabilized filaments were processed the same way as the data of non-stabilized filaments. In the following sections the data of both, the stabilized and the non-stabilized filaments, are shown next to each other and to the same scale. 


\subsection{Loading-Rate-Dependency}

A
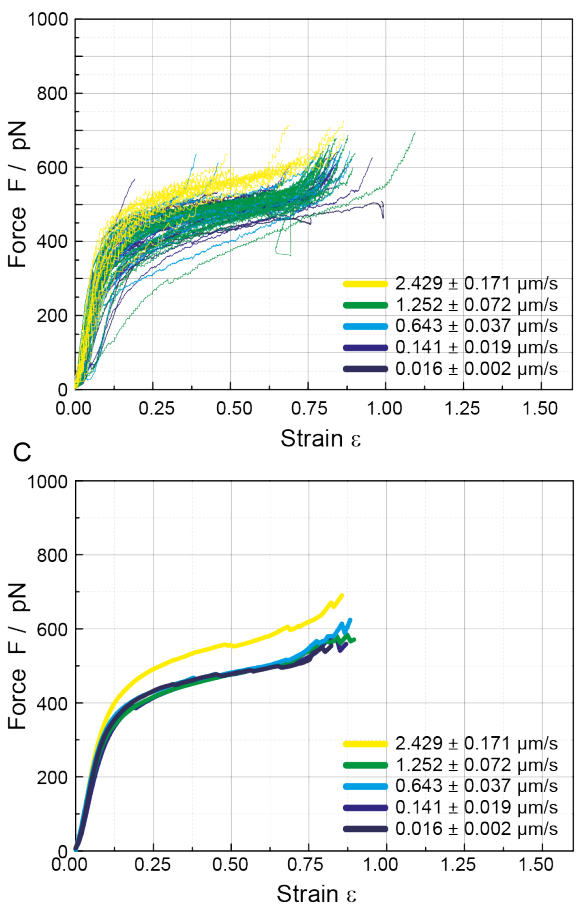

B
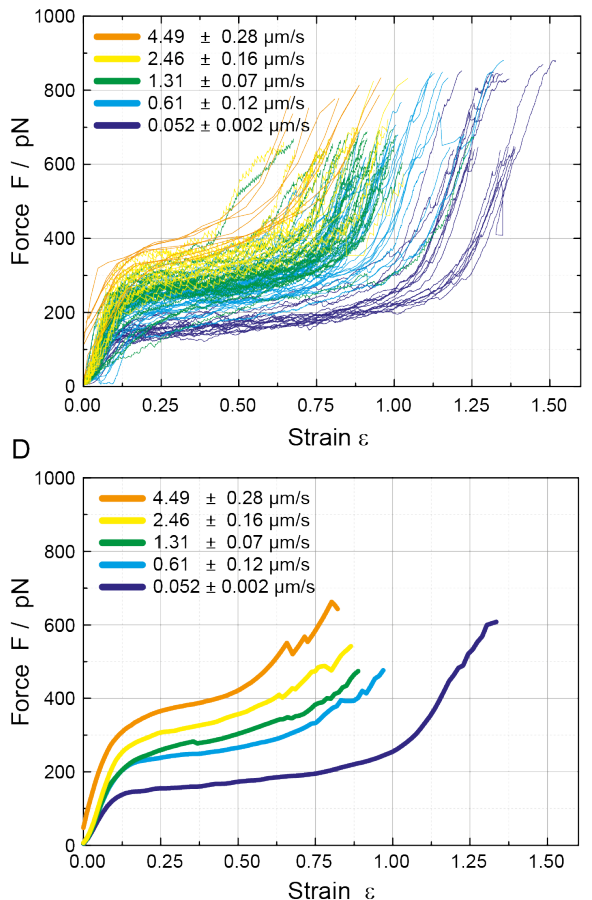

Figure 6.2: Velocity dependent behavior of untreated and glutaraldehyde stabilized vimentin filaments. Filaments stretched with approximately the same loading rate are plotted in the same color for stabilized and non-stabilzed filaments. Individual (A) and average (C) curves of glutaraldehyde stabilized filaments stretched with five different loading rates covering two orders of magnitude (see color code). Individual (B) and average (D) curves of non-stabilized filaments at five different loading rates covering two orders of magnitude (see color code).

Untreated vimentin filaments exhibit a clear loading-rate dependent stretching behavior for loading-rates ranging from 0.05 to $4.5 \mu \mathrm{m} / \mathrm{s}$ [12] as shown in Fig. 6.2 B and D. In comparison, filaments stretched with loading-rates ranging from 0.02 to $2.5 \mu \mathrm{m} / \mathrm{s}$ after glutaraldehyde stabilization, presented in Fig. 6.2 A do not show this clear dependency. Albeit, the average curves per velocity (Fig. 6.2 D) reveal that filaments stretched with the fastest velocity $(2.5 \mu \mathrm{m} / \mathrm{s})$ are shifted towards slightly higher forces compared 
to filaments stretched with velocities ranging from 0.02 to $1.25 \mu \mathrm{m} / \mathrm{s}$. It is therefore not possible to exclude a loading-rate dependent behavior at higher loading-rates. However, compared to untreated vimentin filaments, the loading-rate dependency is at least reduced and the plateau region, where in untreated filaments the $\alpha$-helix to $\beta$ sheet transition takes place, is clearly shifted to higher force values.

Taking the results from references [6] and [8] and the data presented in Fig. 6.2 together it is plausible that the mechanism of $\alpha$-helix unfolding is the basis for filament stretchability also in glutaraldehyde stabilized filaments, though unfolding of several parallel subunits at the same time is most likely required. This also explains the higher plateau force as presumably a higher force is needed to unfold several cross-linked $\alpha$ helices simultaneously than one parallel helix after the other.

Interestingly, more force is needed to stretch glutaraldehyde stabilized filaments but the final strain is comparable to untreated filaments that were stretched at medium to high loading rates. According to the two-state model [12], the reduced final strain in untreated filaments can be attributed to $\alpha$-helices or parts of $\alpha$-helices that do not unfold anymore. Looking into the cross-links Steinert et al. found for vimentin, it might be possible that an unfolding of parts of the monomers is not possible anymore or that they are so tightly cross-linked that the amount of force applied by an OT is not sufficient to unfold this parts.

Only four of the suggested cross-linking positions are directly related to the paststutter-part of the vimentin coil 2, two of those are at the very end of coil 2 and two lie within the coil. The other 12 cross-links are within or at both ends of the coil 1 region, leading to the hypothesis that coil 2 can still unfold more easily than the other parts of the monomer. This well fits the unfolding behavior of untreated filaments at higher loading rates, as also here the coil 2 does most likely unfold first and coil 1A and $1 \mathrm{~B}$ are less likely to open up.

Fig. 6.3 shows examples of full stretching-relaxation cycles of glutaraldehyde stabilized (Fig. 6.3 A) and untreated (Fig. 6.3 B) vimentin IFs. The full reversibility of the strain in stabilized filaments further supports the hypothesis that the basic stretching mechanism in stabilized filaments is the same as in untreated filaments. However, the relaxation curve possesses a slightly different shape indicating a faster refolding at higher force values. This altered relaxation behavior compared to untreated filaments 
A

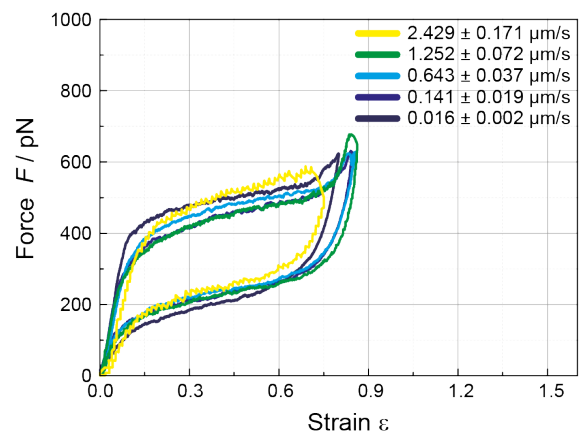

B

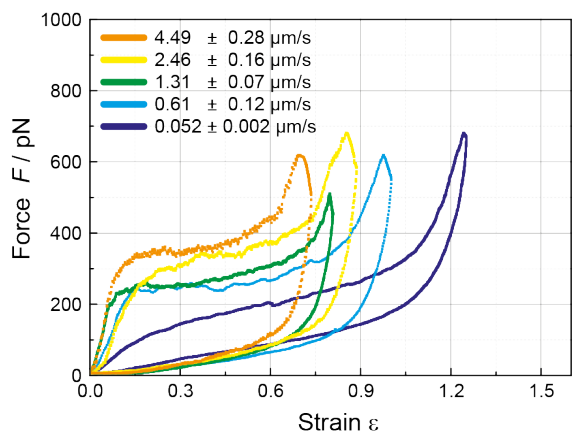

Figure 6.3: Single force-strain cycles of individual vimentin filaments at five different loading rates (see color code). (A) Full cycles of glutaraldehyde stabilized filaments - one example curve per velocity. (B) Full cycles of untreated filaments - one example curve per velocity.

has an additional effect. The energy dissipated during a full stretching-relaxation cycle in stabilized filaments is reduced by about half compared to untreated vimentin filaments. Fig. 6.4 shows a histogram of the dissipated energy for all filaments as percent of the energy that is introduced in the system due to the applied force. Further analysis of the dissipated energy, e.g. in matters of loading-rate and maximum reached strain can be found in Appendix A.

\subsection{Creep Behavior at Constant Force}

Comparing constant force experiments of untreated (Fig. 6.5 B and D) and stabilized (Fig. 6.5 A and C) vimentin filaments reveals several effects of the glutaraldehyde treatment. The most prominent one is again the "unextensibility" at small forces. While FCs at $100 \mathrm{pN}$ in untreated filaments on average yield a strain of 0.4 and the filaments are stable only for some minutes to half an hour, the tested stabilized filament yielded a final strain of less than 0.03 after one hour of applied force. The effect is similar comparing the FCs at $250 \mathrm{pN}$ of both filament types. After one hour of constant pulling stabilized filaments yield a strain of less than 0.1 , while untreated filaments reached a strain of more than 1 in the same time. However, considering the FCs of glutaraldehyde stabilized filaments at $500 \mathrm{pN}$, there is a clear increase in filament extension which can 


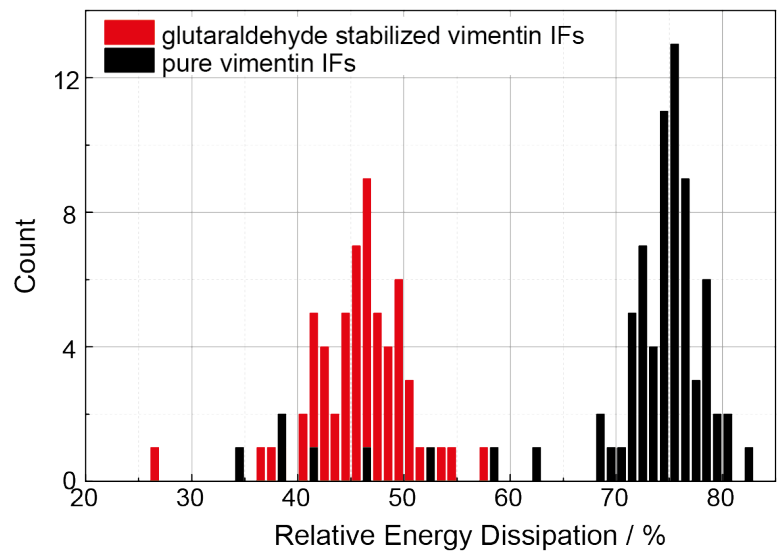

Figure 6.4: Relative energy dissipation in glutaraldehyde stabilized and untreated filaments.

be explained by the fact that the plateau, where the $\alpha$ - $\beta$-transition is presumed to happen, starts at approximately that force in stabilized filaments (Fig. 6.2 A). As the plateau in untreated filaments is reached at about $250 \mathrm{pN}$ one should compare the $250 \mathrm{pN} \mathrm{FC}$ measurements of untreated and the $500 \mathrm{pN}$ FC experiments of stabilized vimentin. When stretched by a force that overcomes the plateau force, the final strain in stabilized filaments levels of at about 0.8 while in untreated vimentin a strain of about 1.2 to 1.4 is reached.

The step size distribution of stabilized filaments under constant load is shown in Fig. 6.6 in individual graphs per force. Again, the step sizes and the percentage steps contribute to the elongation in stabilized filaments is similar compared to untreated vimentin filaments.

\subsection{Discussion}

Even though the glutaraldehyde reaction is known to be reversible [1], the one and two hour force clamp measurements give evidence that this reversibility does not play a role under the given experimental conditions. Especially the 100 and $250 \mathrm{pN} \mathrm{FC} \mathrm{data}$ do not show any effect that would indicate that glutaraldehyde is "washed out". The 
A

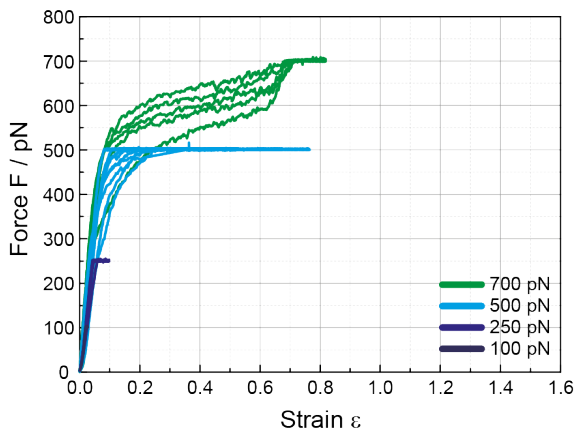

C

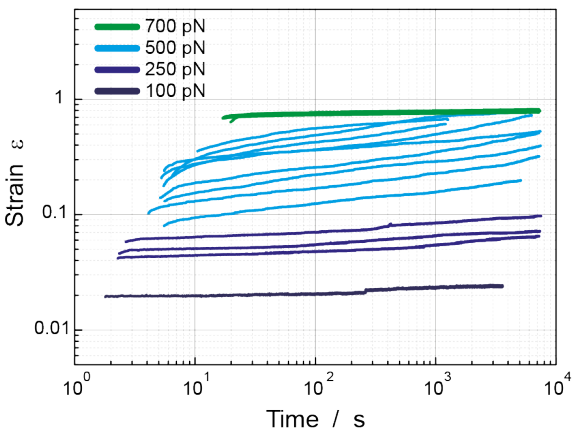

B

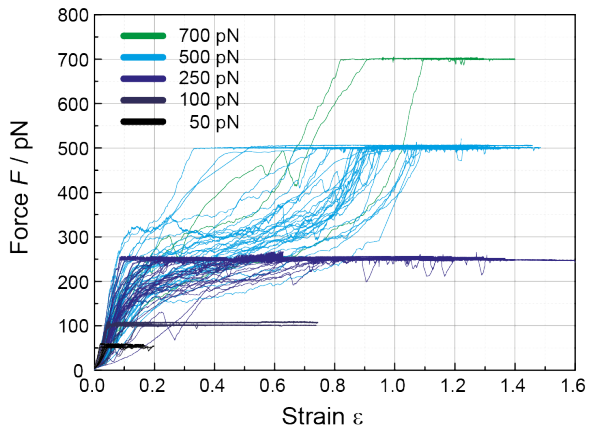

$\mathrm{D}$

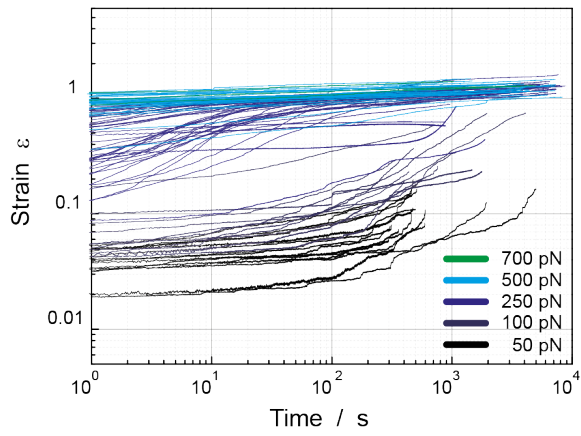

Figure 6.5: Constant force experiments of glutaraldehyde stabilized and non-stabilized vimentin IFs presented in force-strain plots and strain-time plots in a double logarithmic scale. (A,C) About 20 individual glutaraldehyde stabilized filaments stretched at constant forces ranging from 100 to $700 \mathrm{pN}$ (see color code). (B,D) About 100 individual untreated filaments stretched at constant forces ranging from 50 to $700 \mathrm{pN}$ (see color code).

measurements are stable and the filaments' response does not change over time.

An effect due to glutaraldehyde stabilization is clearly visible, especially at forces below $500 \mathrm{pN}$. However, it is not straight forward to reveal how the molecular structure is modified or rather which part of the transition or sliding is altered. Comparing all experiments it seems to be clear that the stabilization with glutaraldehyde did not freeze one of the mechanisms exclusively but altered the mechanical response bearing on the force level where the plateau is reached and on the maximum strain that can be reached by this setup. 

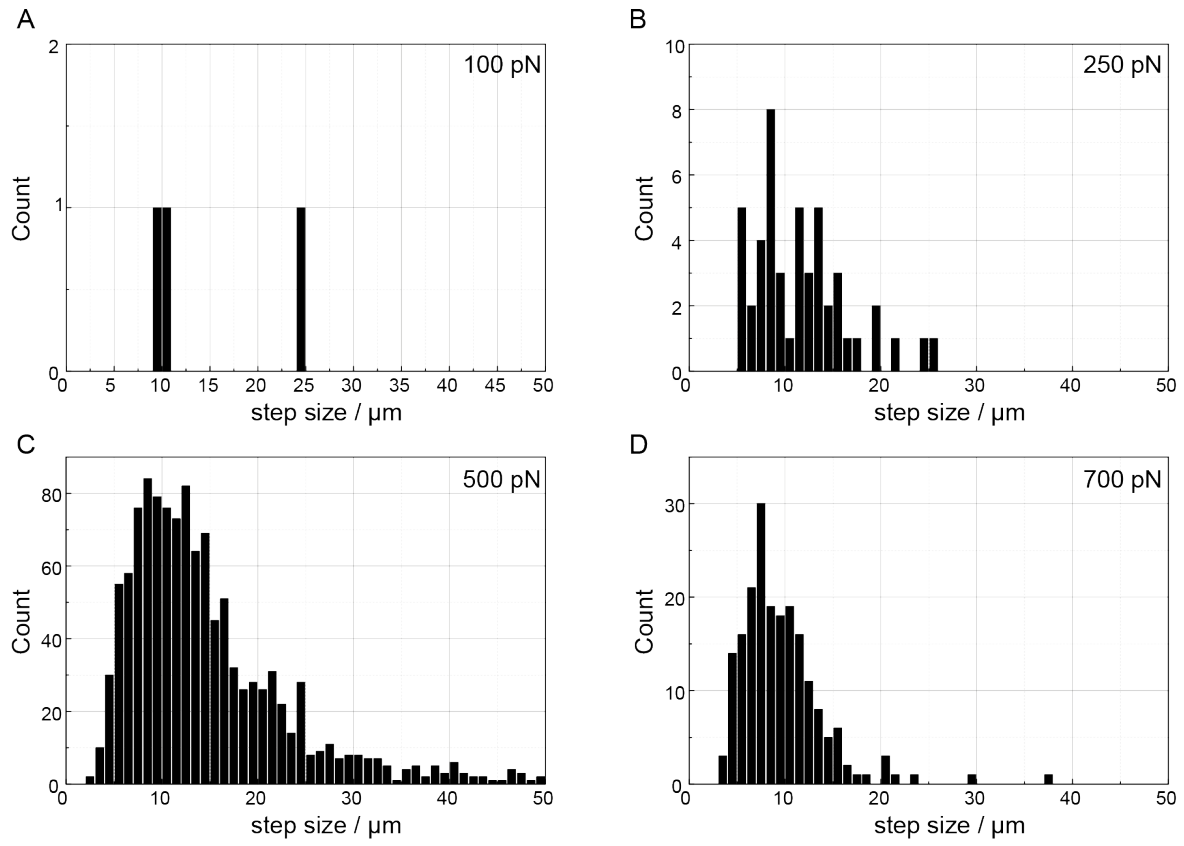

Figure 6.6: Step size histograms of glutaraldehyde stabilized vimentin filaments. (A) Data from one filament at $100 \mathrm{pN}$. (B) Data from three filaments at $250 \mathrm{pN}$. (C) Data from nine filaments at $500 \mathrm{pN}$. (D) Data from five filaments at $700 \mathrm{pN}$.

One explanation for the altered mechanical response of glutaraldehyde treated filaments would be that parallel $\alpha$-helices are cross-linked and require a simultaneous unfolding. This presumable leads to a higher unfolding force as observed in the experiments refering to the shifted force plateau and the reduced strain reached by FCs below $500 \mathrm{pN}$. Tight cross-linking of parts of the filaments' monomers could explain why the final strain is reduced compared to fully unfolded untreated filaments. The comparable strain for glutaraldehyde stabilized filaments and untreated filaments at medium to high loading rates also fits to this hypothesis as many of the cross-linking positions were found in the region of coil 1A and 1B [8] and these are also the parts that are more unlikely to unfold in untreated filaments at higher loading rates [12]. 


\section{References}

1. Migneault, I., Dartiguenave, C., Bertrand, M. J. \& Waldron, K. C. Glutaraldehyde: Behavior in Aqueous Solution, Reaction with Proteins, and Application to Enzyme Crosslinking. BioTechniques 37, 790-802 (2004).

2. Guzmán, C. et al. Exploring the Mechanical Properties of Single Vimentin Intermediate Filaments by Atomic Force Microscopy. J. Mol. Biol. 360, 623-630 (2006).

3. Herrmann, H. et al. Structure and Assembly Properties of the Intermediate Filament Protein Vimentin: The Role of Its Head, Rod and Tail Domains. J. Mol. Biol. 264, 933-953 (1996).

4. Ando, S. et al. Morphological Analysis of Glutaraldehyde-Fixed Vimentin Intermediate Filaments and Assembly-Intermediates by Atomic Force Microscopy. Biochim. Biophys. Acta 1702, 53-65 (2004).

5. Mücke, N., Kirmse, R., Wedig, T., Leterrier, J. F. \& Kreplak, L. Investigation of the Morphology of Intermediate Filaments Adsorbed to Different Solid Supports. J. Struct. Biol. 150, 268-276 (2005).

6. Steinert, P. M., Marekov, L. N. \& Parry, D. A. Diversity of Intermediate Filament Structure. Evidence that the Alignment of Coiled-Coil Molecules in Vimentin is Different from that in Keratin Intermediate Filaments. J. Biol. Chem. 268, 2491624925 (1993).

7. Block, J., Schroeder, V., Pawelzyk, P., Willenbacher, N. \& Koster, S. Physical Properties of Cytoplasmic Intermediate Filaments. Biochim. Biophys. Acta 1853, 30533064 (2015).

8. Downing, D. T. Chemical Cross-Linking Between Lysine Groups in Vimentin Oligomers is Dependent on Local Peptide Conformations. Proteins 25, 215-224 (1996).

9. Fudge, D. S., Gardner, K. H., Forsyth, V. T., Riekel, C. \& Gosline, J. M. The Mechanical Properties of Hydrated Intermediate Filaments: Insights from Hagfish Slime Threads. Biophys J 85, 2015-2027 (2003).

10. Qin, Z., Kreplak, L. \& Buehler, M. J. Hierarchical Structure Controls Nanomechanical Properties of Vimentin Intermediate Filaments. PLOS One 4, e7294 (2009). 
11. Bendit, E. G. A Quantitative X-Ray Diffraction Study of the Alpha-Beta Transformation in Wool Keratin. Text. Res. J. 30, 547-555 (1960).

12. Block, J. et al. Nonlinear Loading-Rate-Dependent Force Response of Individual Vimentin Intermediate Filaments to Applied Strain. Phys. Rev. Lett. 118, 048101 (2017). 



\section{Chapter 7}

\section{Summary, Discussion and}

\section{Conclusion}

Single vimentin filaments show the remarkable physical properties of IFs that were already presumed from mechanical measurements of IF networks and fibers. Here, based on the literature, two theoretical models, taking different levels of the architecture of mature vimentin filaments into account, were used to fit and model the collected data. The elastically coupled two-state model, adapted from references [1, 2], assumes that the parallel monomers in each ULF can be modeled as one effective element [3] yielding a system of entropic springs connected to an elastic module [4]. In this model it is taken into account that the vimentin monomer contains three $\alpha$-helical sections that are connected by linker regions. To incorporate the observed viscous behavior, a dashpot is introduced in the two-state model as a third element, additionally to the elastic and the entropic springs. The second, stochastic, model takes the parallel elements into account yielding a better description of the parallel elements in the ULF architecture. However in this model viscous and entropic contributions to the mechanics of vimentin are neglected and the architecture of the monomer containing three $\alpha$-helical parts is not explicitly taken into account. The filament is described as a chain of ULFs and each ULF contains one spring describing the linker regions as well as the ULF to ULF connection, and 32 monomers described by their spring constant and an 
element that can switch between the shorter $\alpha$-state and the elongated $\beta$-state. Being aware that both models are restricted concerning their application (described in Section 5.4) it was possible to relate the molecular structure of vimentin to the observed mechanical response.

The three different regimes that can be discerned in stretching curves of single vimentin IFs [4] can be attributed to an elastic response of the stretched $\alpha$-helices [5-7], the transition of $\alpha$-helices into $\beta$-sheets [5, 8, 9] and pulling on the beta-sheets [5], respectively. Even though it was not possible to directly prove the $\alpha$-helix to $\beta$-sheet transition in vimentin in the scope of this thesis, the well fitting theory (Chapters 4 , and 5) as well as computer simulations [5] and the direct observation of this mechanism in vimentin hydrogels [9] support this hypothesis. Due to the loading-rate dependent behavior [4], vimentin is very soft when pulled slowly, but much stiffer in consequence of a sudden, fast load. On the molecular level this behavior can be described when the three $\alpha$-helices that form the monomers possess different probabilities to unfold and these probabilities are loading-rate dependent $[4,10]$.

By comparing the stretching and the retraction curve of single vimentin IFs that were stretched to about $600 \mathrm{pN}$ and subsequently relaxed, a pronounced hysteresis becomes evident. Whether the energy uptake by the filament that is equal to the area between the stretching and the relaxation curve in the force over strain graph is directly dissipated or stored in the changed conformation of the filament is not easily distinguishable. Fitting the two-state model [4] to the data indicates that the mechanism for the apparent energy dissipation is mainly the non-equilibrium $\alpha$-helix to $\beta$-sheet transition and only to a minor extend due to viscous contributions (Section 5.3.1).

An additional indicator for different modes of filament elongation was found by constant force experiments. A qualitatively different elongation behavior was observed in filaments pulled at constant forces below or in the force regime of the plateau compared to filaments pulled at constant forces above the plateau force. While under low constant forces filaments mainly elongate by discrete steps, the elongation under high forces is dominated by creep (Section 5.3.3). Step detection revealed step sizes that correspond well to the transformation of $\alpha$-helices into $\beta$-sheets (Section 5.3.3 and Fig. $5.3 \mathrm{D}$ ) whereas the creep behavior could be explained by sliding of subunits against each other as suggested by simulations of the vimentin tetramer [5]. 
Repeated stretching-relaxation cycles, where filaments were pulled to increasing distances, revealed a complex dependency of the filaments' mechanics on the strain history. While the filaments soften with each cycle, which is indicated by the decrease of the initial slope of each stretching event, they reach their initial length upon each relaxation (Section 5.3.2). The mechanism for this behavior could be encoded in the parallel subunits within a filament. Assuming one ULF opens up after the other and the length of a ULF is determined only by its shortest member, elongation of the filament would be based on the full $\alpha$-helix to $\beta$-sheet transition in ULFs. Upon force release the $\alpha$-helices start to rebuild and when at least one of the parallel monomers in each ULF has changed back to its $\alpha$-helical state, the filament reaches its initial length (Section 5.3.2).

The idea to restrict parts of the mechanical properties of vimentin by glutaraldehyde stabilization of the filaments prior to the stretching experiment did not yield exclusive observation of one of the hypothesized mechanisms. In fact it seems to restrict parts of both mechanisms, the $\alpha$ - $\beta$-transition as well as sliding of subunits as the strain that can be reached by OT measurements is decreased but the observed basic mechanisms are the same in stabilized and untreated filaments. The differences in force and strain levels between stabilized and untreated filaments may be explained by cross-linking of parallel helices and the requirement of simultaneous unfolding. Similarities between stabilized and untreated filaments stretched at higher loading rates may be explained based on literature that predicts more cross-linking in vimentin coil $1 \mathrm{~A}$ and $1 \mathrm{~B}$ than in coil $2[11,12]$ and the finding that loading-rate dependency may be originated in the higher probability of vimentin coil 2 to unfold [4].

How exactly the physical properties of vimentin IFs found in the scope of this thesis contribute to the physical properties of cells, tissue and organisms is not straight forward to interpret. The step from single filaments to cells or even organisms is also the step from single filaments to a vimentin network that is incorporated in a complex, interactive system. Pure vimentin networks in vitro show a similar behavior due to strain as seen in single filament experiments. While the network is soft at small strains, it hardens at larger strain [13]. Revealing the functions of a single protein and how it influences the others is not always straight forward and the cooperation of different proteins not necessarily exclusive. Vimentin was found to fulfill several roles 
and interact with a plethora of other proteins. Disassembly of the vimentin network in 3T3 fibroblasts by microinjection of vimentin 1A peptide (a 35 amino acids long vimentin peptide) dramatically changes the cell shape and has a destabilizing effect on the other cytoskeletal filaments and the adhesiveness of the cells [14]. As mentioned above, Brown et al. hypothesize that vimentin is important to control the deformability of T-lymphocyte [15]. Additionally vimentin was found to be important for the mechanical integrity of cells [16, 17] and the localization of cell components [16].

However mice with a vimentin null mutation in their germ line were found to possess a normal phenotype, develop and reproduce, meaning that a complete deletion of vimentin is not generally lethal under non-pathological conditions [18]. In pathological situations it was found that wound healing in vimentin null mice is slower than in wild type mice especially concerning the invasion of fibroblasts and the contraction of the connective tissue [19]. The ablation of three quarters of the kidney mass was lethal by kidney failure for vimentin null but not for wild type mice [20]. In this concern vimentin might be important to modulate the vascular tone as reduction of kidney mass leads to vasodilation in the kideny in non-mutated mice. Lethality was $100 \%$ prevented by application of bosentan an endothelin antagonist [20]. A related finding is that vimentin expression is elevated in vascular cells that are exposed to higher stress levels like the blood pressure in the pulmonary trunk or the left ventricle [21].

Based on the literature findings reviewed above and in Section 2.2 there are reasonable arguments to speculate about the relation between the mechanical properties of single vimentin filaments and the role of vimentin in cells and tissue. One should keep in mind that all processes in the cell are highly complex and especially the kidney experiment [20] reveals that mechanical properties, structural changes and the biochemistry of signalling are highly interconnected. Regarding vimentin and the vascular tone one could speculate that the structural changes in the filaments due to applied strain also enable other binding partners to bind and therefore trigger other protein pathways leading to an adaption to e.g. vasolidation or higher blood pressure of the whole cell.

Already in 1997 Galou et al. summarized that tissue lacking its normal IFs is less stable and cannot resist physical stresses as well as tissue without the IF knockout [22]. The tensile memory of the vimentin filaments may lead to endothelial cells that are very well adapted, e.g., to the regular pressure shift in the vascular system due to the 
normal beating of the heart. The hypothesized structural mechanism of incomplete $\beta$-sheet to $\alpha$-helix re-transition may provide two further advantages. The first one is that filaments reach their initial length after each stretching event and therefore may prevent enlarging of the cells and the tissue. The second advantage appears at larger strains, when exposed to unusual high stresses vimentin filaments, even though prestreched for several times, may still protect the cells integrity due to the full transformation of $\alpha$-helices into $\beta$-sheets and subsequent stiffening.

For migrating cell types, e.g. fibroblasts, the loading-rate dependency of vimentin means that the IF network is mechanically almost invisible at low velocities like cell migration but may protect the cells when deformed very fast or to unusually large strains. In situations of extreme extension vimentin may also serve as a "shock absorber" dissipating or storing large amounts of energy by $\alpha$-helix to $\beta$-sheet transition.

To conclude, the data collected in the scope of this thesis supports the hypothesis that IFs provide mechanical strength to cells and tissue and suggests an explanation why the importance of IFs becomes visible especially in pathological situations or situations of unusual mechanical load. Being aware that the models used are both limited concerning their application (described in Section 5.4), it was shown that they are consistent with each other and allow to relate the experimentally observed mechanical properties of vimentin to the underlying molecular processes. The transition of $\alpha$ helices into $\beta$-sheets enables vimentin to absorb or dissipate large amounts of energy and forms the basis for a mechanism of tensile memory that allows vimentin to be compliant with repeated stretching at small deformations and resistant against large deformations. Comparing vimentin to other filamentous structures it was found to have both, the elasticity and energy absorbing/dissipating capacity of a transformable $\alpha$-helix at lower strain and force values as well as the resilience and stiffness of materials like spider silk, probably due to the formation of $\beta$-sheets, when stretched to larger strains.

IFs are expressed in a tissue and cell-type dependent manner and are believed to support them with different mechanical properties. Based on the results collected in the scope of this thesis it would now be interesting to expand the experiments on single vimentin filaments to other IF types and compare their mechanical properties to each other and also to the possible requirements of the cell types they are expressed 
in. Another application would be to test how the mechanical response of mutated IFs is changed compared to their wild type as mutations in IFs are known to cause several diseases. 


\section{References}

1. Burte, H. \& Halsey, G. A New Theory of Non-Linear Viscous Elasticity. Text. Res. J. 17, 465-476 (1947).

2. Rief, M. Elastically Coupled Two-Level Systems as a Model for Biopolymer Extensibility. Phys. Rev. Lett. 81, 4764-4767 (1998).

3. Friddle, R. W., Noy, A. \& de Yoreo, J. J. Interpreting the Widespread Nonlinear Force Spectra of Intermolecular Bonds. P. Natl. Acad. Sci. USA 109, 13573-13578 (2012).

4. Block, J. et al. Nonlinear Loading-Rate-Dependent Force Response of Individual Vimentin Intermediate Filaments to Applied Strain. Phys. Rev. Lett. 118, 048101 (2017).

5. Qin, Z., Kreplak, L. \& Buehler, M. J. Hierarchical Structure Controls Nanomechanical Properties of Vimentin Intermediate Filaments. PLOS One 4, e7294 (2009).

6. Qin, Z. \& Buehler, M. J. Structure and Dynamics of Human Vimentin Intermediate Filament Dimer and Tetramer in Explicit and Implicit Solvent Models. J. Mol. Model. 17, 37-48 (2011).

7. Ackbarow, T. \& Buehler, M. J. Alpha-Helical Protein Domains Unify Strength and Robustness Through Hierarchical Nanostructures. Nanotechnology 20, 075103 (2009).

8. Bendit, E. G. A Quantitative X-Ray Diffraction Study of the Alpha-Beta Transformation in Wool Keratin. Text. Res. J. 30, 547-555 (1960).

9. Pinto, N. et al. Self-Assembly Enhances the Strength of Fibers Made from Vimentin Intermediate Filament Proteins. Biomacromolecules 15, 574-581 (2014).

10. Bell, G. Models for the Specific Adhesion of Cells to Cells. Science 200, 618-627 (1978).

11. Steinert, P. M., Marekov, L. N. \& Parry, D. A. Diversity of Intermediate Filament Structure. Evidence that the Alignment of Coiled-Coil Molecules in Vimentin is Different from that in Keratin Intermediate Filaments. J. Biol. Chem. 268, 2491624925 (1993). 
12. Downing, D. T. Chemical Cross-Linking Between Lysine Groups in Vimentin Oligomers is Dependent on Local Peptide Conformations. Proteins 25, 215-224 (1996).

13. Janmey, P. A., Euteneuer, U., Traub, P. \& Schliwa, M. Viscoelastic Properties of Vimentin Compared with Other Filamentous Biopolymer Networks. J. Cell Biol. 113, 155-160 (1991).

14. Goldman, R. D., Khuon, S., Chou, Y. H., Opal, P. \& Steinert, P. M. The Function of Intermediate Filaments in Cell Shape and Cytoskeletal Integrity. J. Cell Biol. 134, 971-983 (1996).

15. Brown, M. J., Hallam, J. A., Colucci-Guyon, E. \& Shaw, S. Rigidity of Circulating Lymphocytes Is Primarily Conferred by Vimentin Intermediate Filaments. J. Immunol. 166, 6640-6646 (2001).

16. Guo, M. et al. The Role of Vimentin Intermediate Filaments in Cortical and Cytoplasmic Mechanics. Biophys J 105, 1562-1568 (2013).

17. Mendez, M. G., Restle, D. \& Janmey, P. A. Vimentin Enhances Cell Elastic Behavior and Protects Against Compressive Stress. Biophys J 107, 314-323 (2014).

18. Colucci-Guyon, E. et al. Mice lacking Vimentin Develop and Reproduce without an Obvious Phenotype. 79, 679-694 (1994).

19. Eckes, B. et al. Impaired Wound Healing in Embryonic and Adult Mice Lacking Vimentin. J. Cell Sci. 113 ( Pt 13), 2455-2462 (2000).

20. Terzi, F. et al. Reduction of Renal Mass is Lethal in Mice Lacking Vimentin. Role of Endothelin-Nitric Oxide Imbalance. J. Clin. Invest. 100, 1520-1528 (1997).

21. Schnittler, H.-J., Schmandra, T. \& Drenckhahn, D. Correlation of Endothelial Vimentin Content with Hemodynamic Parameters. Histochem. Cell Biol. 110, 161167 (1998).

22. Galou, M. et al. The Importance of Intermediate Filaments in the Adaptation of Tissues to Mechanical Stress. Evidence from gene knockout studies. 89, 85-97 (1997). 


\section{List of Publications}

Johanna Block, Hannes Witt, Andrea Candelli, Jordi Cabanas Danes, Erwin J.G.Petermann, Gijs J.L.Wuite, Andreas Janshoff and Sarah Köster, Viscoelastic Properties of Vimentin Originate from Nonequilibrium Conformational Changes, Science Advances, 4, eaat1161, 2018, DOI: 10.1126/sciadv.aat1161

Johanna Block, Hannes Witt, Andrea Candelli, Erwin J. G. Petermann, Gijs J. L. Wuite, Andreas Janshoff and Sarah Köster, Nonlinear Loading-Rate-Dependent Force Response of Individual Vimentin Intermediate Filaments to Applied Strain, Physical Review Letters, 118, 048101, 2017, DOI: 10.1103/PhysRevLett.118.048101

Johanna Block, Viktor Schröder, Paul Pawelzyk, Norbert Willenbacher and Sarah Köster, Physical properties of cytoplasmic intermediate filaments, Biochimica et biophysica acta - Molecular Cell Research, 1853, 11, 3053-3064, 2015, DOI: 10.1016/j.bbamcr.2015.05.009 



\section{Lebenslauf}

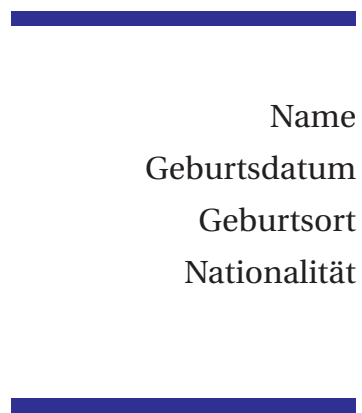

seit 07.2014

$10.2012-06.2014$

\section{Persönliche Daten}

Johanna Lena Block

10.04.1988

Oldenburg (Oldb.)

deutsch

\section{Akademische Ausbildung}

Promotionsstudium im Rahmen der Göttingen Graduate School for Neurosciences, Biophysics, and Molecular Biosciences (GGNB) of the GeorgAugust University School of Science (GAUSS), Göttingen, Germany. Wissenschaftliche Mitarbeiterin am Institut für Röntgenphysik an der Georg-August Universität Göttingen

Master of Science in Biotechnologie, Schwerpunkt: Angewandte Molekularbiologie, Technische Universität Braunschweig 
01.2014 - 06.2014 Masterarbeit: "Cell Stiffness in EMT: A Role for RhoD?", Supervisor: Prof. Dr. Pontus Aspenström, Abteilung für Mikrobiologie, Tumor- und Zellbiologie (MTC), Karolinska Institutet, Stockholm und Prof. Dr. Philip Tinnefeld, Institut für Physikalische Chemie, Technische Universität Braunschweig

$10.2009-09.2012$ Bachelor of Science in Biotechnologie, Schwerpunkt: Bioprozesstechnik, Technische Universität Braunschweig

$06.2012-09.2012$ Bachelorarbeit: "Production, Purification and Fluorescence Labeling of Synthetically Engineered HIV-1 Protease as a Basis for a Single Molecule Drug Screening Assay", Supervisor: Prof. Dr. Philip Tinnefeld, Institut für Physikalische Chemie, Technische Universität Braunschweig

\section{Ausbildung}

$08.2007-09.2009$

Ausbildung zur Pferdewirtin - Schwerpunkt Reiten, Deutsche Reitschule im NordrheinWestfälischen Landgestüt Warendorf

06.2007 Erlangung der allgemeinen Hochschulreife, Jade-Gymnasium, Jaderberg 


\section{Danksagung}

Ich möchte mich bei zahlreichen Menschen bedanken ohne die eine erfolgreiche Anfertigung dieser Arbeit nicht möglich gewesen wäre.

Zuallererst möchte ich Sarah Köster danken, dass sie mir die Möglichkeit gab diese Arbeit in ihrer Gruppe anzufertigen. Die umfassende Betreuung und Förderung, sowie die Möglichkeit meine Arbeit auf zahlreichen nationalen und internationalen Konferenzen vorzustellen, Kontakte zu knüpfen und Diskussionen zu führen hat wesentlich zum Gelingen dieser Arbeit beigetragen. Insbesondere möchte ich mich auch für Sarahs Flexibilität und ihre Unterstützung während meiner Schwangerschaft und auch nach der Geburt meines Sohnes bedanken ohne die ein erfolgreicher Abschluss dieser Arbeit nicht möglich gewesen wäre.

Meine Experimente habe ich zu einem großen Teil in Amsterdam durchgeführt. Mein besonderer Dank gilt Andrea Candelli, der zusammen mit Sarah die Idee für dieses Projekt entwickelt und mich während meiner ersten beiden Messwochen betreut hat. Durch seine Anleitung habe ich schnell ein Gefühl für das Setup bekommen und seine zahlreichen Ideen für weitere Experimente waren stets Inspiration für die Planung meiner Experimente. Auch meinen späteren Betreuern Jordi Cabanas Danes und Margherita Marchetti danke ich herzlich für ihre Hilfe und insbesondere Jordi für die Unterstützung bei der Datenanalyse und dem Einbringen seines chemischen Wissens. Den Leitern der Amsterdamer Arbeitsgruppe Erwin J. G. Petermann und Gijs J. L. Wuite danke ich für die Möglichkeit ihre Labore zu nutzen und für die fachliche Diskussion meiner Daten.

Andreas Janshoff möchte ich für seine Arbeit als Mitglied in meinem Thesis Committee und sein Engagement diese Arbeit als Zweitgutachter zu betreuen danken. Die 
fachlichen Diskussionen und seine Begeisterung für das Thema meiner Dissertation haben meine Arbeit und insbesondere die Auswertung meiner Daten wesentlich vorangetrieben. Dass er seinen Doktoranden Hannes Witt mit ins Boot geholt, war wohl einer der wichtigsten Faktoren dafür, dass ein Großteil der Ergebnisse dieser Dissertation bereits im Rahmen wissenschaftlicher Artikel veröffentlicht wurde oder sich im Prozess der Veröffentlichung befindet. Ich möchte mich bei Hannes Witt für die fachlichen Diskussionen, für das kritische Korrekturlesen meiner Arbeit, für seine Begeisterung für die mechanischen Eigenschaften von Vimentin, aber insbesondere auch für die unglaublich reibungslose und angenehme Zusammenarbeit bedanken.

Florian Rehfeldt danke ich dafür, dass er sich kurzfristig bereiterklärt hat Mitglied meines Thesis Committees zu werden. Die kritische Diskussion meiner Experimente und Daten und sein stets für Fragen offenes Ohr haben mir sehr geholfen.

Bert de Groot, Andreas Egner und Ralf Ficner danke ich dafür, dass sie Mitglieder in meinem Thesis Advisory Bord sind und sich bereiterklärt haben an meiner Verteidigung teilzunehmen.

Für zahlreiche gute Ideen, das Teilen ihres unerschöpflichen Wissens über Intermediärfilamente und die fachlichen Diskussionen möchte ich mich ganz herzlich bei Norbert Müicke und Harald Herrmann bedanken.

Weiter möchte ich mich bei allen aktuellen und früheren Mitgliedern der AG Köster und den Kolleginnen und Kollegen im IRP für die großartige Zusammenheit, die Selbtsverständlichkeit mit der Hilfe angeboten wurde und die gute Atmosphäre in der Gruppe bedanken. Besonders hervorheben möchte ich Rabea Sandmann, die für alle meine Fragen offen war und mir enorm geholfen hat mich schnell in meine Doktorarbeit einzufinden. Von Bernd Nöding habe ich die Grundlagen für meine Experimente und den Umgang mit Vimentin erlernt. Jens Nolting danke ich für seine Hilfe bei der Datenanalyse mit MatLab. Auch Jana Hanke und Manuela Denz möchte ich besonders hervorheben. Beide hatten immer ein offenes Ohr und haben mir mit ausgiebigen Diskussionen über meine Experimente und Ergebnisse und später auch mit kurzen Babysitter-Diensten sehr weitergeholfen. Ich danke Susanne Bauch für die Produktion meines Proteins und für die Unterstützung im Labor.

Jochen Herbst und Jan Goeman danke ich für die stets schnelle und kompetente Lösung aller technischen Fragen und Probleme. Bei den Sekretärinnen den SFB755 Eva 
Hetzel und Christine Wilke-Feist sowie den Sekretärinnen des IRP Kerstin Pluscke und Michaela Ständer möchte ich mich für die Lösung aller bürokratischen Probleme und auch für die Unterstützung beim Finden und Umsetzen von Möglichkeiten zur besseren Vereinbarkeit von Familie und beruflichem Alltag bedanken.

Meine Familie und Freunde sind in allen Zeiten eine unbeschreibliche Stütze. Unter ihnen gilt besonderer Dank Frauke Grabbe und Anika Manzke, die mir durch ihre Unterstützung im (Pferde-)Alltag, insbesondere in Zeiten meiner Abwesenheit und während des Zusammenschreibens dieser Arbeit, eine riesige Erleichertung waren.

Zuletzt möchte ich mich bei meinen Eltern Kathrin und Jürgen, meinen Geschwistern Julia und Lukas, meinem Partner Thomas, seinen Eltern Elisabeth und Helmut und meinem Sohn Jakob bedanken. Ich kann ihnen für die vielen verschiedenen Formen der Unterstützung, mit denen sie zum Gelingen dieser Arbeit beigetragen haben, gar nicht genug danken. 



\section{Appendix A}

\section{Supplementary Information - Energy Dissipation}

Energy dissipation was analyzed in data sets which contain a full stretching and retraction curve. The energy that was introduced into the system by the applied force acting on the filament was calculated from integrating the area enclosed by the stretching curve and the x-axis (blue-striped area in Fig. A.1). The area enclosed by the stretching and the relaxing curve (red area in Fig. A.1) equals the energy that is dissipated when the filament is relaxed. The relative or apparent energy dissipation was calculated from dividing the dissipated energy by the introduced energy.

The amount of energy that was introduced in the system when vimentin filaments were stretched to forces of 550 to $650 \mathrm{pN}$ is comparable in glutaraldehyde stabilized and untreated filaments (Fig. A.2). By comparing the amount of introduced energy sorted by loading-rate, no loading-rate dependent behavior is observed in glutaraldehyde stabilized filaments (Fig. A.2A) while from looking at the histograms the energy that is introduced in untreated filaments seems to slightly increase with increasing loading-rate (Fig. A.2 B). However, when plotting the average values of the introduced and dissipated energy per loading-rate (Fig. A.4) the tendency of the introduced energy to increase with increasing loading-rate seems to be similar in untreated and glutaraldehyde stabilized filaments. To proof whether there is a clear trend it would be 


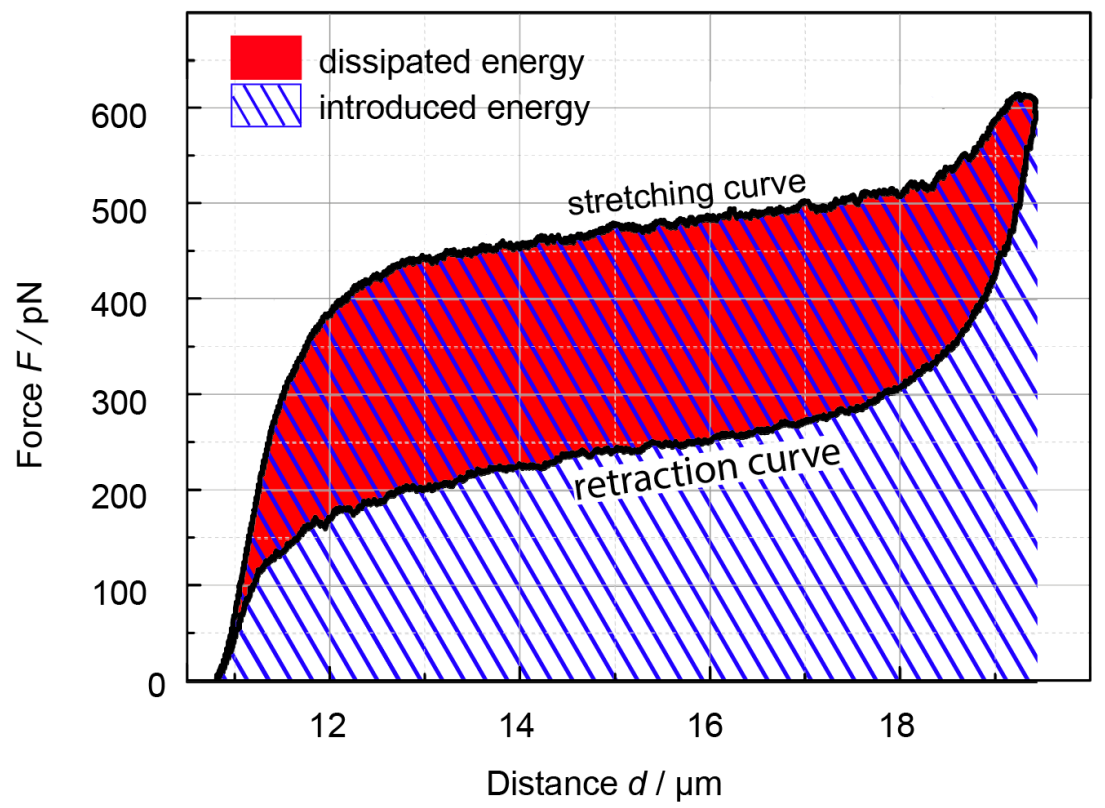

Figure A.1: Schematic representation of areas integrated to calculate energy dissipation from full stretchingrelaxation data sets, exemplarily shown for one data set. The blue striped area represents the energy that was introduced in the system due to an applied force acting on the filament. The red area equals the energy that was dissipated while the filament was relaxed.

necessary to collect more data sets and data sets at different loading rates.

The amount of energy that is dissipated due to the relaxation of the filaments is shown in histograms in Fig. A.3. Comparing again glutaraldehyde stabilized and untreated filaments, the amount of energy that is dissipated in glutaraldehyde stabilized filaments seems to be slightly lower than the energy dissipated due to relaxation in untreated filaments. A loading-rate dependent behavior is not observed in both, the stabilized and the untreated filaments. A small variation is only visible in untreated filaments stretched by the slowest loading-rate, where the amount of dissipated energy is a bit lower.

To ensure that the difference in the relative dissipated energy (Fig. 6.4) is not due to the reduced strain that is reached in glutaraldehyde stabilized filaments (see Fig. 6.3 for comparison), the data was binned by the reached strain. The filaments are binned 
A

glutaraldehyde stabilized

- introduced energy
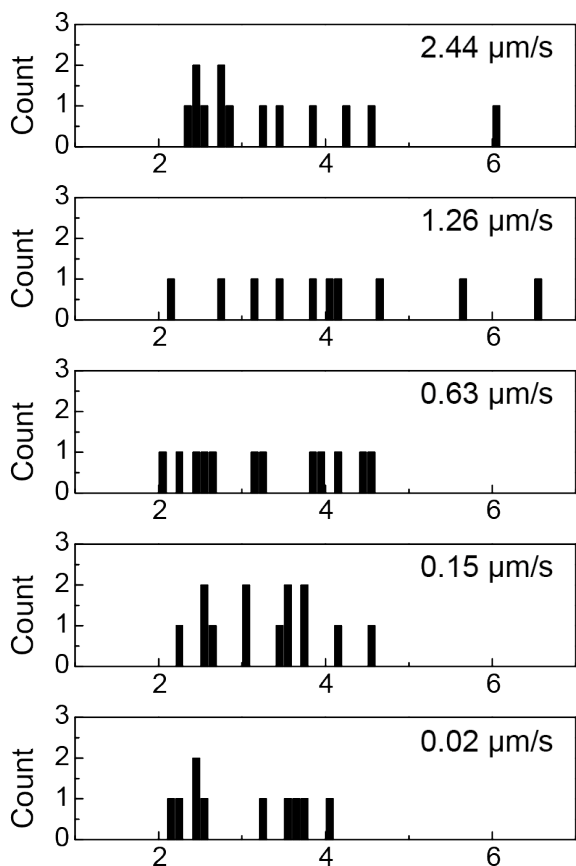

energy $/ 10^{-15} \mathrm{~J}$
B

untreated

- introduced energy
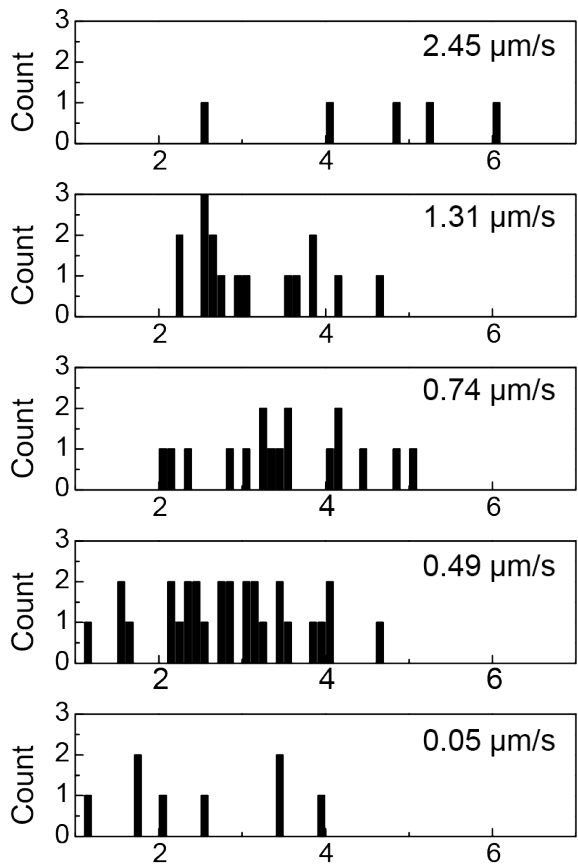

energy $/ 10^{-15} \mathrm{~J}$

Figure A.2: Histograms of introduced energy. (A) glutaraldehyde-stabilized filaments, histograms sorted by loading-rate (B) untreated filaments, histograms sorted by loading-rate

by the maximum strain (from 0.2 to 1.3 ) that was reached in the measurement with a bin size of 0.1. The introduced, the dissipated and the relative energy are plotted in bar charts in Fig. A.5. The reduced strain in glutaraldehyde stabilized filaments is clearly visible also in this representation. However, it becomes also very clear, that the glutaraldehyde stabilized filaments that were stretched to the same strains as pure filaments (0.65 to 0.95 ) show a higher introduced energy (Fig. A.5 A) and a lower energy dissipation (Fig. A.5 B) which finally yields a lower relative energy dissipation in stabilized filaments (Fig. A.5 C). 
A

glutaraldehyde stabilized

- dissipated energy
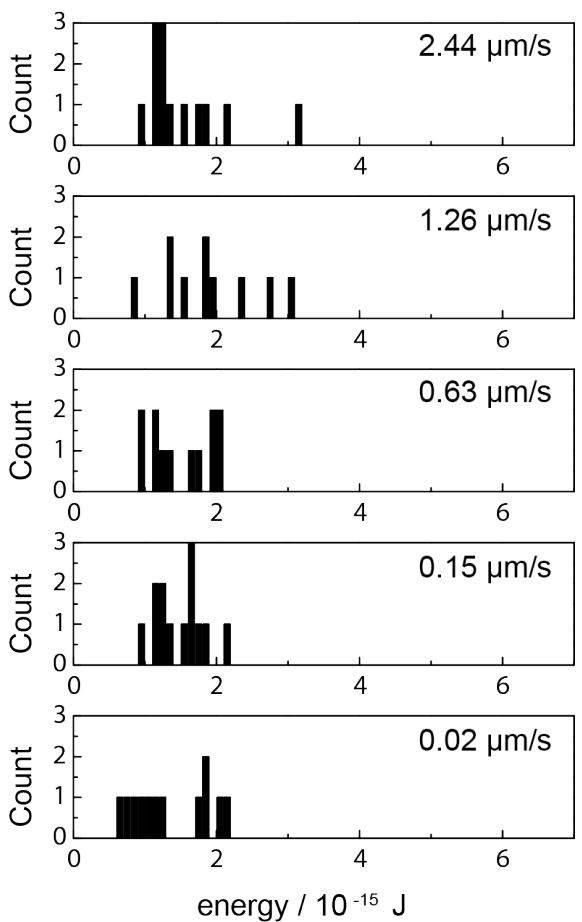

B

untreated

- dissipated energy
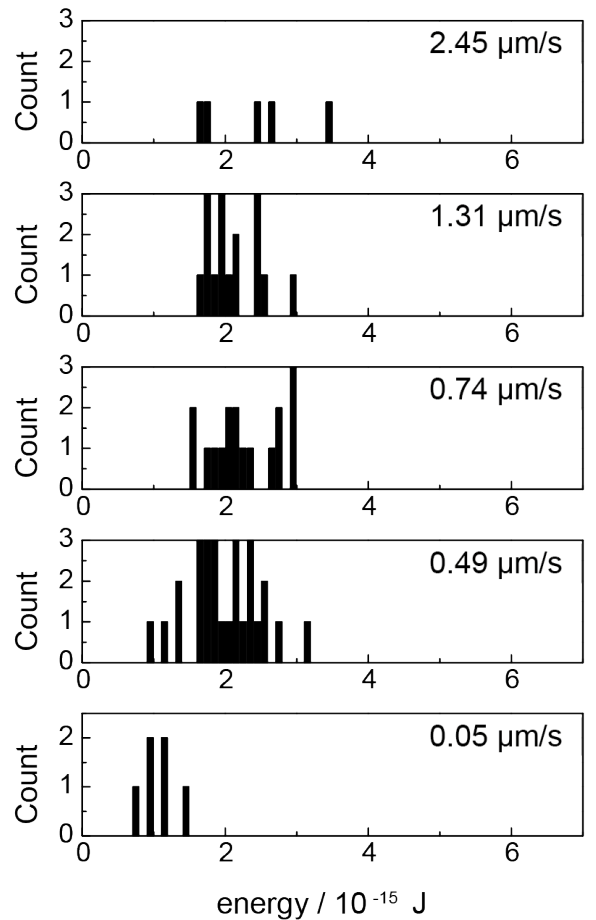

Figure A.3: Histograms of the amount of dissipated energy. Histograms are sorted from bottom to top by increasing loading-rates. (A) glutaraldehyde-stabilized filaments, (B) untreated filaments. 
A

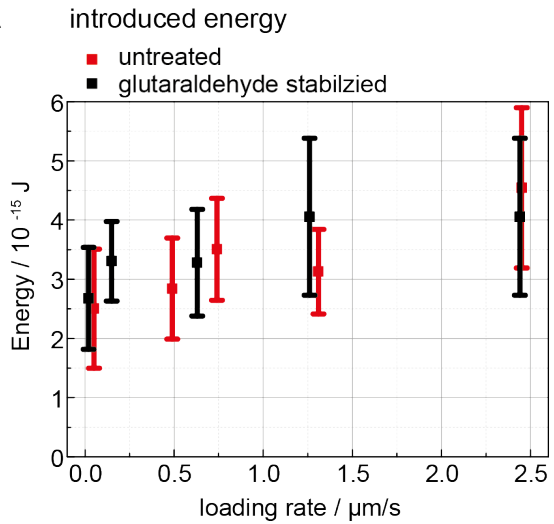

B dissipated energy

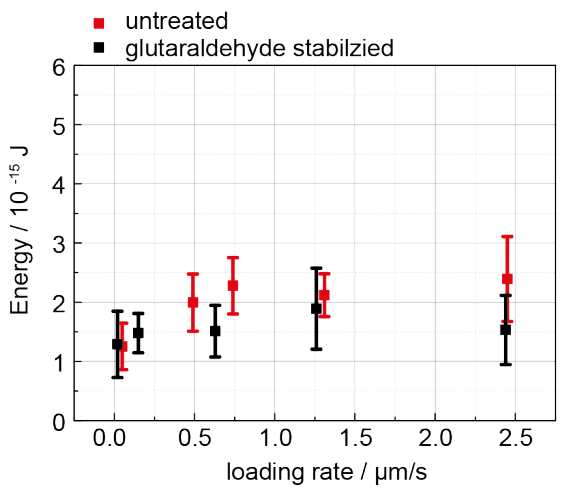

Figure A.4: Average and standard deviation of introduced and dissipated energy for glutaraldehyde stabilized and untreated vimentin filaments sorted by loading-rate. (A) Introduced energy, (B) dissipated energy. 

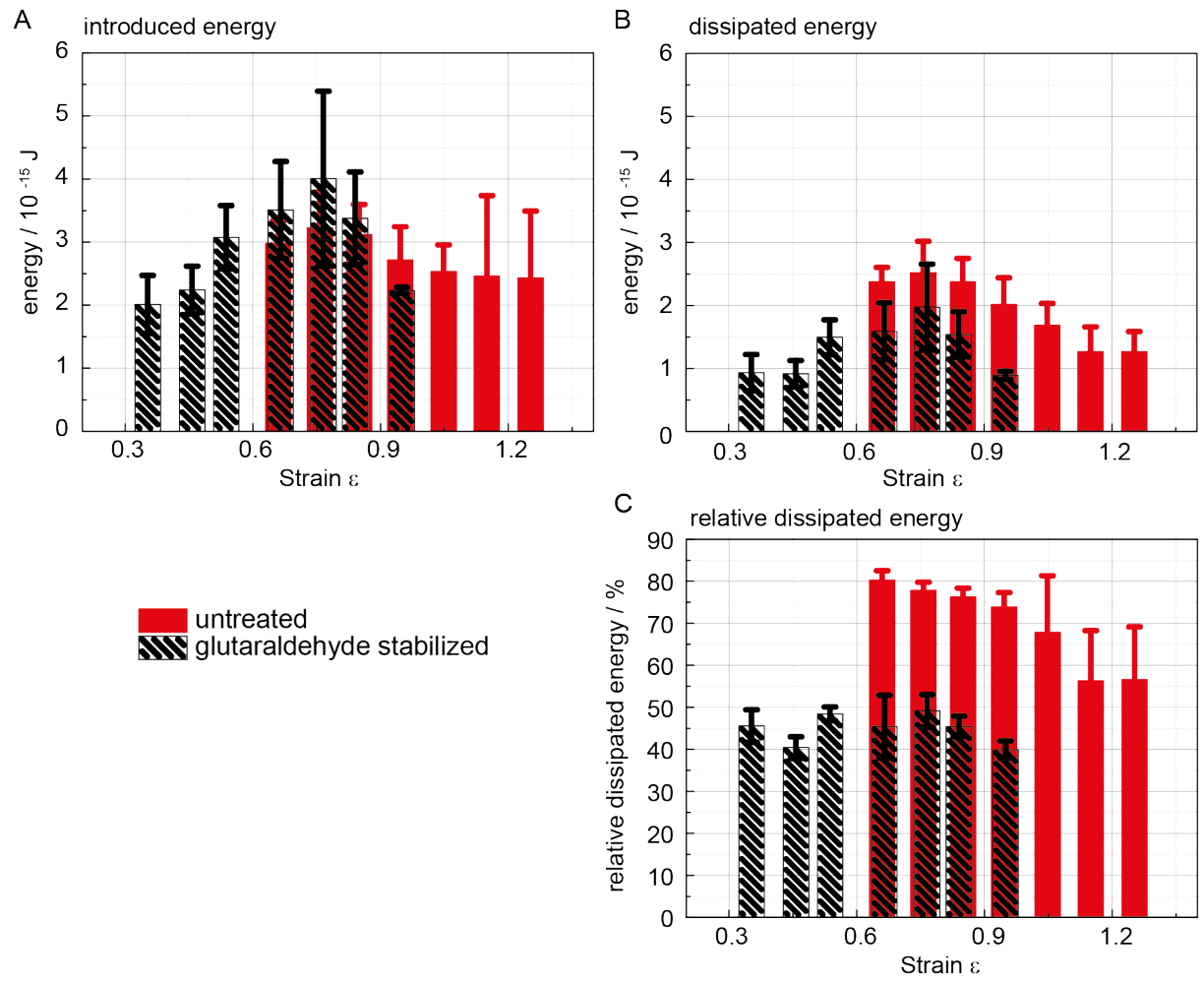

Figure A.5: (A) Introduced, (B) dissipated and $(C)$ relative dissipated energy in glutaraldehyde stabilized and untreated vimentin filaments binned by the maximum reached strain with a bin size of 0.1 . 
C ells are the basic unit of living organisms and consist of a cytoplasm, which is enclosed by a membrane. As building blocks of life with a plethora of functions, cells have to be equipped with a high degree of mechanical resistance, durability, and variability. In eukaryotic cells three filamentous protein types actin filaments, microtubules, and intermediate filaments (IFs) - form the socalled cytoskeleton, a network that is known to play a key role for the mechanical properties of cells. Among the three filament systems, IFs are special in terms of, for example, their hierarchical architecture, and their cell-type specific expression.

In this thesis, vimentin, an IF mostly found in mesenchymal cells, is studied as a model system to learn more about the mechanical properties of IFs, and the underlying mechanisms that determine their robustness, stiffness, and flexibility. Using a combination of optical trapping and atomic force microcopy experiments and stochastic and numerical modelling, vimentin is found to possess impressive physical properties, such as an extendibility of about 3.6 times its initial length and a tensile memory that can be directly linked to the molecular architecture of the protein and the hierarchical construction of the filament.

The experimental results show a clear loading-rate- and strain-dependent behavior of single vimentin IFs supporting the hypothesis that vimentin acts as a "safety belt" for cells, protecting them especially at large and fast deformations. The potential to dissipate a large amount of energy that is attributed to distinct non-equilibrium unfolding and refolding of the $\alpha$-helices, which are the main structural feature of the vimentin monomer, enables vimentin to act as a shock absorber when exposed to large deformations. In case of cyclic deformations, such as in the cardiovascular system, the observed tensile memory could potentially help cells to be compliant with the repeated strain.

In conclusion, vimentin is found to display highly interesting and diverse mechanical properties depending on the applied stress that could be linked to the molecular architecture of the filaments and enable vimentin to determine the mechanical properties of cells to a large extend. 